\title{
Paleontology of leaf beetles
}

\author{
JORGE A. SANTIAGO-BLAY
}

Department of Entomological Sciences, University of California, Berkeley, CA 94720-0001, USA

\begin{abstract}
'The rate of evolution in any large group is not uniform; there are periods of relative stability, and periods of comparatively rapid change.'

Cockerell and LeVeque, 1931
\end{abstract}

To Yenli Yeh, my beloved wife, a most wonderful

person!

\begin{abstract}
The fossil record of the Chrysomelidae can be tentatively traced back to the late Paleozoic to early Mesozoic (Triassic). Mesozoic records (at least 9 subfamilies, 19 genera, and 35 species), are represented by the Sagrinae, the exclusively Mesozoic Protoscelinae, Clytrinae, Cryptocephalinae, Eumolpinae, Chrysomelinae, Galerucinac, Alticinae, and Cassidinae. Cenozoic records (at least 12 subfamilies $-63 \%$ of the extant - 121 genera, and 325 species), include the same extant subfamilies as well as the Donaciinae, Zeugophorinae, Criocerinae, and Hispinae and can be frequently identified to genus, especially if preserved in amber. Quaternary records are often identified to extant species. In total, at least 131 genera (about $4 \%$ of total extant), and 357 species $(<1 \%)$ have been reported. At least, 24 genera $(<1 \%$ of the extant) seem to be extinct.

Although reliable biological information associated with the fossil chrysomelids is very scarce, it seems that most of the modern host-plant associations were established, at least, in the late Mesozoic to early Cenozoic. As a whole, stasis scems to be the general rule of the chrysomelid fossil record. Together with other faunal elements, chrysomelids, especially donaciines, have been used as biogeographic and paleoclimatological indicators in the Holocene.
\end{abstract}

\section{Introduction}

The Chrysomelidae constitute one of the most abundant and diverse families of living organisms. Chrysomelids are known as leaf beetles because, at least, in one stage of their life history they feed on ljving plant tissues such as leaves or roots. With more than 50,000 extant, described species (Lopatin, 1984) distributed in 19 subfamilies (Seeno \& Wilcox, 1982; Suzuki, 1985), this family probably ranks among the top 20 of living forms in number of species (Strong et al., 1984). Based on Erwin's (1983) estimates, the number of described species will undoubtedly grow, at least, by a factor of three, although precise extrapolations are difficult (May, 1990).

Despite the abundance and awareness of leaf beetles in the present, their fossil history is little known. I am not aware of review papers on the fossil record of this family as a whole. Goecke (1943, 1960a) and Jolivet (1970) treated the fossil Donaciinae. A significant number of species descriptions date from the second half of the 1800's (e.g. Heer or Scudder, and others, in References) or treat the subject in connection with larger topics, such as the evolution of the Insecta (Carpenter, 1930, 1992; Larsson, 1978) or the Coleoptera (Crowson, 1975, 1981). This, as well as other insect families, are overlooked in a well-known invertebrate paleontology book (Moore et al., 1952). Even though chrysomelids are relatively abundant in the fossil record (Ander, 1942; Villiers, 1979), many authors have noted that most specimens in collections remain undetermined (Crowson, 1981; Larsson, 1978; Lutz, 1990) probably because most specimens are known only from elytra or parts of elytra (Brodie, 1845; Debray, 1873; Martynova, 1961; Westwood, 1854).

This chapter attempts to compile our knowledge of the fossil leaf beetles and, I hope, it will serve as a supplement to the extraordinary annotated generic list of extant chrysomelids by Seeno and Wilcox (1982). This compilation is a first step towards a meaningful use of fossils integratively to assess the phylogeny of any group (Jablonski et al., 1985). This paper is not a taxonomic revision. Since 1986, and in collaboration with other researchers, I have been working on fossil chrysomelids, particularly but not exclusively, those preserved in amber. The detailed results of those investigations will soon be submitted for publication elsewhere. Since our knowledge of fossil chrysomelids must still be regarded as incomplete and to save space, I have decided not to include keys to and diagnoses of the genera of fossil chrysomelids. The reader is referred to the tables and the figures, as well as to the specialized, pertinent references. 


\section{A systematist's pandemonium}

The effort to compile the data herein included faced many challenges. The task of sorting through the enormous, largely uncatalogued, and diversely languaged paleozoological literature proved to be huge. Paleoentomology seems to lack the very useful, species-level, global catalogues available in paleobotany (U.S. Geol. Surv., 1955, no date a, b). This situation is worsened by the frequent lack of clear indications of a geologic time frame, the variable assignment of a locality's stratum to geologic periods (Jarzembowski, 1980), and/or the question of sample purity (Kühne \& Schlüter, 1985). The general topic of dating techniques has been thoroughly addressed by Dalrymple (1991). Occasionally, species are described but a family placement is not given (Zeuner, 1962). Furthermore, the depository and/or accession number of the specimens is often not stated, especially, but not exclusively, in the older papers. Knowing the collector is not necessarily a good indicator of the depository. For example, Scudder's collections of Florissant (Colorado, USA) fossils are deposited at the Museum of Comparative Zoology of Harvard University in Cambridge, MA, USA (Anonymous, 1988) but there are specimens also at the University of Colorado (Boulder, CO, USA), Princeton University (New Jersey, USA; Wickham, 1913a), the National Museum of Natural History (Washington, D.C., USA) (Grande, 1980), the American Museum of Natural History (New York, USA) and, apparently, at the Yale Peabody Museum (New Haven, CT, USA) (McLeod, pers. comm. 16 Dec. 1991). During the second half of the 19th century, bibliographic recycling (that is, the practice of having a paper published, almost unchanged, in several journals) was not uncommon, greatly complicating literature retrieval. Also, different authors entered journal citations in different, highly abbreviated, fashion. Finally, at times, the family name of an author is spelled in different ways (e.g. Rodendorf vs. Rohdendorf or Wejenbergh vs. Weyenberg or Medvediev vs. Medvedev or Martinov vs. Martynov); I choose to spell the name as it appears in the corresponding journal.)

To overcome some of these problems, several sources, such as, Commission de Stratigraphie (1956), Fairbridge and Jablonski (1979), Luttrell et al., 1986, Mulvihill (1982), Pearl (1951), Porter (1983), Sustrac (1984), Ward et al. (1981), and Wood et al. (1989) were recommended to me. To obtain the full citation of many 19th century papers, I relied on, amongst others, the British Museum, Natural History (1903), Royal Society of London (1867-1921), Scudder (1882, 1890c, 1891), and Spahr (1981a). Furthermore, I conducted several computer-assisted literature searches with combinations of the key words 'Chrysomelidae',
'Coleoptera', and 'fossil', using Agricola (1970-September 1992), BIOSIS (1989-July 1993), CAB (1984September 1992), GEOREF (1785-1992), and Life Sciences Collection (1989-June 1992). However, many papers and some taxa, hidden under articles whose main subject is fossil Coleoptera or fossil insects, may have escaped my detection. Although an invaluable and comprehensive source, the Zoological Record was only searched in part because of the projected, additional time investment required.

The taxonomy of fossil chrysomelids has several serious problems. Most of the records are very old and reflect the then available classifications or popular identification guides, such as: Fabricius $(1775,1792)$, Gyllenhall (1808-1827), Latreille (1819), Olivier (1791), Panzer (1795), Paykull (1800), Richardson (1837), and Ward (1776), among others. Some descriptions are very brief, for example, 'the species is very small' or 'one deeply punctured' (Hope, 1847). The quality of many descriptions and illustrations is inadequate, according to typical modern standards. However, there are some notable exceptions such as Förster (1891). Some identifications are, or were, in error (Birket-Smith, 1977; Ermisch, 1942; Oppenheim, 1887-1888), and some other classifications, such as the placement of Phalacrus in the Chrysomelidae (Berendt, 1845), have been abandoned by modern systems. Usually, owing to mediocre preservation, some fossils are identified, tentatively to genus, to family (Brodie, 1845; Göppert, 1855; Grande, 1980; Grimaldi \& Maisey, 1990; Meunier, 1898c; Phillips, 1871; Ponomarenko \& Schultz, 1988; Robert, 1838; Schawaller, 1986; Westwood, 1854) or in very general terms (Brongniart, 1827). Many others apparently have not been examined by specialists (Hieke \& Pietrzeniuk, 1984). Recently, I had the opportunity to examine Scudders's collection of fossil chrysomelids deposited at Harvard University and felt that, often, it is difficult to be certain whether a piece contains a chrysomelid at all. For example, a species of Trirhabda has been described based on antennal segments that can barely be seen. Currently, North American members of this genus can only be distinguished from several closely related genera by the relative length of several antennal segments (Wilcox, 1965; Hogue, 1970). Whalley (1985) said about the beetles found in the Lias (= European Jurassic) of Dorset, England, it is difficult, in many cases impossible, to place a single elytron (or even a pair of elytra) into a modern family reliably'. For example, many of the so-called Mesozoic chrysomelids may not be chrysomelids at all. (See Illustrations.) The most dramatic known case of misidentification are the plant remains considered by Heer as insects, including chrysomelids (Birket-Smith, 1977). Birket-Smith (1977) used the Latinized phrases nomen relictum, or nomina relictum, to indicate old names that now are regarded 
as misidentifications. These terms do not appear in the codes of Botanical or Zoological Nomenclature. Since long ago, it has been noted that, for the most part, amber-preserved insects can be identified to extant genera (Burmeister, 1836). However, unfortunately, amber deposits tend to be biased against larger organisms. On the other hand, the preservation of insect fossils deposited in the Oligocene-Eocene (2350 mya) rocks of Florissant is less complete.

\section{The fossil record}

\subsection{Paleozoic (570 to 245 mya)}

There seems to be no unequivocal fossil chrysomelid from the Paleozoic. Chrysomelids can be traced back only tentatively to the late Paleozoic to early Mesozoic (Triassic). This is in agreement with Rohdendorf (1956), Scudder (1887), Wootton (1981), and others, who placed the origin of the Phytophaga at the 'Permian-Triassic line'. Owing to the apparent absence of described Paleozoic fossil chrysomelids, it is very difficult to infer how common and speciose the family was in the late Paleozoic, as well as its affinities with closely-related beetles.

\subsection{Mesozoic (245 to 66.4 mya)}

There are, at least, 9 subfamilies, 19 genera, and 35 species of fossil chrysomelids reported from the Mesozoic.

Handlirsch recorded possibly the oldest known chrysomelid: Pseudochrysomelites rothenbachi Heer from a Trias. (=Triassic) Formation in Switzerland (Handlirsch, 1906-1908 a,b). Whether this taxon is conspecific with Chrysomelites rothenbachi Heer (1877), from the same formation, remains to be seen. This specimen, a right elytron (Fig. 29), is among the few Chrysomelids (or their parts) known from the Triassic. I consider this report questionable based on the lack of more conspecific material and of further study of the specimen.

Medvedev (in Rohdendorf, 1968) described several chrysomelid-like beetles that he assigned to the Protoscelinae. Protoscelines appeared in the late Jurassic and constitute the first good evidence of leaf beetles. However, 'some of Medvedev's Protoscelinae also show the long antennae and other cerambycid-like features' (Figs. 2-19; Crowson, 1975, 1981; Medvedev in Rohdendorf, 1968). The separation of cerambycid and chrysomelid lineages may date from the late Jurassic and may have been related to a division between Coniferae and Cycadeoids (cycad-like plants presumed to be ancestors of angiosperms) as basic food plants. Perhaps, protoscelines were visitors of cycads before the onset of angiosperms as has been suggested for Trigona bees (Ornduff, 1991). Medvedev (in Rohdendorf, 1968) believed that protoscelines are closely related to the Aulacoscelinae. Study of this material, in connection with the revision of the Aulacoscelinae, may be illuminating. Aulacoscelines, which have not yet been reported in the fossil record, are particularly interesting because they have been regarded as primarily cycad feeders (Monrós, 1954). Cycads (Stevenson, 1992) or cycadeoids were abundant in the Mesozoic, particularly in the Jurassic (Pant, 1973, 1988; Crowson, 1975, 1991), a fact that agrees with the inferred origin of the Aulacoscelinae. However, aulacoscelines not only visit cycads, but have been noted visiting, perhaps accidentally, plants in several families of angiosperms (Santiago-Blay, unpl. data). The Protoscelinae are also the first major evidence of the extinction, during the Cretaceous, of a Chrysomelidae lineage.

Eight extant subfamilies of chrysomelids are also represented in the Mesozoic fossil record: the Sagrinae, Clytrinae, Cryptocephalinae, Chrysomelinae, Eumolpinae, Galerucinae, Alticinae, and Cassidinae, most of which first appeared during the Jurassic.

The following 19 chrysomelid genera have been reported for the Mesozoic (subfamily parenthesized after a series of consubfamilials): Mesosagrites (Sagrinae), Cerambyomima, Protoscelis, Protosceloides, Pseudomegamerus (Protoscelinae), Clytra (as Melolontha?, Clytrinae), Cryptocephalus (Cryptocephalinae), Eumolpites (Eumolpinae), Chrysomela (most of them probably Chrysolina or Chrysomela sensu lato), Chrysomelites, Gonioctena, Oreina, Plagiodera, Timarchopsis (considered Coleoptera incertae sedis, Rohdendorf, 1957) (Chrysomelinae), Galerucites (Galerucinae), Altica (Alticinae), Cassida, Ditomoptera (Cassidinae), and Mesopleurites (uncertain subfamily placement).

Chrysomelids have been described for the Jurassic of the former Soviet Union and from western Mongolia. They are putatively similar to those present in the lower Cretaceous of those areas (Nikritin \& Ponomarenko, 1991; Kukalová-Peck, 1991). Curiously, no chrysomelids were reported for the former Soviet Union in the major work by Arnoldi et al., 1977.

Table 1 details the Mesozoic records and illustrations of fossil chrysomelids. Arrangement of subfamilies follows Seeno and Wilcox (1982).

\subsection{Cenozoic (66.4 mya to the present)}

There are, at least, 12 subfamilies, 121 genera, and 325 species of fossil chrysomelids reported for the Cenozoic. The newly represented subfamilies are the Donaciinae, Criocerinae, and the Hispinae. The increased number of taxa as geologic time approaches the present parallels that of many insect groups and 
comes as no surprise (Cockerell, 1925; Scudder, 1887). Most of the extant subfamilies of chrysomelids were represented in the Eocene (58-37 mya). Furthermore, according to Larsson (1978), many genera are represented in Cenozoic amber although relatively few have been described. Donaciines, a holartic, aquatic group (Borowiec, 1984), are the most common fossil chrysomelids (Debray, 1874). Larvae usually pupate in brown cocoons attached to the roots of their host plants, typically aquatic plants (particularly monocotyledoneous and presumed related dicots, such as the Nymphaeaceae) and not in a gas-filled chambers (Merritt \& Cummins, 1984).

Interestingly, some chrysomelid subfamilies have not yet been noted for the fossil record. These are the: Orsodacninae, Megascelinae, Synetinae, Megalopodinae, Chlamisinae, and Aulacoscelinae. All but the Chlamisinae are uncommon groups and their fossils are less likely to be collected, other things being equal. The Chlamisinae are more speciose, especially in the tropics. Additional sampling effort will probably disclose exemplars of these subfamilies.

The following 121 chrysomelid genera have been reported for the Cenozoic (subfamily parenthesized after a series of consubfamilials): Eosagra (Sagrinae), Donacia, Donaciella, Eodonacia, Haemonia, Hemidonacia, Macroplea, Neohaemonia, Plateumaris, Sominella (Donaciinae), Zeugophora (Zeugophorinae), Crioceridea, Criocerina, Crioceris, Electrolema, Lema (many now placed in Lema sensu lato, Criocerinae), Clytra, Clytrina, Labidostomis, Saxinis, Smaragdina? (Clytrinae), Cryptocephalites, Cryptocephalus, Pachybrachis (Cryptocephalinae), Adoxus, Bromius, Chalcosycia, Colaspis, Colasposoma, Eoeumolpinus, Eumolpites, Eumolpus, Graphops, Metachroma, Nodostoma, Pachnephorus, Profidia, Pseudocolaspis (Eumolpinae), Calligrapha, Chrysochloa (valid name is Oreina), Chrysolina, Chrysomela (many of the species now placed in Chrysolina or Chrysomela sensu lato), Chrysomelites, Chrysothoracus, Colaphellus, Crosita?, Eochrysomela, Eomelasoma, Gastroidea, Gonioctena, Gonocelis, Halocoleus, Hemisphaerocosites, Hydrothassa, Lina, Melasoma (valid name is Chrysomela), Oreina, Phaedon, Phratora, Phyllodecta (valid name is Phratora), Plagiodera, Prasocuris (=Hydrothassa?), Stenoplatys?, Strichosa, Zygogramma, Timarcha, cf. Zygospila (Chrysomelinae), Adimonia?, Agelasa, Agelastica, Diabrotica, Eogaleruca, Galeruca, Galerucella, Hadroscelus, Leptonesiotes, Lochmaea, Luperodes, Luperus, Monolepta, Ophraella, Pyrrhalta, Scelolyperus, Trirhabda (Galerucinae), Altica, Aphthona, Apteropeda, Asiorestia (as Crepidodera), Chaetocnema, Docemina (as Docemines), Hippuriphila, Longitarsus, Mantura, Ochrosis, Oedionychus, Oryctocirtites, Plectrotetrophanes, Prochaetocnema, Psylliodes, Systena, Walterianella (Alticinae), Anisodera, Anoplitis (a synonym of
Chalepus, many species listed in Anoplitis belong in Sumitrosis, both are New Word genera), Chalepus, Dicladispa, Hispa, ca. Microrhopala, Odontota, Oposispa, Protanisodera, Sucinagonia (Hispinae), Acassidites, Callistaspis, Cassida, Coelocassida, Delocrania, Eocassida, Inclusus, Mesomphalia, Oligocassida, and Paracassida (Cassidinae).

Table 2 details the Cenozoic fossil chrysomelids find and illustrations. The arrangement of subfamilies follows Seeno and Wilcox (1982). Airaphilus Redtenbacher, 1858 (see Ermisch, 1942) and Phloeonemites Wickham, 1912, both mentioned by Carpenter (1992), are reported to belong in the Cucujidae and Colydiidae, respectively.

\section{Extinctions and changes in geographical distribution}

The following 24 genera $(<1 \%$ of the extant) are possibly extinct: Eosagra Sagrinae), Criocerina, Electrolema (Criocerinae), Clytrina (Clytrinae), Cryptocephalites (Cryptocephalinae), Eoeumolpinus, Eumolpites (Eumolpinae), Chrysomelites, Eochrysomela, Gonocelis, Halocoleus, Hemisphaerocosites (Chrysomelinae), Galerucites, Hadroscelus (Galerucinae), Oryctoscyrtetes Plectrotetrophanes (Alticinae), Sucinagonia, Oposispa, Protanisodera (Hispinae), Acassidites, Callistaspis, Coelocassida, Eocassida, and Inclusus (Cassidinae) (cf. Seeno \& Wilcox, 1982). However, further studies may disclose members of some of these genera still living today, particularly in the tropics (Gressitt, 1963). Also, after revisionary works, many of these 'extinct' genera may actually be classified as members of extant genera.

Other genera seem to have become rare or extinct in areas that they previously occupied. For example, Delocrania (Cassidinae) (Farell et al., 1992; Morell, 1992) Leptonesiotes Galerucinae), and Walterianella (Alticinae) have been found in Dominican amber but they have not been reported for the extant Hispaniolan chrysomelid fauna.

Insect low extinction ratio over evolutionary time (Labandeira and Sepkoski, 1993) may serve as a baseline of comparison with modern, mostly anthropically-caused, extinction rates.

\section{Biogeography and paleoclimatology}

Fossils provide factual data on where taxa lived in the past (Schwert \& Ashworth, 1988). Also, they document places where large faunas that once existed are now absent (Heer, 1876).

One of the best examples is the entomofauna of the Shanwang fossil beds (mid Miocene), located in the 
People's Republic of China. This deposit shares less taxa with its extant local counterparts. However, when compared with younger fossil beds, the Shanwang shows the strongest affinities with the Florissant (late Oligocene), and less similarities with the Baltic fauna (late Eocene to early Oligocene) (Zhang, 1989). This broad Holarctic biotal similarity has been noted repeatedly for many other groups (e.g. Ornduff, 1974).

In general, the fossil record of chrysomelids appears to be dominated by relative stasis, with occasional spurts of extinctions in some groups and speciation in others. For instance, the donaciine fossil species, Plateumaris primaeva (Wickham, 1912) and Donacia wightoni (Askevold, 1990a), members of inferred relatively derived groups, have not changed much morphologically, suggesting relative stasis (Askevold, 1990a, 1991). The origin of the lineage comprising these species groups has been dated at, approximately, 40-60 mya (Askevold, 1991).

Owing to the lack of geologic series of any chrysomelid taxon, the stratophenetic method of circumscribing taxa and suggesting classifications (Gingerich, 1979) is of little use for the family. Others, such as Stevens (1980) believed that the fossil record is almost useless in cladistic analyses due to its incompleteness. In addition, some have said that, even assuming a perfectly complete fossil record, it would be impossible to infer a logically necessary ancestor-descendant relationship among three or more taxa because the spatio-temporal connection between fossils is a construct that investigators overlay to the specimens. The consequences of this kind of epistemology are fatal to anyone trying to do historical reconstructions.

Insect assemblages, including chrysomelids, have been used to assess paleoenvironmental conditions and community structure (Ashworth et al,, 1981; Böcher, 1989; Elias, 1982, 1985, 1991, 1992; Elias \& van Devender, 1990; Elias et al., 1992; Fujiyama, 1980; Kimoto, 1981; Nasarow, 1984; Nel, 1988; Schwert \& Ashworth, 1988, 1990; Schwert \& Morgan, 1980; Whitehead, 1989; and many others). Dispersal allows insects to satisfy their ecological and physiological requirements (Coope, 1970; Elias, 1991; Schwert \& Ashworth, 1988, 1990). Apparently, judging from the places chrysomelids have lived, ecological requirements have remained similar to those of modern faunas (Ashworth \& Hoganson, 1983; Coope, 1970, 1978, 1979a, 1979b; Zhang, 1989). Evidently, chrysomelids and many other insects, have adapted to this by shifting their geographical ranges. Kimoto (1981) used donaciines and $\mathrm{Nel}$ (1988) hispines to assist determining paleoenvironmental conditions. However, similarity of faunas is not necessarily an indication of synchronous climate resemblance (Wickham, 1917). Faunal data, together with pollen analyses, are more reliable for inferring paleoclimatological variations, such as in
North America (Baker et al., 1991; Elias \& Short, 1992; Elias \& Van Devender, 1992; Elias et al., 1986; Elias \& Wilkinson, 1983; Morgan et al., 1986; Pearson, 1962; Pilny \& Morgan, 1987; Schwert, 1992; Short \& Elias, 1987; Short et al., 1992), Chile (Hoganson \& Ashworth, 1992), Europe (Pearson, 1962; Ponel \& Coope, 1990); and in the former Soviet Union (Rohdendorf et al., 1980). Some recent distribution patterns of beetles, including chrysomelids, have apparently developed only since the Quaternary (Ashworth \& Hoganson, 1987; Girling, 1976, 1977, 1978; Kenward, 1978).

In spite of large climatic oscillations, there seems to have been little modification of the Chrysomelidae during the Cenozoic; the most dramatic change is the apparent extinction of several genera, including some known only from the Cenozoic (Coope, 1978, 1979a, 1979b). However, due to dispersal, extinction is not a necessary an indication that the extant fauna is only a remnant of a putatively much larger fauna (Askevold, 1991). For example, Hemidonacia insolita, does not occur today in the western Paleartic region where the fossils were found (Askevold, 1991).

\section{Feeding preferences and other biological features}

The biological information inferred from the available data suggests that the chrysomelid-plant associations were established, at least, in the late Mesozoic to early Cenozoic. However, it is 'difficult to say what leaves they [phytophagous insects, including chrysomelids] had fed upon' (Heer, 1876). Some donaciines from the Paleocene were found associated with water-lily fossils (Nymphaeaceae) (Uhmann, 1939). Recent donaciines are common inhabitants of water lilies and other aquatic plants (Schneider \& Moore, 1977). Probably due to the co-occurrence of fossil plants and fossil chrysomelids, Heer (1876) speculated that species of Donacia would have been found 'sitting on flowers of rushes' and feeding on $J$ uncus, while $D$. palaemonis Heer apparently fed on reeds and rushes. Plateumaris braccata (Scopoli, 1772) lived on Iris pseudoacorus (Pawłowski et al., 1987) and D. tomentosa possibly lived on Butomus umbellatus. Lema vetusta Heer (Criocerinae) probably lived on liliaceous plants (Heer, 1876). Gonioctena curtisi Oustalet, 1874 (Chrysomelinae) fed on Populus spp. like the extant $G$. decemnotata Marsham (=G. rufipes DeGeer). Heer $(1872,1876)$ suggested that C. calami Heer fed on rosales and spent time 'sunning themselves on reeds' and that Chrysomela populeti (Heer) (as Lina) most probably ate poplar and willow leaves (Heer, 1876). Phyllobrotica (Galerucinae) beetles and the Labiaceae host plants are believed to have been associated, at least, since the mid-Tertiary (Farrell \& Mitter, 1990). Eggs of alticines, perhaps Altica, have 
been found on fossilized, middle Eocene Alnus (Betulaceae) leaves, a host-plant relationship that still persists (Lewis \& Carroll, 1991). 'Anoplitis' bremii Heer (Hispinae) is presumed to feed on pomaceans (Rosaceae), such as Pyrus and Amelanchier, in the Tertiary of Switzerland and Cassida hermione Heer, 1847 and C. blancheti Heer, 1856a possibly fed on thistles (Asteraceae) (Heer, 1876).

Some host plant information seems to lack credibility. For example, according to (Bachofen-Echt, 1949), Electrolema baltica Schaufuss, 1892 'apparently lived in the needles of the amber tree'. This species possibly did not live in association with the amber tree because many Criocerinae are associated with monocots and, occasionally, with dicots (Larsson, 1978; Schmitt, 1988). The same probably holds true for the elytra of Plateumaris discolor (Panzer, 1795) that have been associated with glacial deposits containing remains of Pinus (Pinaceae), Populus (Salicaceae), and Alnus (Lortet \& Chantre, 1872). Many chrysomelids that have been found in amber more likely got stuck in the resin (Poinar, 1992) and were not associates of the resin-producing plant.

Very little information is known or has been inferred about other aspects of the biology of fossil chrysomelids (Scudder, 1887; Heer, 1872, 1876). Donaciines are the most common identifiable chrysomelids in the Cenozoic, particularly the Quaternary deposits and, as their extant counterparts, they have been assumed to be aquatic (Blair, 1923-1924). Heer (1876) noted that, surprisingly, donaciines were rare in the chrysomelidrich deposits of Lake Oeningen, in Switzerland. Amber preservation is so good that identified larvae have occasionally been mentioned, such as three Chrysomela (sensu lato, Menge, 1856) and cocoons of Donacia (Henriksen, 1914). A larval case assigned to Clytra carbonaria Heyden is illustrated showing that this feature of its life history was fully developed millions of years ago (Heyden \& Heyden, 1865). I have seen larval cases that probably belong in one of the subfamilies in the old Camptosomes or Camptosomata.

There is almost no data available on the terrestrial biomes that fossil chrysomelids inhabited. Pachybrachis sp. (Cryptocephalinae), Strichosa eburata Blanch. (Chrysomelinae), several species of Altica and other alticines, as well as a species in a genus close to Asiorestia (Alticinae) have been associated with Quaternary forest vegetation; other genera have been connected with aquatic/shoreline vegetation (Hoganson \& Ashworth, 1992).

\section{Acknowledgments}

I wish to express my deepest gratitude to I. Radkey and $\mathrm{N}$. Axelrod, as well as to the kind staff of the
Earth Sciences, Biosciences, Main Libraries, and the Interlibrary Borrowing Service (University California, Berkeley, CA, USA) for their invaluable assistance in finding, or requesting for me, many of the rather obscure works herein referenced. F. M. Carpenter (Harvard University, Cambridge, MA, USA), from whom I have learnt a lot about fossil insects, put Scudder's collection at my disposition, made available a draft of the Chrysomelidae section from his work on fossil insects, and assisted me in obtaining some references. His recently published tomes (1992) arrived in my mail a day before I finished editing the first version of this chapter and it proved useful. A. C. Ashworth (North Dakota State Univ., Fargo, USA), S. A. Elias (Institute of Arctic and Alpine Research, Boulder, $\mathrm{CO}$ ) and S. J. Seybold (Univ. California, Berkeley) made me aware of many papers, especially those from the huge Quaternary insect literature. J. Baxter (Univ. California, Berkeley) translated several papers written in the old German calligraphy and some papers in Cyrilic. J. Foss and A. Urbanic (Univ. California, Berkeley) also translated some papers in Cyrilic. C. C. Resting-Jeppesen (Charlottenlund, Denmark) current Editor of Acta Arctica, allowed me to use the photographs from Birket-Smith's (1977) paper. K. Cirina, from University of California Press granted permission to use the line drawings from Gressitt's (1971) paper. To save time, most editors or authors of some papers published within the last 25 years were contacted to request permission for the use of illustrations from their publications. The sources of illustrations have been acknowledged. Dr. D. Wood (Univ. California, Berkeley) very kindly covered part of the photographic costs of this chapter. Drs. N. Berti (Museum d'Histoire Naturelle, Paris), M. Biondi (Instituto di Zoologia, Roma), L. Borowiec (Zoological Institute, Univ. Wroclaw, Poland), M. Cox (Natural History Museum, London), R. A. Crowson (Univ. Glasgow, Scotland), M. Daccordi (Museo Civico di Storia Naturale, Verona, Italy), D. G. Furth (Museum of Comparative Zoology, Harvard University, Cambridge, MA), B. Gruev (Plovdiv, Bulgaria), P. H. A. Jolivet (Paris), L. LeSage (Biosystematic Research Institute, Ottawa), I. Lopatin (Belorussia State Univ., Minsk), G. O. Poinar, Jr. (Univ. California, Berkeley), E. G. Riley (Texas A \& M Univ., College Station, TX), G. A. Samuelson (B. P. Bishop Museum, Honolulu, HI), M. Schmitt (Museum Koenig, Bonn), and C. L. Staines (Edgewater, MD, USA), read a copy of the entire typescript, suggested numerous modifications, and made constructive criticisms. Although I am indebted with them all, the involuntary errors and inevitable omissions that this contribution still contains, which I will appreciate being notified of, are my responsibility. 
Table 1. Mesozoic records of fossil Chrysomelidae

\begin{tabular}{cccc}
\hline Taxon, author, year & Period & Location & Depositorics, references, other \\
\hline
\end{tabular}

\section{Sagrinae}

Mesosagrites multipunctatus Martynov, 1935 (Fig. 1)

\section{Protoscelinae}

Cerambyomima longicornis Medvedev (in Rohdendorf, 1968) (Fig. 2)

Protoscelis jurassica Medvedev (in Rohdendorf, 1968) (Figs. 3-10)

Protosceloides nitidicornis Medvedev (in Rohdendorf, 1968) (Fig. 12-14)

P. parrula Medvedev (in Rohdendorf, 1968) (Fig. 11)

Pseudomegamerus grandis Medvedev (in Rohdendorf, 1968) (Figs. 15-17)

\section{Clytrinae}

Clytra sp. (as Melolontha?)

\section{Cryptocephalinae}

Cryptocephalus antiquus Weyenbergh, 1869a (Fig. 18)

C. mesozoicus Weyenbergh, $1869 \mathrm{a}$ (also as Cryptocephalus mesozoïcus) (Fig. 19)

$$
\text { Cryptocephalus sp. }
$$

\section{Eumolpinae}

Eumolpites jurassicus Martynov, 1926 (Fig. 20)

Eumolpites liberatus Heer, 1865 (Fig. 21)

\section{Eumolpites sp.}

possible Eumolpinae, resembling Pilacolaspis (Figs. 240, 241, the later a SEM from a silicon rubber cast)

\section{Chrysomelinae}

Chrysomela andraei Brodic, 1845 (valid name for many species on this genus is Chrysolina)
C. dubia Westwood, 1854
C. dunkeri Westwood, 1854

Lower Jurassic

Jurassic

Jurassic

Jurassic

Jurassic

Jurassic

Jurassic

Jurassic horizon

not found

Jurassic

Jurassic to Tertiary

Lias (Jurassic)

Upper Cretaceous

West Cracombc, Worcestershire, England

\section{Solnhofen, Bavaria}

Solnhofen, Bavaria

Oölite of Bavaria; England

slates of east Kara Tau Kazaktstan, Central Asia

Switzerland

Schabelen, England

Hawkes Bay, New Zealand

Lower Lias (= European Jurassic) horizon

lower Purbecks horizon, Durdlestone Bay, England Jurassic?

lower Purbecks horizon, Jurassic?

Forthampton, England, type: no 33 , only known from a single damaged elytron, doubtful placement; Hennig, 1981

speciments 2066/3241 at Palcontology Institute of the Academy of Sciences, Moscow

specimens 2066/3311, 2066/3000, $2239 / 923,2239 / 1342$, and $2384 / 770$ at Paleontology Institute of the Academy of Sciences, Moscow; Crowson, 1975, 1981

specimen 2066/3311 at Paleontology Institute of the Academy of Sciences. Moscow

specimen 2066/2593 at Paleontology Institute of the Academy of Sciences, Moscow

specimens 2066/2593 at Paleontology Institute of the Academy of Sciences, Moscow

Phillips, 1871

Weyenbergh, 1869a, 1869b, 1874

some specimens at Paleontology Museum, Munich; Meunier, 1898c; Oppenheim, 1887-1888; Weyenbergh, $1869 \mathrm{a}, 1869 \mathrm{~b}$

Scudder, 1887

collections of the Geological Committee former Soviet Union; possibly not a chrysomelid (Crowson, pers. comm. Jan. 1993)

Hecr, 1872, 1876

Heer, several papers; Scudder, 1887

New Zealand Geological Survey Coll. (AR 1421); Craw and Watt. 1987

Giebel, 1856; Scudder, 1887

Giebel, 1856

Gicbel, 1856 
Table 1. (Continued)

\begin{tabular}{|c|c|c|c|}
\hline Taxon, author, year & Period & Location & Depositories, references, other \\
\hline C. ignota Westwood, 1854 & $\begin{array}{l}\text { lower Purbecks horizon, } \\
\text { Jurassic? }\end{array}$ & Durdlestone Bay, England & Giebel, 1856 \\
\hline Chrysomela spp. & Lias horizon (Jurassic) & Oölite, Bavaria; and England & $\begin{array}{l}\text { Heer, 1876, 1879, 1883b; Scudder, } \\
1887\end{array}$ \\
\hline $\begin{array}{l}\text { Chrysomelites jurassicus Oppenheim, } \\
1887-1888 \text { (Figs. 22-23) (Fig. } 22 \\
\text { from Meunier, 1898c). }\end{array}$ & Jurassic & Eichstätt, Bavaria & $\begin{array}{l}\text { some specimens at Paleontology } \\
\text { Museum, Munich; Meunier, } 1898 \mathrm{c}\end{array}$ \\
\hline C. liasina Brodie, 1845 & $\begin{array}{l}\text { Lower Lias horizon (Juras- } \\
\text { sic) }\end{array}$ & Hasfield, England & Giebel, 1856 \\
\hline $\begin{array}{l}\text { C. lithographica Weyenbergh, } 1869 \mathrm{a} \\
\text { (Figs. } 24,25 \text { ) }\end{array}$ & Jurassic horizon & Solnhofen, Bavaria & Weyenbergh, 1869b, 1874a, 1874b \\
\hline C. macrothoracicus Oppenheim & Mesozoic & $\begin{array}{l}\text { possibly Eichstätt, Bavaria, } \\
\text { Germany }\end{array}$ & $\begin{array}{l}\text { some specimens at Paleontology } \\
\text { Museum, Munich; Meunier, } 1898 \mathrm{c}\end{array}$ \\
\hline $\begin{array}{l}\text { C. minima Oppenheim, } 1887-1888 \\
\text { (also as Chrysomelites minimus) (Fig. } \\
\text { 26) }\end{array}$ & Jurassic horizon & Eichstätt, Bavaria & $\begin{array}{l}\text { some specimens at Paleontology } \\
\text { Museum, Munich; Scudder, 1891; } \\
\text { Meunier, 1898c }\end{array}$ \\
\hline C. prodromus Heer, 1865 (Fig. 27) & Lias horizon (Jurassic) & Schambelen, Switzerland & $\begin{array}{l}\text { Heer, 1872, } 1876, \quad 1879, \quad 1883 b ; \\
\text { Scudder, } 1885,1886\end{array}$ \\
\hline $\begin{array}{l}\text { C. rura Weyenbergh, } 1869 \text { a (also as } \\
\text { Chrysomela rara) (Fig. 28) }\end{array}$ & Jurassic horizon & Solnhofen, Bavaria & Weyenbergh, $1869 b, 1874$ \\
\hline $\begin{array}{l}\text { C. rothenbachi Heer, } 1877 \text { ( =? Pseu- } \\
\text { dochrysomelites rothenbachi) (Fig. 29) }\end{array}$ & Trias. (= Triassic) horizon & Rütihard, Basel, Switzerland, & Handlirsh $1906-1908 \mathrm{a}, \mathrm{b}$ \\
\hline Chrysomelites sp. & Triassic; Lias (Jurassic) & $\begin{array}{l}\text { Lettenkohle of Rütihard, } \\
\text { Basel; England, Schambelen }\end{array}$ & $\begin{array}{l}\text { Heer, 1876, 1879, 1883b; Scudder, } \\
1887\end{array}$ \\
\hline $\begin{array}{l}\text { Gonioctena curtisii Oustalet, } 1874 \\
\text { (Gonictena and Gonictema are mispel- } \\
\text { lings) }\end{array}$ & not found & not found & Flach, 1884 \\
\hline Oreina sp. & Oligocene to Miocene & Germany & Förster, 1891; Hcer, 1847 \\
\hline Plagiodera sp. & Oligocene & France; Germany & $\begin{array}{l}\text { Heyden and Heyden, 1866; Théobald, } \\
1937\end{array}$ \\
\hline $\begin{array}{l}\text { Timarchopsis czckanowskii Brauer, } \\
\text { Redtenbacker, and Ganglbauer, } 1859\end{array}$ & Lias (Jurassic) horizon & Ust Balci. Siberia & $\begin{array}{l}\text { considered Coleoptera incertae sedis, } \\
\text { Rohdendorf, 1957: according to } \\
\text { Jolivet, pers. comm. it resembles } \\
\text { Timarcha. }\end{array}$ \\
\hline $\begin{array}{l}\text { Galerucinae } \\
\text { Galerucites carinata Oppenheim. } \\
1887-1888 \text { (also as G. carinatus) (Fig. } \\
30 \text { ) }\end{array}$ & Jurassic horizon & Kelheim, Bavaria & $\begin{array}{l}\text { some specimens at Paleontology } \\
\text { Museum, Munich; Meunier, 1898c; } \\
\text { Oppenheim, 1887-1888; Scudder, } \\
\text { 1891; possibiy a heteropteran }\end{array}$ \\
\hline $\begin{array}{l}\text { Alticinae } \\
\text { Alicica sp. }\end{array}$ & $\begin{array}{l}\text { Lower Purbecks horizon, } \\
\text { Jurassic? }\end{array}$ & Durlestone Bay, England & Westwood, 1854 \\
\hline \begin{tabular}{l}
\multicolumn{3}{c}{ Cassidinae } \\
Cassida aequinoca Weyenbergh, \\
1869a (Fig. 31)
\end{tabular} & Jurassic horizon & Solenhofen, Bavaria & $\begin{array}{l}\text { Giebel, 1856; Handlirsch, 1906-1908a, } \\
\text { 1906-1908b; Scudder, 1891; Weyen- } \\
\text { bergh, } 1869 \mathrm{~b}, 1874\end{array}$ \\
\hline Cassida nr. meridionalis & not found & not found & Serres, 1829 \\
\hline C. nr. viridis (Dejean) & not found & not found & Serres, 1829 \\
\hline Cassida sp. & not found & Oölite of Bavaria & Scudder, 1887 \\
\hline Ditomoptera dubia Germar, 1839 & Jurassic horizon & $\begin{array}{l}\text { Solenhofen, Bavaria, Ger- } \\
\text { many }\end{array}$ & $\begin{array}{l}\text { a synonym of Ditomoptera dubia } \\
\text { Deichmüler 1896: Assmann, 1870; } \\
\text { Giebel, 1852; Oppenheim, 1887- } \\
\text { 1888; Weijenbergh, } 1869 \mathrm{~b}\end{array}$ \\
\hline
\end{tabular}


Table 1. (Continued)

\begin{tabular}{lcccc}
\hline \multicolumn{1}{c}{ Taxon, author, year } & Period & Location & Depositories, references, other \\
\hline $\begin{array}{l}\text { Uncertain subfamily placement } \\
\begin{array}{l}\text { Mesopleurites jurasicus Martynov, } \\
1926\end{array}\end{array}$ & Jurassic & $\begin{array}{l}\text { Malm of Karatau, former } \\
\text { Soviet Union }\end{array}$ & Rohdendorf, 1962 \\
$\begin{array}{l}\text { Unidentified "chrysomelids" (Fig. } \\
\text { 32) }\end{array}$ & Lias (Jurassic) horizon & $\begin{array}{l}\text { Apperley, } \\
\text { Forthampton, Hasfield, } \\
\text { Stonefield Slatc. England }\end{array}$ & Brodie, 1845; Phillips, 1871 \\
\hline
\end{tabular}

Table 2. Cenozoic records of fossil Chrysomelidae

\begin{tabular}{|c|c|c|c|}
\hline Taxon, author, year & Period & Location & Depositories, references, other \\
\hline \begin{tabular}{l}
\multicolumn{2}{c}{ Sagrinae } \\
Eosagra obliquata Haupt, 1950 (Fig. \\
33)
\end{tabular} & Tertiary to middle Eocene & Geiseltal, Germany & specimens 2610,5871 ; Crowson, 1975 \\
\hline $\begin{array}{l}\text { E. subparallela Haupt, } 1950 \text { (Figs. 34- } \\
\text { 36) }\end{array}$ & Tertiary to middle Eocene & Geiseltal, Germany & $\begin{array}{l}\text { specimens } 1249,1858,2205,2302 \\
2485,2654,2720,3303, \text { and } 3385 \\
\text { Crowson et al., } 1967\end{array}$ \\
\hline Eosagra sp. (Figs. 37-39) & Eocene & Geiseltal, Germany & $\begin{array}{l}\text { types, G } 55 / 85,86 a b, 87,88 \text {; others } \\
\text { specimens: G 55/89-55/94. Sammlung } \\
\text { des Geologisch-Paläontologischen } \\
\text { Instituts der Universität Halle, } \\
\text { Germany; Haupt, 1956; Zherikhin, } \\
1970\end{array}$ \\
\hline
\end{tabular}

\section{Donaciinae \\ Donacia aquatica Linné, 1758 (also} as $D$. acuatica)

$$
\text { D. aequalis Say }
$$

D. bidens Olivier

D. bicolor Zschach, 1788 (Fig. 41) (also as D. bicolora)

D. biimpressa Melsch.

D. brevicornis Ahrens, 1810

$D$. cincticornis Newman or D. proxima Kirby

D. conelli Cockerell, 1927

D. (Donacia) crassipes Fabricius, 1775 (possible mispelled as $D$. cryssipes in Nasarow, 1984)
Quaternary

Quaternary

Quaternary

Holocene

Quaternary

Quaternary

Holocene

Pleistocene

Quaternary
West Cumberland, England; widespread in Findland; Schonen, Sweden; Denmark and southern Sweden

Innerkip, Ontario, Canada

Schonen, Sweden

West Cumberland, England; Belorussia, former Soviet Union

Innerkip, Ontario, Canada

southeast Finland

Gervais Formation, Minnesota, USA

interglacial, Cordova Bay, Victoria, Vancouver Island, Canada,

Mundesley, England; Vanne, France; Denmark and southern Sweden; Nordost Seeland, Denmark; SumpflandFauna; mid-Finland
(=? D. dentipes Fabricius); Henriksen, 1933; Kurck, 1917; Pearson, 1962 Poppius, 1911

Quaternary Entomology Laboratory, University of Waterloo Canada; Pilny and Morgan, 1987

Kurck, 1917

some specimens probably at Paleontomological Institute of the Academy of S.iences, Moscow; Goecke, 1943; Nasarow, 1984; Pearson, 1962

Quaternary Entomology Laboratory, University of Waterloo Canada; Pilny and Morgan, 1987

Poppius, 1911

Ashworth, 1980

Goecke, 1960a; Wickham, 1933

some specimens probably at the Paleontomological Institute of the Academy of Sciences, Moscow (9/274); Bell, 1888; Fliche, 1876; Henriksen, 1933; Jessen, 1920; Meunier, 1901; Poppius, 1911; Nasarow, 1984; Schäff, 1892 
Table 2. (Continued)

\begin{tabular}{|c|c|c|}
\hline Taxon, author, year & Period & Location \\
\hline $\begin{array}{l}\text { D. curticollis Haupt, } 1956(=D . \\
\text { geiseltali Goecke, 1959?) }\end{array}$ & Eocene & Geiseltal, Germany \\
\hline D. dentata Hoppe, 1795 & Quaternary & $\begin{array}{l}\text { Denmark and southern } \\
\text { Sweden; Nordost Seeland, } \\
\text { Denmark }\end{array}$ \\
\hline D. disjecta Förster, 1891 (Fig. 42) & Oligocene & $\begin{array}{l}\text { Brunnstadt Alsace-Lorraine, } \\
\text { France }\end{array}$ \\
\hline D. distincta LeConte, 1850 (Fig. 43) & Quaternary & $\begin{array}{l}\text { Lake Agassiz, Minnesota, } \\
\text { USA; Umiakoviarusek, } \\
\text { Northeastern Labrador, } \\
\text { Canada; Innerkip, Ontario, } \\
\text { Canada }\end{array}$ \\
\hline
\end{tabular}

D. dubia Théobald, 1937 (Fig. 242) Oligocene

D. elongatula Scudder, 1900 (Fig. 44)

D. extincta Kolbe, 1933

D. fennica Paykull, 1800

D. cf. fuegens LeConte

D. genin Mortillet, 1850 (also as $D$. genini)

D. hirticollis Kirby

D. hydrocaridis? Fabricius

D. impressa Payskull, 1799

D. jaroslavii Lomnicki, 1894 (Fig. 45)

D. laevicollis? Thomson

D. letzneri Assmann, $1870(=$ ? D. limbata Panzer) (Fig. 46)

D. lignitum Sordelli, 1882 (Fig. 47)

D. limbata $(=$ ? D. lemnae Fabricius)

D. marginata Hoppe, 1795

D. menyanthidis Fabricius

D. minuta Haupt, 1956 (Fig. 48)

D. obtusa Haupt, 1956 (also as D. obstusa)
Kleinkembs, France

Pleistocene

Quaternary

Pleistocene

Quaternary

Quaternary

late Quaternary

Quaternary

Quaternary

Pleistocene

Quaternaıy

Oligocene

Pleistocene

not found

Holocene

Quaternary

Eocene

Eocene sachusetts, USA southern Sweden;

Hösbach in Bayern

Sonnaz, France Montana, USA and southern Sweden izien, Poland many

$$
\text { Leffe, Italy }
$$

Lombardia, Italy Union

\section{Greifswald, Germany}

Geiseltal, Germany

Geiseltal, Germany
Depositories, references, other

type, G 55/76, Sammlung des Geologisch-Paläontologischen Instituts der Universität Halle, Germany; Goecke, $1960 a$

Henriksen, 1914, 1933; Jcssen, 1920

Goeckc, 1960a; Théobald, 1937

Quaternary Entomology Laboratory, University of Waterloo Canada; Ashworth, et al., 1972; Elias, 1982; Pilny and Morgan, 1987

R 698, Coll. Mieg du Museum de Bâle; Théobald, 1937

Fort River, Hadley, Old Hampshire Co., Mas-

spätglacial, Toppeladugard,

Sweden; Denmark and Innerkip, Ontario, Canada

False Cougar Cave, Boreal

south Schonen, Sweden

Schonen, Sweden; Denmark

Ozokerritton, Baryslaw, Gal-

$$
\text { south Schonen, Sweden }
$$

Schossnitz, Schlesien, Ger-

West Cumberland, England; Belorussia, former Soviet
Goecke, 1943, 1960a; Scudder, 1898. 1900; Wickham, 1920

Goecke, 1960a; Holst, 1908; Henriksen, 1933; no. 11

Flach, 1884, Meunier, 1901

Quaternary Entomology Laboratory, University of Waterloo Canada; Pilny and Morgan, 1987

Goecke, 1960a; Meunier, 1884

Elias, 1991

not found

Andersson, 1889; Kurck, 1917; Henriksen, 1933

Goecke, 1943, 1960a

Andersson, 1889

Goecke, 1943, 1960a

Goecke, 1943, 1960a

Sordelli, 1882a

some specimens probably at the Paleontomological Institute of the Academy of Sciences, Moscow; Nasarow, 1984; Pearson, 1962

Chamisso, 1824; Schaff, 1892

type, G 55/78, Sammlung des Geologisch-Paläontologischen Instituts der Universität Halle, Germany; Goecke, 1960a

type, G 55/77, Sammlung des Geologisch-Paläontologischen Instituts der Universität Halle, Germany; Goecke, 1960a 
Table 2. (Continued)

$\frac{\text { Taxon, author, year }}{\text { D. obscura (also as D. oscura) Gyl. }}$
lenhal, 1813 (Fig. 49)

D. palaemonis Heer, 1847 (Fig. 50)

D. parvula Heer, 1870 ("nomina relictum", Birket-Smith, 1977) (Figs. 51, 88 A-D; Fig. 50 from Goecke, 1943; others from Birket-Smith, 1977)

$$
\text { D. piscatrix Lacordaire }
$$

D. pitoni Goecke, 1959? (= D. antiqua Piton (in Piton and Théobald, 1935) (Fig. 40)

$$
\text { D. polita Kunze, } 1818
$$

D. pompatica Scudder, 1890 b (Fig. 52) [(close to $D$. pubicollis (Suffrian, 1872)]

$$
\text { D. proxima Kirby }
$$

D. pterobrachys Haupt, 1956 (also as D. pterobrachis) (Fig. 53)

$$
\begin{aligned}
& \text { D. pubescens LeConte } \\
& \text { D. pusilla LeConte }
\end{aligned}
$$

D. rottensis Goecke, 1960b

D. sagittariae Fabricius, $1792[=$ ? Donacia atrea Hoppe, $=$ ? D. bicolora Zschach, 1788] (Fig. 54)

D. semicuprea Panzer, 1795 (Fig. 55)

D. simplex Fabricius, $1775 \quad\left[\begin{array}{lll}? & D . & \text { Pliocene - Quaternary }\end{array}\right.$ linearis Hoppe 1795]

D. simittiana Heer, 1870 ("nomina relictum", Birket-Smith, 1977) (Figs. 56 and 89$)$
Miocene

Eocene to Miocene

Quaternary

Miocene to Quaternary

Quaternary

Quaternary

Quaternary

Eocene

Holocene

Pleistocene

Quaternary

Eocene to Miocene
Holocene

Oligocene

\section{Location}

West Cumberland, England; Hösbach, Bayern, Germany; valley of the Seine, Paris; Denmark, Schonen and southern Sweden; widespread in Finland

Lake Oeningen, Baden,

Spitsbergen, Grönland

Innerkip, Ontario, Canada

Cinérites de Varennes, L'Auvergne, Masif Central, France

\section{Belle-Ile}

interglacial deposits: Scar.borough. Ontario; Cordova Bay, Victoria, Vancouver Island, Canada, Illinois, USA; Innerkip,
Ontario, Canada

Geiseltal, Germany

Lake Agassiz, Minnesota, USA

Umiakoviarusek, Northeastern Labrador, Canada

\section{Rott, Germany}

Pre- and Interglacial, Launberg, Elbe; Hösbach, Bayern, Germany

Denmark and southern Sweden; Belorussia, former Soviet Union

Wester Cumberland peat, Wolvercote, Oxfordshire, Norfolk forest bed (preglacial), and Mundesley, England; Dogger Bank, North Sea, long. $2-5 \mathrm{E}$, lat. $54-56^{\circ} \mathrm{N}$, ca. $200 \mathrm{~km} \mathrm{NNE}$ London; Denmark and southern Sweden; Poland?

Spitsbergen, Grönland
Depositories, references, other

Flach, 1884; Goecke, 1943; Henriksen, 1933; Holst, 1908; Jessen, 1932; Kurck, 1917; Lesne, 1925; Meunier, 1901; Pearson, 1962; Poppius, 1911; Westergard, 1912

Giebcl, 1852; Goecke, 1943, 1960a; Heer, 1847, 1876, 1883b

Goecke. 1943, 1960a

Quaternary Entomology Laboratory. University of Waterloo Canada; Pilny and Morgan, 1987

no. 6, Station Limnologique de Besse; a Donacia antiqua has already been named!; Kunze, 1818; Goecke, 1960a

\section{Lesne, 1918a, b}

variable in color, from purple to green, specimens 14566, 14577 , 14582, 14573, and 14581; Cockerell, 1927; Goccke, 1943, 1960a; Scudder, 1890b, 1895a 1895; Wickham, 1920

Quaternary Entomology Laboratory, University of Waterloo Canada: Pilny nad Morgan. 1987; Baker. 1920; Wickham, 1927

type, G 55/80, Sammlung des Geologisch-Paläontologischen Instituts der Universität Halle, Germany; Goecke, 1960a

$$
\text { Ashworth et al, } 1972
$$

Elias, 1982

not found

Flach, 1884; Meunier, 1900, 1901

some specimens probably at the Paleontomological Institute of the Academy of Sciences, Moscow; Goecke, 1943; Henriksen, 1933; Jessen, 1932; Nasarow, 1984

some specimens possibly at Institute of Systematic and Experimental Zoology, Polish Academy of Sciences, Kraków; Bell, 1888: Bell and Bell, 1873; Blair 1923-1924; Pawlowski et al., 1987; Pearson, 1962; Poulton, 1923-1924; Reid, 1890; Sordelli, 1882; Whitehead, 1920

Goecke, 1943, 1960a 
Table 2. (Continued)

\begin{tabular}{|c|c|c|c|}
\hline Taxon, author, year & Period & Location & Depositories, references, other \\
\hline D. sparganii Ahrens, 1810 & Quaternary & $\begin{array}{l}\text { Denmark and southern } \\
\text { Sweden }\end{array}$ & $\begin{array}{l}\text { Henriksen, 1914, 1933; Jessen, 1920, } \\
1923\end{array}$ \\
\hline $\begin{array}{l}\text { D. spinosa DeGeer (=? D. crassipes } \\
\text { Fabricius, 1792) }\end{array}$ & Quaternary & Schonen, Sweden & $\begin{array}{l}\text { Andersson, 1889; Henriksen, 1914, } \\
\text { 1933; Kurck, } 1917\end{array}$ \\
\hline $\begin{array}{l}\text { D. splendida Théobald (in Piton and } \\
\text { Théobald, 1935) (Fig. 57) }\end{array}$ & Miocene to Quaternary & $\begin{array}{l}\text { L'Auvergne, Masif Central, } \\
\text { France }\end{array}$ & $\begin{array}{l}\text { no. 14, 21, Coll. L. Piton; Goecke, } \\
\text { 1960a }\end{array}$ \\
\hline D. statzi Goecke, 1943 (Fig. 58) & Eocene & Germany & Goecke, 1960a \\
\hline D. stiria Scudder, 1890b (Fig. 59) & Quaternary & $\begin{array}{l}\text { Interglacial clays near Scar- } \\
\text { borough, Ontario and Leda, } \\
\text { Ottawa, Canada }\end{array}$ & $\begin{array}{l}\text { Goecke, 1943, 1960a; Scudder, 1890a, } \\
\text { b, 1892, 1895a; Wickham, } 1920\end{array}$ \\
\hline $\begin{array}{l}\text { D. stirioides Wickham, } 1917 \text { (also as } \\
\text { D. styrioides in same paper) }\end{array}$ & Pleistocene & $\begin{array}{l}\text { Sangamon peat, Sangamon } \\
\text { River, near Mahomet, } \\
\text { Champaign Co., Illinois, } \\
\text { USA }\end{array}$ & $\begin{array}{l}\text { National Museum of Natural History } \\
\text { (NMNH = USNM), Washington, D. } \\
\text { C., USA; Wickham, 1920; Goecke, } \\
\text { 1960a }\end{array}$ \\
\hline D. subtilis group & Quaternary & Innerkip, Ontario, Canada & $\begin{array}{l}\text { Quaternary Entomology Laboratory, } \\
\text { University of Waterloo Canada; } \\
\text { Pilny and Morgan, } 1987\end{array}$ \\
\hline $\begin{array}{l}\text { D. tenuipunctata Théobald (in Piton } \\
\text { and Théobald, 1935) (Fig. 60) }\end{array}$ & Miocene to Quaternary & $\begin{array}{l}\text { Cinérites du lac Chambon, } \\
\text { L'Auvergne, Masif Central, } \\
\text { France }\end{array}$ & $\begin{array}{l}\text { no. } 12 \text {, deposited at Collection } \\
\text { Théobald; Goecke, 1960a }\end{array}$ \\
\hline D. thalassina Germar, 1811 & Quaternary & $\begin{array}{l}\text { Wester Cumberland, England; } \\
\text { Hösbach in Bayern, Ger- } \\
\text { many; Denmark and south- } \\
\text { ern Sweden; Belorussia, } \\
\text { former Soviet Union }\end{array}$ & $\begin{array}{l}\text { some specimens probably at the } \\
\text { Paleontomological Institute of the } \\
\text { Academy of Sciences, Moscow; } \\
\text { Andersson, 1889; Flach, 1884; Hen- } \\
\text { riksen, 1933; Holst, 1908; Kurck, } \\
\text { 1917; Meunier, 1901; Nasarow, 1984; } \\
\text { Pearson, 1962 }\end{array}$ \\
\hline D. near thalassina Germar, 1811 & Quaternary & central and southern Finland & Poppius, 1911 \\
\hline D. thyphae Ahrens & Quaternary & south Schonen, Sweden & Andersson, 1889 \\
\hline $\begin{array}{l}\text { D. versicolorea Brahm, } 1790(=? D \text {. } \\
\text { bidens Olivier })\end{array}$ & Quaternary & $\begin{array}{l}\text { Denmark and southern } \\
\text { Sweden }\end{array}$ & Andersson, 1889; Henriksen, 1933 \\
\hline $\begin{array}{l}\text { D. vicina Haupt, } 1956(=\text { D. haupti } \\
\text { Goecke, 1959?) (Figs. 61-62) }\end{array}$ & Eocene & Geiseltal, Germany & $\begin{array}{l}\text { type, G 55/75, Sammlung des } \\
\text { Geologisch-Paläontologischen Insti- } \\
\text { tuts der Universität Halle, Germany; } \\
\text { Goecke, } 1960 \mathrm{a}\end{array}$ \\
\hline D. woigti Goecke, 1943 (Fig. 63) & Eocene & Geiseltal, Germany & Goecke, 1960a \\
\hline $\begin{array}{l}\text { D. vulgaris Zschach, } 1788 \quad(=? \quad D \\
\text { typhae })\end{array}$ & late Pliocene - Quaternary & $\begin{array}{l}\text { West Cumberland, England; } \\
\text { Dogger Bank, North Sea, } \\
\text { long. } 2-5^{\circ} \mathrm{E} \text {, lat. } 54-56^{\circ} \mathrm{N} \text {, } \\
\text { ca. } 200 \mathrm{~km} \text { NNE London; } \\
\text { Denmark and southern } \\
\text { Sweden }\end{array}$ & $\begin{array}{l}\text { Andersson, I889; henriksen, 1933; } \\
\text { Pearson, 1962; Whitehead, } 1920 \\
\text { Whitehead and Goodchild, 1909, fed } \\
\text { on Typha }\end{array}$ \\
\hline D. weigelti Goecke, 1943 (Fig. 64) & Eocene & Gieseltal, Germany & Goecke, $1960 \mathrm{a}$ \\
\hline D. weylandi Goecke, $1960 \mathrm{~b}$ & Oligocene & Rott, Germany & not found \\
\hline $\begin{array}{l}\text { D. (Donacia) wightoni Askevold, } \\
1990 \mathrm{a}\end{array}$ & Paleocene & $\begin{array}{l}\text { Paskapoo Formation, } \\
\text { Alberta, Canada }\end{array}$ & $\begin{array}{l}\text { University of Alberta Paleontological } \\
\text { Collec. 5566(407); oldest known fossil } \\
\text { donacine }\end{array}$ \\
\hline Donacia spp. & $\begin{array}{l}\text { lower Oligocene to Quater- } \\
\text { nary }\end{array}$ & $\begin{array}{l}\text { West Cumberland, Lan- } \\
\text { cashire, England; Mooren } \\
\text { von Nordost-Seeland and } \\
\text { interglacial of Denmark; La } \\
\text { Taphanel, Massif Central } \\
\text { and Interglacial, Lauenberg, }\end{array}$ & $\begin{array}{l}\text { specimens at Quaternary Entomology } \\
\text { Section, University of Birmingham, } \\
\text { England; others possibly at Institute of } \\
\text { Systematic; others Experimental Zool- } \\
\text { ogy, Polish Academy of Sciences, } \\
\text { Krakow; Coll. Berlin; and at the insti- }\end{array}$ \\
\hline
\end{tabular}


Table 2. (Continued)

\begin{tabular}{ll}
\hline Taxon, author, year & \multicolumn{1}{c}{ Location } \\
\hline & Plbe, France; Brackwasser- \\
toff, Nykerk, The Nether- & lands; Glacial Wanne-Eickel, \\
& Posen, Cottbus, Germany; \\
Baltic amber, Russia and \\
Poland; Dogger Bank, North \\
Sea, long, 2-5 E, lat. 54-56" \\
N, Poland; ca. 200 km NNE \\
London; Denmark and \\
southern Sweden; nordost \\
Seeland, Denmark; Schonen, \\
Sweden; Lake Oeningen and \\
interglacial, Gondiswill-Zell, \\
Switzerland, Villechétif and \\
Vanne, France; Re in Val \\
Vigezo, Italy; Donnaz, \\
Leffe, Val Gandino, older \\
formation of Schossnitz, \\
Oeningen, Sitzerland, and \\
Spitzbergen; many extant \\
species from multiple Euro- \\
pean localities; Nushagak \\
and Holitna lowlands, \\
southwest Alaska; Innerkip, \\
Ontario, Canada; Lamb \\
Spring site, Colorado; \\
Wedron, Illinois; Norwood, \\
south-central Minnesota; \\
Gervais Formation, Min- \\
nesota; Winter Gulf site, ca. \\
North Collins, New York; \\
Missouri Coteau, North \\
Dakota; Kewaunee, Wiscon- \\
sin, USA
\end{tabular}

Donacia sp., larvae and pupae (Figs. 65-66)

Donaciella cinerea herbst, $1784(=$ ? D. hydrocharidis Fabricius)

D. clavipes (Fabricius, 1792) (as Donacia clavipes) $(=$ ? Donacia menyanthidis Fabricius, 1792)
Eocene

Quaternary

Miocene - Quaternary

Quaternary Donacia tomentosa)

Eodonacia goeckei (Haupt, 1956) (Eodonacia $=$ D. (Donacia), Askevold, 1991) (Figs. 67, 68)
Depositories, references, other

tute of Arctic and Alpine Research, Boulder, CO, USA; Ashworth, 1972b, 1980; Ashworth and Schwert, 1992; Ashworth and Brophy, 1972, 1981; Bell and Bell, 1873; Benassi, 1896; Budde, 1937; Elias and Nelson, 1989; Elias and Wilkinson, 1983; Fliche. 1876; Förster, 1891; Garry et al 1987; Garry et al., 1990 Goecke, 1943. Hartz, 1902, 1909; Hartz and Milthers, 1901; Heer, 1876; Helm, 1896; Henriksen, 1914; Hieke and Pietrzeniuk, 1984; Jensen, 1920; Jentzsch, 1910; Klebs, 1910; Kurck, 1910, 1917; Lyell, 1840; Meunier. 1900, 1901; Nehring, 1895; Pawlowski et al., 1987; Pearson, 1962; Pilny and Morgan, 1987; Ponel and Coope, 1990, Puni, 1881; Rostrup, 1859; Schwert and Morgan, 1980; Scudder, 1887; Short and Elias, 1987; Short et al., 1992; Spahr, 1981b; Studer, 1920-22; Westergard. 1912; Whitehead, 1920; in many fragments of elytra; some specimens at the Quaternary Entomology Laboratory, University of Waterloo, Canada Department of Biology, University of Wisconsin, River Falls, USA

Geiseltal, Germany

Haupt, 1956; "holotypus" G 55/83, 84 Sammlung des GeologischPaläontologischen Instituts der Universität Halle, Gcrmany

Denmark and southern Sweden; Switzerland

Possibly at Institute of Alpine and Arctic Research, Boulder, CO, USA; Elias and Wilkinson, 1983: Henriksen, 1933

some specimens probably at Pateontomological Institute of the Academy of Sciences, Moscow, others possibly at Institute of Systematic and Experimental Zoology, Polish Academy of Sciences, Krakow; others possible at Institute of Alpine and Arctic Research, Boulder, CO, USA; Kolbe. 1984; Henriksen, 1914; Jessen, 1923; Lesne, 1918a, b, 1925; Lortet and Chantre, 1872; Nasarow, 1984; Pawłowski et al., 1987; Pearson, 1962; Poppius, 1911; Whitehead, 1920; Whitehead and Goodchild, 1909

Denmark and southern Sweden; Poland?

some specimens possibly at Institute of Systematic and Experimental Zoology, Polish Academy of Sciences, Kraków; Henriksen, 1933; Pawlowski et al., 1987

Eocene Geiseltal, Germany appears very similar to the extant $D$. D. crassipes Fabricius; Goecke, 1960a 
Table 2. (Continued)

\begin{tabular}{clc}
\hline Taxon, author, year & Period & Location \\
\hline E. paludosa Haupt, 1956 (Fig. 69) & Eocene & Geiseltal, Germany
\end{tabular}

Haemonia sp.

Hemidonacia insolita Haupt, 1956 (similar to D. (Cyphogaster) provostii Fairmaire, 1885; Hemidonacia $=D$. (Cyphogaster), Askevold, 1991) (Fig. 70)

Hemidonacia sp.
Macroplea appendiculata (Panzer,
1794)
M. mutica (Fabricius, 1792) (as Dona-
cia mutica)

M. nigrirostris Kirby

Neohaemonia nigricornis (Kirby, 1837)

Plateumaris affinis Kirby, 1837 (not Kuntze, 1818) $(=$ ? $P$. abdominalis Olivier) (Fig. 71)

P. braccatta (Scopoli, 1772)

P. consimilis Schrank.

$P$. cf. chalcea Lacordaire

P. discolor (Panzer, 1795) $(=? \quad D$. commari Suffrian, $=$ ? $P$. geniculata Th.) (Figs. 72) late Eocene to early Oligo- Baltic amber, Russia and cene

Eocene

Geiseltal, Germany
Depositories, references, other

type, G 55/73, 74, Sammlung des Geologisch-Paläontologischen Instituts der Universität Halle, Germany; Goecke, 1960a

Berendt, 1845; Spahr, 1981b

type, G 55/82, Sammlung des Geologisch-Paläontologischen Instituts der Universität Halle, Germany; Goecke, 1960a

\begin{tabular}{cl} 
Eocene & \multicolumn{1}{c}{ Geiseltal, Germany } \\
Quaternary & La Taphanel, Massif Central, \\
& France \\
Quaternary & $\begin{array}{l}\text { Schonen, Toppeladugard, } \\
\text { Sweden }\end{array}$ \\
Quaternary & Innerkip, Ontario, Canada
\end{tabular}

Quaternary

Missouri Coteau, Missouri; peat, Norwood, south-central Minnesota

Quaternary

Quaternary

Quaternary

Germany; Poland?

Quaternary

Innerkip, Ontario, Canada

Miocene to Quaternary

Denmark and southern Sweden; Chambéry, France; pre and interglacial from Lauenberg, Elbe; Dürten and Utznach, Hösbach in Bayern, Germany; widespread in Finland; Switzerland; Glaciales Torflager

Innerkip, Ontario, Canada
Haupt, 1956

Ponel and Coope, 1990

Andersson, 1889; Holst, 1906; Kurck, 1917

Quaternary Entomology Laboratory, University of Waterloo Canada; Pilny and Morgan, 1987

Department of Gcology, North Dakota State University, Fargo, North Dakota, USA; Ashworth and Brophy, 1972; Ashworth et al., 1981; Schwert, 1992

Goecke, 1943; Hartz, 1909; Henriksen, 1933

some specimens probably at Palcontomological Institute of the Academy of Sciences, Moscow (13/351), others possibly at Institute of Systematic and Experimental Zoology, Polish Academy of Sciences, Kraków; Andersson, 1889; Hcim and Gams, 1918; Henriksen, 1933; Nasarow, 1984; Pawłowski et al., 1987; Ponel and Coope, 1990

some specimens possibly at Institute of Systematic and Experimental Zoology, Polish Academy of Sciences, Kraków; Beyle, 1913; Pawłowslo et al. 1987

Quaternary Entomology Laboratory, University of Waterloo Canada; Pilny and Morgan, 1987

possibly at Institute of Artic and Alpine Research. Boulder, CO, USA Askevold, 1990, Elias and Wilkinson, 1983; Flach, 1884; Fliche, 1876; Goecke, 1943; Harpe, 1877; Heer, $1865,1876,1883 \mathrm{~b}$; Henriksen, 1933; Kolbe, 1894; Lortet and Chantre, 1872; Meunier, 1900, 1901; Poppius, 1911; "the most abundant donaciine in Dürsten and Utznach"

Quaternary Entomology Laboratory, University of Waterloo Canada; Pilnyu and Morgan, 1987 
Table 2. (Continued)

\begin{tabular}{|c|c|c|c|}
\hline Taxon, author, year & Period & Location & Depositories, references, other \\
\hline $\begin{array}{l}\text { P. fallax Haupt, } 1956 \text { (not a donaci- } \\
\text { ine. Askevold, 1991) (Fig. 73) }\end{array}$ & Eocene & Geiseltal, Germany & $\begin{array}{l}\text { type, G 55/81, Sammlung des } \\
\text { Geologisch-Paläontologischen Insti- } \\
\text { tuts der Universität Halle, Germany; } \\
\text { Goecke, } 1960 \mathrm{a}\end{array}$ \\
\hline P. flavipes (Kirby) & Quaternary & $\begin{array}{l}\text { Saylorville, lowa, USA; } \\
\text { Innerkip, Ontario, Canada }\end{array}$ & $\begin{array}{l}\text { Quaternary Entomology Laboratory, } \\
\text { University of Waterloo Canada; } \\
\text { Pilny and Morgan, 1987; Schwert, } \\
1992\end{array}$ \\
\hline
\end{tabular}

P. fulvipes (Kirby)

P. germari (Mannerheim)

$P$. cf. metallica Ahrens

\section{P. micans}

$P$. nigra (Fabricius, 1792) (as $D$. nigra)

P. primaeva (Wickham, 1912) (Figs. 74,90 ) (as Donacia primaeva) (indistinguishable from males of extant $P$. nitida Germar)

$$
\text { P. pusilla group }
$$

P. rustica (Kunze)

$P$. sericea (Linne, 1758) (frequently, as Donacia sericea L. or $D$. serica) (Fig. 75) late Quaternary

Quaternary

Quaternary

Quaternary

Quaternary

Miocene-Oligocene

Lefthand Reservoir and Mount Ida Ridge Pond, Front Range, Colorado, USA

Boreal Montana, False Cougar Cave, USA; Innerkip, Ontario, Canada

southeast Alaska, USA, Innerkip, Ontario, Canada

Nordost Seeland, Denmark

Schonen, Sweden; Nordost Seeland, Denmark

Florissant, Colorado

Quaternary

Quaternary

Miocene to Holocene
Poland?

Nuyakuk, Nushagak, and Holitna lowlands, southwestern Alaska, USA; Innerkip, Ontario, Canada; Lamb Spring site, Colorado, USA

glaciers, Torf, Soignies, Belgium; interglacial of Jüland, Denmark; Wolvercote, Oxfordshire, Aston Mill, Worcestershire, Mundesley, Staffordshire, Lancashire, West Cumberland, England; Dogger Bank, North Sea, long. $2-5^{\circ} \mathrm{E}$, lat. $54-56^{\circ} \mathrm{N}$, ca. $200 \mathrm{~km}$ NNE London; Denmark and southern Sweden; charbons de Durnten, France; Schulau, Niederelbe, Dürten, Holstein, and Utznach, Hösbach in Bayern, Germany; Denmark and southern Sweden; interglacial, Widhaus, Kanton St. Gallen, Switzerland; Belorussia, former Soviet Union; Poland?
Elias, 1985

Quarternary Entomology Laboratory, University of Waterloo Canada; Elias, 1991; Pilny and Morgan, 1987

Quaternary Entomology Laboratory, University of Waterloo Canada; Pilny and Morgan, 1987

Henriksen, 1914; Jessen, 1920

Andersson, 1889; Jessen, 1920; Kurck, 1917

holotype, AMNH 39488B, 39488A; MCZ 2601-2603=Scudder Coll. $8853,10177,11989$, respectively, other specimens 2601-2603 MCZ (no. 8853, 10177, and 11989 in Scudder coll.); Askevold, 1990a; Goecke, 1943, 1960a; Wickham, 1914b, 1920

Possibly at Institute of Arctic and Alpine Research, Boulder, CO, USA; Quaternary Entomology Laboratory, University of Waterloo, Canada; Elias and Short, 1992; Pilny and Morgan, 1987; Short and Elias, 1987; Short et al., 1992

some specimens possibly at Institutc of Systematic and Experimental Zoology, Polish Academy of Sciences, Kraków; Pawlowski et al. 1987

some specimens probably at Paleontomological Institute of the Academy of Sciences, Moscow (10/297), others possibly at Institute of Systematic and Experimental Zoology, Polish Academy of Sciences, Kraków; Andersson, 1889; Ashworth, 1972a, b; Bell, 1888, Beyle, 1901, 1913, 1920, 1924, 1926, 1931; Blair, 1923-24; Flach, 1884; Goecke, 1943; Hartz, 1909; Heer, 1958b, 1876; Heim and Gams, 1918; Henriksen, 1933; Holst, 1908; Lapougc, 1902; Kolumbe and Beyle, 1938; Meunier, 1901; Nasarow, 1984: Pawłowski et al., 1987; Pearson, 1962; Reid, 1890; Scoltz, 1934; Whitehead, 1920; Whitehead, 1989 
Table 2. (Continued)

\begin{tabular}{l} 
Taxon, author, year \\
\hline Plateumaris spp. (Fig. 76) \\
\\
Crioceris margarum Oustalet, 1874 \\
(Fig. 80 ) \\
vetusta (Heer, 1872?) (as Lema \\
Crioceris spp. \\
Clateumaris sp. or Donacia sp. (Fig. \\
(Figs. 78,79, and 91 ) \\
Criocerina sp. \\
Zeugophorinae \\
Sominella reticulata (Gyllenhal, 1817 ) \\
(as D. reticulata) \\
Donaciinae spp.
\end{tabular}

Lema t. cyanella Linné

L. evanescens Wickham, 1910 (Fig. 92)

L. fortior Wickham, 1914 b (Fig. 83, 93) late Cenozoic

Italy

Quaternary

Tertiary

Miocene

late Eocene to early Oligocene

Tertiary to Oligocene

Miocene

late Eocene to early Oligocene

Quaternary

Miocene to Oligocene

late Oligocene
Location

Wedron, Illinois; Missouri Coteau, North Dakota; Norwood, south-central and Lake Agassiz, Minnesota; Kewaunce, Wisconsin; Winter Gulf site, ca. North Collins, New York, USA; Lancashire, England; South Siberia, former Soviet Union; Tokai and Mikawa districs, central Japan

Schulau, Niederelbe, Germany; Tokai and Mikawa districs, central Japan

possibly Quaternary

Poland?; Denmark and southern Sweden; Wedron, Illinois, USA

Baltic amber, Russia and Poland

Florissant, Colorado, USA

Baltic amber, Russia and Poland

Provence, France

Lake Oeningen, Switzerland

Baltic amber, Russia and Poland; and Aix, Provence, France

La Taphanel, Massif Central, France

Florissant, Colorado, USA
Depositories, references, other

Vouchers at Department of Biology, University of Wisconsin, River Falls, USA; specimens in the Quaternary Entomology Laboratory, University of Waterloo Canada; Quaternary Entomology Section, University of Birmingham, England; Natl. Sci. Museum (Tokyo, Japan) NSMPA12096, NSM-12097; Ashworth and Schwert, 1992; Ashworth et al., 1972, 1981; Fujiyama, 1980 Garry et al., 1987, 1990; Martynov, 1929; Pilny and Morgan, 1987; Schwert and Morgan, 1980

Natl. Sci. Museum (Tokyo, Japan) NSM-PA12090; Beyle, 1901, 1913, 1920, 1924, 1926, 1931; Fujiyama, 1980

Malfatti, 1881

some specimens possibly at Institute of Systematic and Experimental Zoology, Polish Academy of Sciences, Kraków; Department of Biology, University of Wisconsin, River Falls; Garry et al., 1987; Henriksen, 1933; Pawlowski et al., 1987

Coll. Berlin; Hieke and Pietrzeniuk, 1984

MCZ 2615-2619, and possibly 438, 8644; Scudder Coll. 4458, 7977, 9577, 11242, 11737; Wickham, 1913a, 1920

Spahr, 1981b

Meunier, 1884; Théobald, 1937

Heer, 1876, 1883

Burmeister, 1836; Klebs, 1910; Scudder, 1887; Spahr, 1981b

Polne and Coope, 1990

type at Peabody Museum, Yale University, cat. no. 15, MCZ 2604 2611 other exemplars: Scudder Coll. $811,897,1985,3593,4956,8693$, 8919, 9595; Wickham, 1920

type, MCZ 2612, other specimens Scudder Coll. 8116, perhaps also MCZ 2613 and 2614, Scudder 3375 and 7762 are also conspecific; Wickham, 1920 
Table 2. (Continued)

\begin{tabular}{|c|c|c|c|}
\hline Taxon, author, year & Period & Location & Depositories, references, other \\
\hline $\begin{array}{l}\text { L. lesquereuxi Wickham, 1914a (Fig. } \\
84 \text { ) }\end{array}$ & Miocene & Florissant, Colorado & Wickham Coll., possibly at UNSM \\
\hline L. lichensis Voet. & Holocene & $\begin{array}{l}\text { Belorussia, former Soviet } \\
\text { Union }\end{array}$ & $\begin{array}{l}\text { some specimens probably at the } \\
\text { Palcontomological Institute of the } \\
\text { Academy of Sciences, Moscow (6/89); } \\
\text { Nasarow, } 1984\end{array}$ \\
\hline $\begin{array}{l}\text { L. pervetusta Cockerell, } 1921 \text { (Fig. } \\
\text { 85) }\end{array}$ & Eocene & $\begin{array}{l}\text { Rocky Mountains, Colorado, } \\
\text { USA }\end{array}$ & holotype, USNM 66579 (USGS 1299) \\
\hline L. pulchella Förster, 1891 (Fig. 86) & not found & Alsace-Lorraine, France & not found \\
\hline
\end{tabular}

L. trilinea White

L. tumulata Heyden and Heyden, 1865 (Fig. 87)

Lema sp.

\section{Clytrinae}

Clytra carbonaria Heyden and Heyden, 1865 (Fig. 94)

C. greithiana (Heer, 1847 (as elolon(ha) (Fig. 97)

\section{C. hippocastani (as Melolontha)}

C. pandorae Heer, 1847 (Fig. 95)

Clytrina eocenica Piton, 1940 (Fig. 243)

Labidostomis pyrrha Heyden and Heyden, 1866 (Fig. 96)

Labidostomissp.

Saxinis regularis Scudder, 1898 (Figs. 98, 99)

Smaragdina? incerta Zhang, 1989 (Fig. 100)

\section{Cryptocephalinae}

Cryptocephalites auratus Haupt, 1956 (Fig. 101)

\section{C. bidens Thomson}

C. elongatus Haupt, 1956 (Fig. 102)

C. punctatus Scudder, 1892? (Fig. 103)

C. rufiger Haupt, 1956 (Fig. 104)

Cryptocephalus miocenus Wickham, 1913c (Fig. 105)

C. minusculus Piton, 1940 (Fig. 244) late Quaternary

Tertiary

Tertiary to Quaternary

Miocene
Tertiary
Quaternary
Tertiary
Eocene
not found
not found
Pleistocene
mid-Miocene

Salzhausen, Germany; Lake Oeningen, Switzerland

Nordost Seeland, Denmark

Lake Oeningen, Switzerland

Menat

(Puy-de-Dome),

France

Siebengebirge, Germany

\section{Rott, Germany}

Old Hampshire Co., Massachusetts, USA

Shanwang, Shandong, China

Eocene

Tertiary to Quaternary

Eocene

Tertiary

Eocene

Miocene

Florissant, Colorado, USA

Eocene

Similkameen, British Colum-

Geiseltal, Germany
Lake Oeningen, Germany

Elias et al., 1992

not found

Förster, 1885, 1889; Pcarson, 1962; Scudder, 1887; Spahr, 1981b

Scudder, 1887

not found

Jessen, 1920

Giebel, 1852

Coll. L. Piton, no. 843

not found

Scudder, 1887

Wickham, 1920

S 830019, possibly Linqu Paleontological Museum, Shandong Provicial Museum, China

holotype, G 55/95, 96, Sammlung des Geologisch-Paläontologischen Instituts der Universität Halle, Germany

Kurck, 1917; Malfatti, 1881

type, G 55/105

Handlirsch, 1910; Scudder, 1985b; Wickham, 1920

holotype, G 55/97, other specimens $G$ $55 / 99,55 / 100,55 / 102$, Sammlung des Geologisch-Paläontologischen Instituts der Universität Halle, Germany.

type in Wickham's collection

Coll. L. Piton, no. 980
Menat (Puy-de-Dome),
France 
Table 2. (Continued)

\begin{tabular}{|c|c|c|c|}
\hline Taxon, author, year & Period & Location & Depositories, references, other \\
\hline C. pitoni Théobald, 1937 & Oligocene & Kleinkembs, France & $\begin{array}{l}\text { R. 809, Coll. Mieg du Museum de } \\
\text { Bâle; Théobald, } 1937\end{array}$ \\
\hline $\begin{array}{l}\text { C. relictus Schlechtendal, } 1893 \text { (Fig. } \\
\text { 106) }\end{array}$ & not found & $\begin{array}{l}\text { Braunkolengebirge, Ger- } \\
\text { many }\end{array}$ & $\begin{array}{l}\text { Königlichen mineralogischen Museum, } \\
\text { Germany }\end{array}$ \\
\hline C. rugosus Haupt, 1956 (Fig. 107) & Eocene & Geiseltal, Germany & $\begin{array}{l}\text { holotype, G } 55 / 101 \text {, other specimens } \\
\text { G } 55 / 102,55 / 103,44 / 104 \text {, Sammlung } \\
\text { des Geologisch-Paläontologischen } \\
\text { Instituts der Universität Halle, } \\
\text { Germany }\end{array}$ \\
\hline C. sericeus Linné $(=? C$. bidens Th. $)$ & Quaternary & $\begin{array}{l}\text { Denmark and southern } \\
\text { Sweden }\end{array}$ & Henriksen, 1933 \\
\hline Cryptocephalus nr. sericea & not found & amber & Helm, 1896 \\
\hline C. vetustus Scudder, 1878 (Fig. 108) & Eocene & $\begin{array}{l}\text { Green River, Wyoming, } \\
\text { USA }\end{array}$ & $\begin{array}{l}\text { Specimens } 4003,4004,4039,4044 \text {; } \\
\text { Scudder, } 1890 \mathrm{~b}\end{array}$ \\
\hline Cryptocephalus spp. & late Eocene to Quaternary & $\begin{array}{l}\text { Baltic amber, Russia and } \\
\text { Poland; La Taphanel, Massif } \\
\text { Central, France }\end{array}$ & $\begin{array}{l}\text { Klebs, 1910; Menge, 1856; Polne and } \\
\text { Coope, 1990; Scudder, 1887; Spahr, } \\
\text { 1981b }\end{array}$ \\
\hline Pachybrachis of mitis Fall & Quaternary & $\begin{array}{l}\text { northern Chihuahuan desert, } \\
\text { Texas and New Mexico, } \\
\text { USA }\end{array}$ & $\begin{array}{l}\text { Institute of Arctic and Alpine } \\
\text { Research, Boulder, CO, USA; Elias, } \\
\text { 1992b; Elias and Van Devender, } 1992\end{array}$ \\
\hline Pachybrachis spp. & Quaternary & $\begin{array}{l}\text { Arizona, California and } \\
\text { Kewaunee, Wisconsin, USA; } \\
\text { Chile; north eastern Siberia, } \\
\text { former Soviet Union }\end{array}$ & $\begin{array}{l}\text { Quaternary Entomology Laboratory, } \\
\text { North Dakota State University, Fargo, } \\
\text { USA; probably at Paleontology Insti- } \\
\text { tute, Academy of Sciences, Moscow; } \\
\text { Ashworth and Hoganson, 1983; Garry } \\
\text { et al., 1990; Hall et al, 1988; Hogan- } \\
\text { son and Ashworth, 1992; Kiselev, } \\
1981\end{array}$ \\
\hline $\begin{array}{c}\text { Eumolpinae } \\
\text { Adoxus obscurus (Lindé) }\end{array}$ & Quaternary & $\begin{array}{l}\text { West Cumberland, England; } \\
\text { Switzerland }\end{array}$ & $\begin{array}{l}\text { possibly at Institute of Arctic } \\
\text { and Alpine Research, Boulder, } \\
\text { CO, USA; Elias and Wilkinson, } \\
\text { 1983; Pearson, } 1962\end{array}$ \\
\hline Bromius obscurus (Linné, 1758) & late Cenozoic & $\begin{array}{l}\text { north eastern Siberia, former } \\
\text { Soviet Union }\end{array}$ & $\begin{array}{l}\text { probably at the Paleontology Institute, } \\
\text { Academy of Sciences, Moscow; } \\
\text { Kiselev, } 1981\end{array}$ \\
\hline Chalcosycia spp. NEW RECORD & $\begin{array}{l}\text { lower Eocene to lower Oli- } \\
\text { gocene }\end{array}$ & Dominican Republic amber & identified by S. Clark and JASB \\
\hline Colaspis aetatis Wickham, 1911 & $\begin{array}{l}\text { late Eocene to early Oligo- } \\
\text { cene }\end{array}$ & Florissant, Colorado, USA & $\begin{array}{l}\text { type at University of Colorado, } \\
\text { Boulder no. 61; Wickham, } 1920\end{array}$ \\
\hline $\begin{array}{l}\text { C. diluvialis Wickham, 1914b (Figs. } \\
\text { 109, 116) }\end{array}$ & $\begin{array}{l}\text { late Eocene to early Oligo- } \\
\text { cene }\end{array}$ & Florissant, Colorado, USA & $\begin{array}{l}\text { type, MCZ } 2626=\text { Scudder Coll. } \\
6872 ; \text { Wickham, } 1920\end{array}$ \\
\hline C. luti Scudder, 1893 (Figs. 110, 117) & Tertiary & North America & Scudder, 1990; Wickham, 1920 \\
\hline $\begin{array}{l}\text { C. proserpina Wickham, 1914b (Figs. } \\
\text { 111, 118) }\end{array}$ & not found & not found & Wickham, 1920 \\
\hline Colasposoma metallica & Oligocene to Holocene & Europe & Zherikhin, 1970 \\
\hline Colasposoma sp. & $\begin{array}{l}\text { late Eocene to early Oligo- } \\
\text { cene }\end{array}$ & $\begin{array}{l}\text { Baltic amber, Russia and } \\
\text { Poland }\end{array}$ & $\begin{array}{l}\text { Scuder, 1887; Spahr, 1981b; Zaddach, } \\
1868\end{array}$ \\
\hline $\begin{array}{l}\text { Eoeumolpinus azureviridis Haupt, } \\
1950 \text { (Fig. 112) }\end{array}$ & Tertiary to middle Eocene & Geiseltal, Germany & specimen 3852 \\
\hline $\begin{array}{l}\text { Eumolpites liberatus Heer, } 1865 \text { (Fig. } \\
\text { 21) }\end{array}$ & Jurassic to Tertiary & Switzerland & Heer, 1872,1876 \\
\hline Eumolpus spp. & $\begin{array}{l}\text { late Eocene to early Oligo- } \\
\text { cene }\end{array}$ & $\begin{array}{l}\text { Baltic amber, Russia and } \\
\text { Poland }\end{array}$ & $\begin{array}{l}\text { Helm, 1896; Spahr, 1981b; Zhang, } \\
1989\end{array}$ \\
\hline
\end{tabular}


Table 2. (Continued)

\begin{tabular}{|c|c|c|c|}
\hline Taxon, author, year & Period & Location & Depositories, references, other \\
\hline Graphops of. wyomingensis Blake & late Quaternary & $\begin{array}{l}\text { Lake Isabelle Delta site Left- } \\
\text { hand Reservoir, Front Range, } \\
\text { and lake Emma Colorado, } \\
\text { USA }\end{array}$ & $\begin{array}{l}\text { specimens possibly of Institute of } \\
\text { Arctic and Alpine Research, } \\
\text { Boulder, CO, USA Elias, 1985, Elias } \\
\text { et al., } 1991\end{array}$ \\
\hline Graphops spp. & Quaternary & $\begin{array}{l}\text { Peary Land, Greenland; } \\
\text { Innerkip, Ontario, Canada; } \\
\text { Missouri Coteau, North } \\
\text { Dakota, USA }\end{array}$ & $\begin{array}{l}\text { Quaternary Entomology Laboratory, } \\
\text { University of Waterloo, Canada; } \\
\text { others in the Zoological Museum, } \\
\text { University of Copenhagen, Denmark; } \\
\text { Ashworth and Schwert, 1992; Böcher, } \\
\text { 1989; Pilny and Morgan, } 1987\end{array}$ \\
\hline
\end{tabular}

Metachroma forissantensis Wickham, 1912 (Fig. 113)

Nodostoma sp.

Pachnephorus sp. (as Pachnophorus)

Profidia nitida Gressitt, 1963 (Fig. 115)

Pseudocolaspis sp.

unidentified Eumolpinae

Chrysomelinae

Calligrapha dislocata Rogers

Chrysochloa sp. (valid name is Oreina) (Fig. 120)

Chrysolina aurichalcea

C. bungei Jacoby

C. cavigera Sahlbr.

C. perforata Gebl.

C. rufilabris Falb.

C. septentrionalis Dejean (or Men.?)

Chrysolina sp.

Chrysomela bipunctatus Linné (many of the species placed in this genus are currently placed in Chrysolina) lower Eocene to early Miocene

late Eocene to lower Oligocene

late Eocene to lower Oligocene

Miocene to Oligocene

late Eocene to lower Oligocene

Tertiary

late Quaternary

Oligocene to Quaternary

late Cenozoic

not found

late Cenozoic

late Cenozoic

late Cenozoic

late Cenozoic

Holocene

Quaternary
Florissant, Colorado, USA; Dominican Republic amber Poland

Baltic amber, Russia and Poland

Chiapas amber, México

Baltic amber, Russia and Poland

Baltic amber, Germany
Baltic amber, Russia and

type at University of Colorado, Boulder, USA; Wickham, 1920

Klebs, 1910; Spahr, 1981b

Klebs, 1910; Spahr, 1981b

University California, Berkeley, Museum Paleontology

Klebs, 1910; Spahr, 1981b

Coll. Berlin; Hieke and Pietrzeniuk, 1984

northern Chihuahuan desert, Texas and New Mexico, USA

Institute of Arctic and Alpine Research, Boulder, CO, USA; Elias, 1992b; Elias and Van Devender, 1992

West Cumberland, England; France

north eastern Siberia, former Soviet Union

not found

Belorussia and north eastern Siberia, former Soviet Union

north eastern Siberia, former Soviet Union

north eastern Siberia, former Soviet Union

north eastern Siberia and Belorussia, former Soviet Union

Nushagak Lowland southwestern Alaska; northern Chihuahuan desert, Texas and New Mexico, USA; Belorussia, former Soviet Union

West Cumberland, England
Pearson, 1962; Théobald, 1937

probably deposited at the Paleontology Institute, Academy of Sciences, Moscow; Kiselev, 1981

\section{not found}

probably deposited at the Paleontology Institute, Academy of Sciences, Moscow (16/433); Kiselev, 1981; Nasarow, 1984

probably deposited at the Paleontology Institute, Academy of Sciences, Mowcow; Kiselev, 198

probably deposited at the Paleontology Institute, Academy of Sciences, Moscow; Kiselev, 1981

probably at the Paleontology Institute, Academy of Sciences, Moscow (8/269); Kiselev, 1981; Nasarow, 1984

Institute of Arctic and Alpine Research, Boulder, CO, USA; probably deposited at Paleontomological Institute of the Academy of Sciences, Moscow; Elias and Van Devender, 1992; Lea et al., 1991; Nasarow, 1984

Pearson, 1962 
Table 2. (Continued)

\begin{tabular}{|c|c|c|}
\hline Taxon, author, year & Period & Location \\
\hline C. calami Heer, 1847 (Fig. 121) & Tertiary to late Cenozoic & $\begin{array}{l}\text { north eastern Siberia, former } \\
\text { Soviet Union }\end{array}$ \\
\hline C. cerealis (or like $C$. cerealis) & Quaternary & $\begin{array}{l}\text { La Taphanel, Massif Central, } \\
\text { France }\end{array}$ \\
\hline C. ceresti Théobald, 1937 (Fig. 122) & Oligocene & France \\
\hline C. debilis Oustalet, 1874 (Fig. 123) & Tertiary to Oligocene & Provence, France \\
\hline C. fastuosa Scopoli & Quaternary & $\begin{array}{l}\text { Denmark and southern } \\
\text { Sweden }\end{array}$ \\
\hline C. haemoptera (L.) & Quaternary & West Cumberland, England \\
\hline C. haydingeri Heer & not found & not found \\
\hline C. hilberi Lomnicki, 1894 (Fig. 124) & Pleistocene & not found \\
\hline $\begin{array}{l}\text { C. lichenis Richter, } 1820 \text { (valid name } \\
\text { is Chrysolina lichenis) (Fig. 125) }\end{array}$ & Pleistocene & not found \\
\hline C. lithographica Weyenbergh, $1869 \mathrm{a}$ & not found & not found \\
\hline $\begin{array}{l}\text { C. lyelliana Heer, } 1856 \mathrm{a} \text { (Fig. 126- } \\
\text { 127) }\end{array}$ & Tertiary to Oligocene & Provence, France \\
\hline C. mathrona Oustalet, 1874 (Fig. 128) & Tertiary & Provence, France \\
\hline $\begin{array}{l}\text { C. matheroni Oustalet, } 1874 \text { (Fig. } \\
\text { 129) }\end{array}$ & Oligocene & Provence, France \\
\hline C. punctigera Heer, 1847 (Fig. 130) & Tertiary & not found \\
\hline C. subsulcata Mnh. & not found & Lake Oeningen, Switzerland \\
\hline C. taimyrensis Medvedev & late Cenozoic & $\begin{array}{l}\text { north eastern Siberia, former } \\
\text { Soviet Union }\end{array}$ \\
\hline C. varians (Schall.) & Quaternary & West Cumberland, England \\
\hline $\begin{array}{l}\text { C. vesperalis Scudder, } 1893 \text { (also as } \\
\text { Chrysomelites vesperalis) (Figs. 131, } \\
\text { 136) }\end{array}$ & Miocene-Oligocene & Florissant, CO, USA \\
\hline $\begin{array}{l}\text { Chrysomela sp., including larvae (Fig. } \\
\text { 132) }\end{array}$ & Tertiary to Quaternary & $\begin{array}{l}\text { Peary Land, Greenland; } \\
\text { Oeningen, Switzerland; Aix, } \\
\text { Provence, France; Lexden } \\
\text { and Bielbecks, Eastern Nor- } \\
\text { folk, Yorkshire, Durdestone } \\
\text { Bay, England; Baltic amber, } \\
\text { Russia and Poland. Lamb } \\
\text { spring site, Colorado; } \\
\text { south central New Mexico, } \\
\text { USA }\end{array}$ \\
\hline
\end{tabular}

Chrysomelites alaskanus Heer, 1869 (Fig. 136)

C. allochlamys Cockerell, 1920b (Fig. 133)

C. azureus Haupt, 1956 (Fig. 134)
Tertiary

Eocene

Eocene
English Bay, Alaska

Bartonian, Bagshot Beds, Bournemouth, England

Geiseltal, Germany
Depositories, references, other

some specimens probably at the Paleontology Institute, Academy of Sciences, Moscow; Heer, 1872; Kiselev, 1981

Burmeister, 1832, 1836; Polne and Coope, 1990

holotype, F $73+68$, Coll. Fliche, Ecole des E. and F., Nancy, France; Théobald, 1937

Meunier, 1884; Théobald, 1937

Henriksen, 1933

Pearson, 1962

Oustalet, 1874

not found

Lomnicki, 1894

not found

Meunier, 1884; Oustalet, 1874; Théobald, 1937

Meunier, 1884; Oustalet, 1874

Meunier, 1884; Théobald, 1937

Giebel, 1852

Giebel, 1852; Heer, 1876, 1883a

probably at the Paleontology Institute, Academy of Sciences, Moscow; Kiselev, 1981

Pearson, 1962

$\mathrm{MCZ} 7851, \quad 10416,2627,2628$ (11264, 13649, Scudder Coll.); Scudder, 1893, 1900; Wickham 1914b, 1920

some specimens at Zoological Museum, University of Copenhagen, Denmark; others possibly at Institute of Arctic and Alpine Research, Boulder, CO, USA; Bell, 1888; Berendt, 1845; Böcher, 1989; Curtis, 1829; Elias, 1987; Elias and Nelson, 1989; Elias and Wilkinson, 1983; Giebel, 1856; Helm, 1896; Menge, 1856; Scudder, 1887; Spahr, 1981b; Westwood, 1854; most abundant chrysomelid genus in Baltic amber

Scudder, 1900; Wickham, 1920

British Museum 19008

type, G 55/132, Sammlung des Geologisch-Paläontologischen Instituts der Universität Halle, Germany 
Table 2. (Continued)

\begin{tabular}{l} 
Taxon, author, year \\
\hline C. bartonicus Cockerell, 1920b (Fig. \\
135) \\
C. bisornatus Haupt, 1956 (Fig. 139) \\
C. cupreus haupt, 1956 (Fig. 140) \\
C. danielis Cockerell, 1926 (Fig. 141) \\
C. fabricii Heer, 1868 (Fig. 142) \\
C. foveolatus Haupt, 1956 (Fig. 143) \\
C. lindhageni Heer, 1870 "nomen rel- \\
ictum" (Fig. 138 A-F, 144 ) \\
C. prodromus Heer, 1865 (Fig. 27) \\
C. quadrilineatus Cockerell, 1920b \\
(Fig. 145) \\
C. thulensis Heer, 1870 nomen relic- \\
tum (Fig. 150 A-D)
\end{tabular}

Chrysomelites spp.

Chrysothoracus tropicus Zhang, 1989 (Figs. 146-149)

Colaphellus alpinus Payskull

Crosita? sp.

Eochrysomela indecorata Haupt, 1956 (Fig. 151)

E. ornata Haupt, 1956 (Figs. 152153)

E. punctator Haupt, 1956 (Fig. 154)

E. pustulata Haupt, 1956 (Fig. 155)

Eomelasoma incostata Haupt, 1956 (Fig. 156)

\begin{tabular}{lc} 
Period & \multicolumn{1}{c}{ Location } \\
Eocene & $\begin{array}{l}\text { Barshot Beds, Bournemouth, } \\
\text { England } \\
\text { Gocene }\end{array}$ \\
Eocene & Geiseltal, Germany \\
Tertiary & $\begin{array}{l}\text { Sunchal, Provincia Jujuy } \\
\text { Argentina }\end{array}$ \\
Tertiary & $\quad$ Greenland
\end{tabular}

Tertiary to middle Eocene

\section{Greenland}

Geiseltal, Germany

Eocene

Jurassic to Tertiary

Eocene

Eocene

not found

mid-Miocene

late Cenozoic

late Cenozoic

Tertiary to middle Eocene

Tertiary to middle Eocene

Tertiary to middle Eocene

Tertiary to middle Eocene
Alaska; Spitzbergen, Greenland

Shanwang, Shandong, China

north eastern Siberia, former

Soviet Union

north eastern Siberia, former Soviet Union

Geiseltal, Germany

Geiseltal, Germany

Geiseltal, Germany

Geiseltal, Germany

Geiseltal, Germany
Depositories, references, other

British Museum, lost label, possibly 19022 , also marked with an " $x$ "

type, G 55/131, Sammlung des Geologisch-Paläontologischen Instituts der Universität Halle, Germany

type, G 55/133, Sammlung des Geologisch-Paläontologischen Instituts der Universität Halle, Germany

"will be placed in British Museum"

Heer, 1883a, b; Scudder, 1900; Wickham, 1920

type, G 55/129, 130, Sammlung des Geologisch-Paläontologischen Instituts der Universität Halle, Germany

"a small capsule or pod from some plant"; Wickham, 1920; apparently not Heer, 1883a, b; Scudder, 1900

Heer, 1865, 1872, 1876, 1879, 1883a; Scudder, 1885, 1887

British Museum, 19006

"some plant remains", one of fossil fragments like Metasequoia occidentalis (Newb.) (Taxodiaceae) seed fragment; Birket-Smith, 1977

Förster, 1889

S 82701, Shandong Provicial Museum, China

probably deposited at the Paleontology,

Academy of Sciences, Moscow; Kiselev, 1981

probably at the Paleontology Institute, Institute, Academy of Sciences, Moscow; Kiselev, 1981

type, G 55/113, other specimen, $55 / 114$; Sammlung des GeologischPaläontologischen Instituts der Universität Halle, Germany

type, G 55/111; Sammlung des Geologisch-Paläontologischen Instituts der Universität Halle, The Netherlands; apparently, described twice, with two specimens that look different (Haupt, 1956)

type, G 55/106, other specimens G 55/107-55/110; Sammlung des Geologisch-Paläontologischen Instituts der Universität Halle, Germany

holotype, G 55/112; Sammlung des Geologisch-Paläontologischen Instituts der Universität Halle, Germany

type, G 55/116; Sammlung des Geologisch-Paläontologischen Instituts der Universität Halle, Germany 
Table 2. (Continued)

\begin{tabular}{|c|c|c|c|}
\hline Taxon, author, year & Period & Location & Depositories, references, other \\
\hline E. scutellata Haupt, 1956 (Fig. 157) & Tertiary to middle Eocene & Geiseltal, Germany & $\begin{array}{l}\text { type, G 55/116; Sammlung des } \\
\text { Geologisch-Paläontologischen Insti- } \\
\text { tuts der Universität Halle, Germany }\end{array}$ \\
\hline Gastroidea polygoni (Linné) & Quaternary & West Cumberland, England & Pearson, 1962 \\
\hline Gastroidea sp. & Quaternary & West Cumberland, England & Pearson, 1962 \\
\hline $\begin{array}{l}\text { Gonioctena clymene Heer, } 1847 \text { (Fig. } \\
\text { 172) }\end{array}$ & Tertiary & Lake Oeningen, Switzerland & $\begin{array}{l}\text { Giebel, } 1852 \text {, Heer, } 1872 ; 1876 \text {; Ous- } \\
\text { talet, } 1874\end{array}$ \\
\hline G. japeti Heer, 1847 (Fig. 174) & Tertiary & Lake Oeningen, Switzerland & Giebel, 1852 \\
\hline $\begin{array}{l}\text { G. primordialis Assmann, } 1870 \text { (Fig. } \\
\text { 175) }\end{array}$ & Oligocene & $\begin{array}{l}\text { Schossnitz, Schlesien, Ger- } \\
\text { many }\end{array}$ & not found \\
\hline Gonioctena spp. & Holocene & $\begin{array}{lr}\text { Oeningen, } & \text { Switzerland; Aix, } \\
\text { Provence, } & \text { France; } \\
\text { Schossnitz, } & \text { Germany; } \\
\text { Belorussia, former Soviet } \\
\text { Union }\end{array}$ & $\begin{array}{l}\text { probably deposited at Palcontomologi- } \\
\text { cal Institute of the Academy of Sci- } \\
\text { ences, Moscow }(9 / 287) \text {; Nasarow, } \\
1984\end{array}$ \\
\hline $\begin{array}{l}\text { Gonocelis notatus Haupt, } 1950 \text { (Fig. } \\
\text { 158) }\end{array}$ & Tertiary to middle Eocene & Geiseltal, Germany & not found \\
\hline $\begin{array}{l}\text { Halocoleus cameratus Haupt, } 1950 \\
\text { (Fig. 159) }\end{array}$ & Tertiary to middle Eocene & Geiseltal, Germany & not found \\
\hline $\begin{array}{l}\text { Hemisphaerocosites sphaericus Haupt, } \\
1956 \text { (Fig. 160) }\end{array}$ & Tertiary to middle Eocene & Geiseltal, Germany & $\begin{array}{l}\text { type, G 55/115; Sammlung des } \\
\text { Geologisch-Paläontologischen Insti- } \\
\text { tuts der Universität Halle, Germany }\end{array}$ \\
\hline Hydrothassa glabra Herbst & Quaternary & $\begin{array}{l}\text { La Taphanel, Massif Central, } \\
\text { France }\end{array}$ & Polne and Coope, 1990 \\
\hline H. hannoverana Fabricius & late Cenozoic & $\begin{array}{l}\text { north eastern Siberia, former } \\
\text { Soviet Union }\end{array}$ & $\begin{array}{l}\text { probably at the Paleontology Institute, } \\
\text { Academy of Sciences, Moscow; } \\
\text { Kiselev, } 1981\end{array}$ \\
\hline Hydrothassa sp. & Pliocene-Quaternary & $\begin{array}{l}\text { Peary Land Greenland; } \\
\text { Switzerland }\end{array}$ & $\begin{array}{l}\text { probably at the Zoological Muscum, } \\
\text { University of Copenhagen, Denmark; } \\
\text { others possibly at Institute of } \\
\text { Arctic and Alpine Research, Boulder, } \\
\text { Co, USA; Böcher, 1989; Elias } \\
\text { and Wilkinson, } 1983\end{array}$ \\
\hline $\begin{array}{l}\text { Lina populeti Heer, } 1847 \text { (also as } L \text {. } \\
\text { populeti) (valid name is Chrysolina } \\
\text { populeti) (Fig. 176) }\end{array}$ & Tertiary & Lake Oeningen, Switzerland & $\begin{array}{l}\text { Heer, } 1858 \mathrm{~b}, 1872,1876,1883 \mathrm{a}, \mathrm{b} \text {; } \\
\text { Heyden, } 1862\end{array}$ \\
\hline $\begin{array}{l}L \text { sociata Heyden and Heyden, } 1866 \\
\text { (Fig. } 177 \text { ) }\end{array}$ & not found & Siebengebirge, Germany & not found \\
\hline $\begin{array}{l}L . \text { wetteravica Heyden } 1862 \text { (Fig. } \\
178 \text { ) }\end{array}$ & Tertiary & Salzhausen, Germany & not found \\
\hline Lina spp. & not found & $\begin{array}{l}\text { Oeningen, Switzerland; Rott, } \\
\text { Salzhausen, Germany }\end{array}$ & Scudder, 1887 \\
\hline $\begin{array}{l}\text { Melasoma aenea Linné (valid name is } \\
\text { Chrysomela) }\end{array}$ & Quaternary & southern Lappland, Finland & Poppius, 1911 \\
\hline $\begin{array}{l}\text { M. micropunctata Piton (in Piton and } \\
\text { Théobald, 1935) (valid name is Chry- } \\
\text { somela) (Fig. 181) }\end{array}$ & Quaternary & $\begin{array}{l}\text { Cinérites Varennes, } \\
\text { L'Auvergne, Masif Central, } \\
\text { France }\end{array}$ & $\begin{array}{l}\text { no. } 5 \text {, collection Station Limnologique } \\
\text { de Bessé }\end{array}$ \\
\hline $\begin{array}{l}\text { Melasoma sp. (valid name is Chry- } \\
\text { somela) }\end{array}$ & $\begin{array}{l}\text { late Eocene to early Oligo- } \\
\text { cene }\end{array}$ & $\begin{array}{l}\text { Baltic amber, Russia and } \\
\text { Poland }\end{array}$ & Spahr, $1981 b$ \\
\hline $\begin{array}{l}\text { Oreina amphyctionis Heer, } 1847 \text { (Fig. } \\
\text { 182) }\end{array}$ & Tertiary & Oeningen, Switzerland & not found \\
\hline O. hellenis Heer, 1847 (Fig. 183) & Tertiary & Oeningen, Switzerland & Giebel, 1852 \\
\hline O. protogeniae Heer, 1847 (Fig. 184) & Tertiary & Oeningen, Switzerland & Giebel, 1852 \\
\hline
\end{tabular}


Table 2, (Continued)

\begin{tabular}{|c|c|c|c|}
\hline Taxon, author, year & Period & Location & Depositories, references, other \\
\hline O. pulchra (Förster, 1891) (Fig. 185) & Oligocene & $\begin{array}{l}\text { Kleinkembs, Alsace- } \\
\text { Lorraine, France }\end{array}$ & $\begin{array}{l}\text { Specimen R 770, Coll. Mieg Museum } \\
\text { Bâle, France; Théobald, } 1937\end{array}$ \\
\hline Oreina spp. & Tertiary & peat, Lexden, England & Förster, 1886, 1889; Scudder, 1887 \\
\hline Phaedon armoraciae Thorell & Quaternary & $\begin{array}{l}\text { Denmark and southern } \\
\text { Sweden }\end{array}$ & Henriksen, 1933 \\
\hline P. cochleariae (Fabricius) & Holocene & $\begin{array}{l}\text { Aston Mill, Worcestershire, } \\
\text { England }\end{array}$ & Whitehead, 1989 \\
\hline P. t. tumidulum Germaı & Quaternary & $\begin{array}{l}\text { La Taphanel, Massif Central, } \\
\text { France }\end{array}$ & Polne and Coope, 1990 \\
\hline Phaedon spp. & late Cenozoic & $\begin{array}{l}\text { Lake Emma, Colorado, USA; } \\
\text { north eastern Siberia, former } \\
\text { Soviet Union }\end{array}$ & $\begin{array}{l}\text { specimens possibly at Institute of } \\
\text { Arctic and Alpine Research, } \\
\text { Boulder, CO, USA; and probably } \\
\text { at the Paleontology Institute, Academy } \\
\text { of Sciences, Moscow; Elias et al., } \\
\text { 1991; Kiselev, 1981 }\end{array}$ \\
\hline
\end{tabular}

Phratora vulgatissima (Linné) (valid name is Phyllodecta)

Phratora sp. (valid name is Phyllodecta)

Phyllodecta curtisii (Oustalet, 1874), as Gonioctena (valid name is Phratora) (Fig. 171)

P. vitellinae (Linné) (valid name is Phratora)

Phyllodecta sp. (valid name is Phratora)

Plagiodera lyelliana (Heer, 1956) $[=$ Chrysomela lyelliana Heer, 1856] $(=$ ? C. mathrona Oustalet, 1874)

P. novata (Heyden and Heyden, 1866) (Fig. 161)

Prasocuris aucta var. egena Zgl. (= Hydrothassa?)

Stenoplatys? fausti Weise

Strichosa eburata Blanch.

Timarcha metallica Laicharting,
1781-1784
Timarcha sp.

Zygogramma tortuosa Rogers

c.f. Zygospila sp.

Galerucinae

Adimonia? sp.
Quaternary

late Cenozoic

not found

Quaternary

Quaternary

Oligocene

not found

Pleistocene

late Cenozoic

Quaternary

Pleiostecene

late Eocene to Quaternary

late Quaternary

late Quaternary

Quaternary valley of the Seine, Paris

Belorussia and north eastern Siberia, former Soviet Union

Provence, France

Switzerland

La Taphanel, Massif Central, France

France

Rott, Siebengebirge, Germany

Hösbach, Bayern, Germany

north eastern Siberia, former Soviet Union

Chile

Höbach, Bayern, Germany

Baltic amber, Russia and Poland; Norfolk forest bed, England

northern Chihuahuan desert, Texas and New Mexico, USA

Arizona and California, USA
Lesne, 1925

probably at at the Palcontology Institute, Academy of Sciences, Moscow; Kiselev, 1981; Nasarow, 1984

Meunier, 1884; Théobald, 1937

possibly at Institute of Arctic and Alpine Research, Boulder, CO, USA; Elias and Wilkinson, 1983

Polne and Coope, 1990

specimen F. 141 Coll. Fliche, Ecole des E. and F., Nancy, France; Curtis, 1829; Meunier, 1884; Théobald, 1937

not found

Flach, 1884; Meunier, 1901

probably at the Paleontology Institute, Academy of Sciences, Moscow; Kiselev, 1981

at Quaternary Entomology Laboratory, North Dakota State University, Fargo, USA; Ashworth and Hoganson, 1983; Hoganson and Ashworth, 1992

Flach, 1884; Meunier, 1901

Bell, 1888; Spahr, 1981b

Institute of Arctic and Alpine Research, Boulder, CO, USA; Elias, 1992b; Elias and Van Devender, 1992

Hall et al., 1988 in peat at Jarville, Nancy, France
Fliche, 1875; Meunier, 1901; Scudder 1887 
Table 2. (Continued)

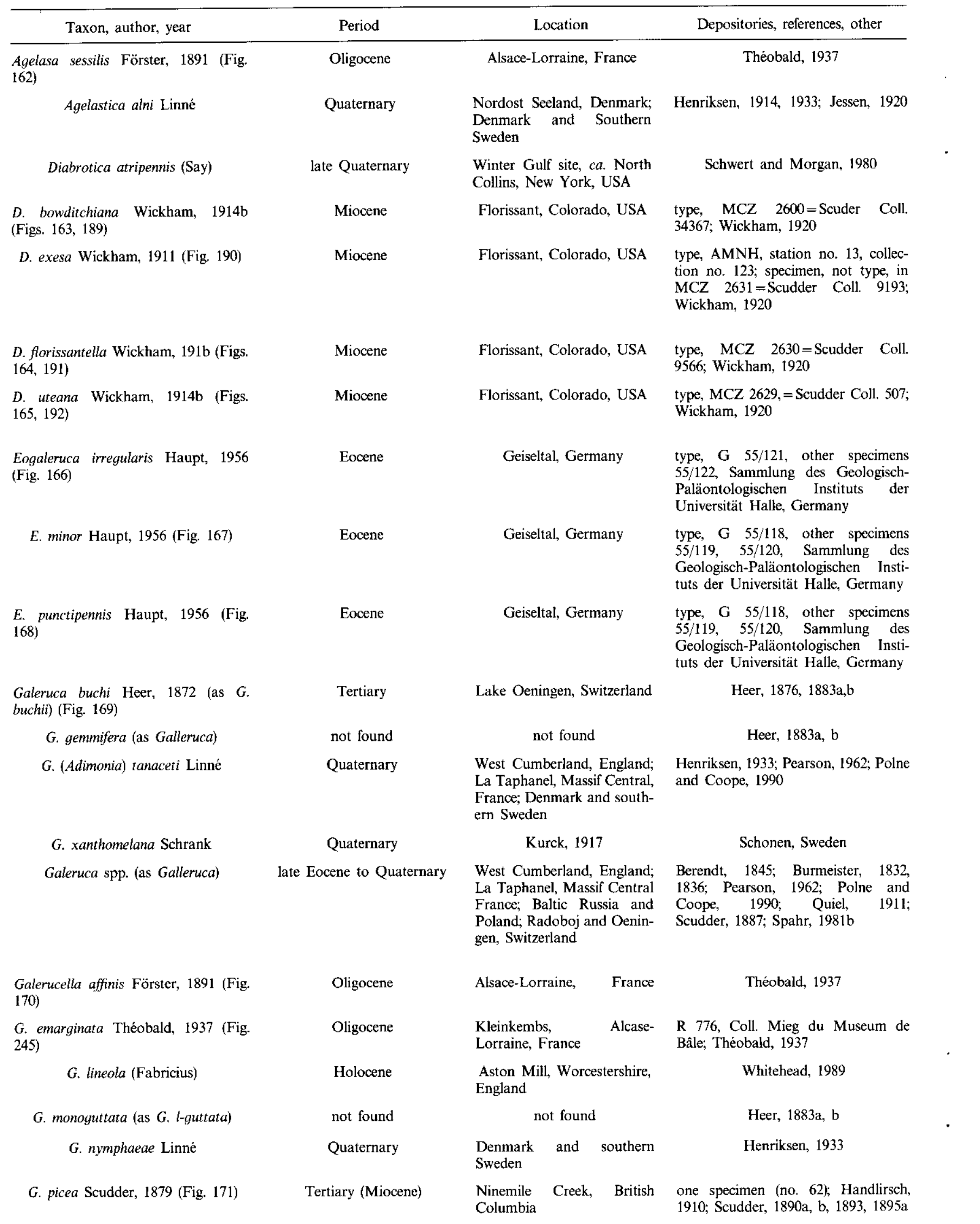


Table 2. (Continued)

\begin{tabular}{|c|c|c|c|}
\hline Taxon, author, year & Period & Location & Depositories, references, other \\
\hline Galerucella sp. & $\begin{array}{l}\text { late Eocene to lower Oligo- } \\
\text { cene }\end{array}$ & $\begin{array}{l}\text { Kleinkembs, France; Baltic } \\
\text { amber, Russia and Poland }\end{array}$ & $\begin{array}{l}\text { Klebs, 1910; Spahr, 1981b; Théobald, } \\
1937\end{array}$ \\
\hline $\begin{array}{l}\text { Hadroscelus schultzii Quedenfeldt, } \\
1885\end{array}$ & Cenozoic & Benguela, African copal & not found \\
\hline Hadroscelus sp. & not found & $\begin{array}{l}\text { Baltic amber, Russia and } \\
\text { Poland }\end{array}$ & Spahr, 1981b \\
\hline Leptonesiotes sp. NEW RECORD & $\begin{array}{l}\text { lower Eocene to lower Oli- } \\
\text { gocene }\end{array}$ & Dominican Republic amber & identified by $\mathrm{S}$. Clark \\
\hline Lochmaea capraea (Linné) & Quaternary & Switzerland & $\begin{array}{l}\text { possibly at Institute of Arctic and } \\
\text { Alpine Research, Boulder, CO, USA }\end{array}$ \\
\hline L. crategi Forster & Quaternary & $\begin{array}{l}\text { Nordost Seeland, Denmark; } \\
\text { Denmark and southern } \\
\text { Sweden }\end{array}$ & Henriksen, 1914, 1933; Jessen, 1920 \\
\hline $\begin{array}{l}\text { Luperodes submonillis } \text { Wickham, } \\
\text { 1914a (Fig. 179) }\end{array}$ & Miocene & Florissant, Colorado, USA & Wickham Coll.; Wickham, 1920 \\
\hline Luperus cf. flavipes (Linné) & Quaternary & West Cumberland, England & Pearson 1962 \\
\hline $\begin{array}{l}\text { L. fossilis Schlechtendal, } 1893 \\
\text { (Fig. 180) }\end{array}$ & not found & $\begin{array}{l}\text { Braunkohlengebirge of Rott, } \\
\text { Siebengebirge, Germany, }\end{array}$ & $\begin{array}{l}\text { Königlichen mineralogischen Muse- } \\
\text { ums }\end{array}$ \\
\hline Luperus sp. & late Eocene to late Cenozoic & $\begin{array}{l}\text { Baltic amber, Russia and; } \\
\text { Poland; north eastern Siberia, } \\
\text { former Sovict Union }\end{array}$ & $\begin{array}{l}\text { probably at the Paleontology Institute, } \\
\text { Academy of Sciences, Moscow; also } \\
\text { at Col. Berlin; Hieke and Pietrzeniuk, } \\
\text { 1984; Kiselev, 1981; Spahr, 1981b }\end{array}$ \\
\hline Monolepta sp. & $\begin{array}{l}\text { late Eocene to early Oligo- } \\
\text { cene }\end{array}$ & $\begin{array}{l}\text { Baltic amber, Russia and } \\
\text { Poland }\end{array}$ & Klebs, 1910; Spahr, 1981b \\
\hline Ophraella species A & late Quaternary & $\begin{array}{l}\text { Winter Gulf site, } c a \text {. North } \\
\text { Collins, New York, USA }\end{array}$ & Schwert and Morgan, 1980 \\
\hline $\begin{array}{l}\text { Pyrrhalta luteola } \quad \text { (Müller) }(= \\
\text { Galeruca luteola }=G . \quad \text { xanthomelaena } \\
\text { Schr.) }\end{array}$ & Quaternary & $\begin{array}{l}\text { Denmark and southern } \\
\text { Sweden }\end{array}$ & Henriksen, 1933 \\
\hline Pyrrhalta sp. & late Quaternary & $\begin{array}{l}\text { Umiakoviarusek, North- } \\
\text { eastern Labrador, Canada; } \\
\text { Lake Isabelle Delta site, } \\
\text { Front Range, Colorado; } \\
\text { Northern Chihuahuan desert, } \\
\text { Texas and New Mexico, USA }\end{array}$ & $\begin{array}{l}\text { Institute of Arctic and Alpine } \\
\text { Research, Boulder, CO, USA; Elias, } \\
1982, \quad 1985 \text {, Elias and Van } \\
\text { Devender, 1992; }\end{array}$ \\
\hline Scelolyperus sp. (as Scelyloperus) & Quaternary & $\begin{array}{l}\text { Big Bend Region, } \\
\text { Chihuahuan Desert, Texas; } \\
\text { south-central New Mexico, } \\
\text { USA }\end{array}$ & $\begin{array}{l}\text { Institute of Arctic and Alpine } \\
\text { Research, Boulder, CO, USA; Elias, } \\
\text { 1987; }\end{array}$ \\
\hline $\begin{array}{l}\text { Trirhabda majuscula Wickham, 1914b } \\
\text { (Figs. 186, 193) }\end{array}$ & Miocene & Florissant, Colorado, USA & $\begin{array}{l}\text { type, } 2634=\text { Scudder Coll. } 11266 ; \\
\text { Wickham, } 1920\end{array}$ \\
\hline $\begin{array}{l}T . \text { megacephala Wickham, } 1914 \mathrm{~b} \\
\text { (Figs. 187, 194) }\end{array}$ & Miocene & Florissant, Colorado, USA & $\begin{array}{l}\text { type, MCZ 2633, other specimen } 316 \text {; } \\
\text { Wickham, } 19206\end{array}$ \\
\hline $\begin{array}{l}\text { T. sepulta Wickham, } 1914 \text { b (Fig. } 188 \text {, } \\
\text { 195) }\end{array}$ & Miocene & Florissant, Colorado, USA & $\begin{array}{l}\text { type, MCZ } 2632=\text { Scudder Coll. } \\
\text { 3931; Wickham, } 1920\end{array}$ \\
\hline Trirhabda sp. & Quaternary & $\begin{array}{l}\text { south-central New Mexico, } \\
\text { USA }\end{array}$ & $\begin{array}{l}\text { Institue of Arctic and Alpine } \\
\text { Research, Boulder, CO, USA; Elias, } \\
1987\end{array}$ \\
\hline unidentified galerucines & Tertiary & $\begin{array}{l}\text { Baltic amber, Russia and } \\
\text { Poland }\end{array}$ & $\begin{array}{l}\text { Coll. Berlin; Hieke and Pietrzeniuk, } \\
1984\end{array}$ \\
\hline $\begin{array}{l}\text { Alticinae } \\
\text { Altica dryophyllorum Piton, 1940 (as } \\
\text { Haltica dryophyllorum) (Fig. 246) }\end{array}$ & Eocene & (Puy-de-Dome), & Coll. Piton, no. 447 \\
\hline
\end{tabular}


Table 2. (Continued)

\begin{tabular}{|c|c|c|c|}
\hline Taxon, author, year & Period & Location & Depositories, references, other \\
\hline A, dubia Förster, 1891 (Fig. 197) & Tertiary & Alsace-Lorraine, France & not found \\
\hline A. lythri (Aubè, 1843) (as H. lythri) & Holocene & $\begin{array}{l}\text { Aston Mill, Worcestershire, } \\
\text { West Cumberland, England }\end{array}$ & Pearson, 1962; Whitehead, 1989 \\
\hline A. magna Förster, 1891 (Fig. 198) & Oligocene & Alsace-Lorraine & Théobald, 1937 \\
\hline $\begin{array}{l}\text { A. oleracea (Linné, 1858) (as } H . \\
\text { oleracea) }\end{array}$ & Quaternary & widespread in Finland & Poppius, 1911 \\
\hline $\begin{array}{l}\text { A. quercetorum Foudras, } 1860 \text { (as } H \text {. } \\
\text { quercetorum) }\end{array}$ & Quaternary & $\begin{array}{l}\text { Denmark and southern } \\
\text { Sweden }\end{array}$ & Henriksen, 1933 \\
\hline $\begin{array}{l}\text { A. renovata Wickham, } 1914 \text { (as Hal- } \\
\text { tica renovata) (Figs. 199, 205) }\end{array}$ & Miocene & Florissant, Colorado, USA & $\begin{array}{l}\text { type at } \mathrm{MCZ} 2635=\text { Scudder Coll. } \\
7296 \text {, probably other specimens are } \\
\mathrm{MCZ} 2636,2637=\text { Scudder Coll. } \\
3507,7736 \text {, respectively; Wickham, } \\
1920\end{array}$ \\
\hline
\end{tabular}

Altica spp. (frequently as Haltica) late Eocene to Holocene Lancashire, West Cumberland, England; La Taphanel, Massif Central, France; Baltic amber, Russia and Poland; north eastern Siberia, former Soviet Union; Nushagak and Holitna Lowlands, southwest Alaska; Innerkip, Ontario, Canada; Lake Isabelle Delta site, Lake Isabelle peat site, Lefthand Reservoir, and Mount Ida Ridge Pond, Front Range Lake Emma, Lamb Spring Site, Mary Jane site, and Rocky Mountains National Park Colorado; Norwood, southcentral Minnesota; Wedron, Illinois; Big Bend Region, Chihuahuan Desert, Texas and New Mexico, USA; Chile

Apthona coerulera Payskull

A. puncticollis Piton, 1939 (Fig. 200) (homonym with A. puncticollis Allard, 1866)

Aphtona sp.

Apteropeda grossa Théobald (in Piton and Théobald, 1935) (as Apteropoda grossa) (Fig. 201)

Asiorestia antiqua (Gressitt, 1971) (Fig. 202) (as Crepidodera)

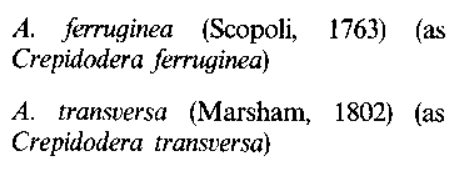

Asiorestia spp. (as Crepidodera)

\author{
Quaternary \\ Pliocène \\ West Cumberland, England \\ Lac Chambon, Puy-de- \\ Dôme, France
}

Quaternary

Quaternary

Miocene to Oligocene

Holocene
Holocene
Oligocene to Holo-

West Cumberland, England

Cinérites du lac Chambon, L'Auvergne, Masif Central, France

Chiapas, México

Aston Mill, Worcestershire, England

Aston Mill, Worcestershire, England

Baltic amber, Russia and Poland; Chile; Nushagak and Holitna lowlands, southwestern Alaska; Gervais Formation, Minnesota, USA
Quaternary Entomology Section, University of Birmingham, England; probably at the Paleontology Institute, Academy of Sciences, Moscow; Quaternary Entomology Laboratory, University of Waterloo, Canada; Quaternary Entomology Laboratory, North Dakota State University, Fargo, USA; Department of Biology, University of Wisconsin, River Falls, USA; Institute of Arctic and Alpine Research, Boulder, CO, USA; Ashworth, 1972b; Ashworth et al., 1972a, 1981; Ashowrth and Hoganson, 1983; Berendt, 1845; Burmeister, 1831, 1832, 1836; Elias, 1985; Elias and Nelson, 1989; Elias et al., 1986; 1991; Elias and Van Devender 1990, 1992; Förster, 1889; Helm, 1896, Garry et al, 1987; Hoganson and Ashworth, 1992; Kiselev, 1981; Pearson, 1962; Pilny and Morgan, 1987; Piton, 1940; Polne and Coope, 1990; Scudder, 1887; Short and Elias, 1987; Short et al., 1992; Spahr, 1981b;

\section{Pearson, 1962}

Coll. L. Piton, holotype, no. 1012

$$
\text { Pearson, } 1962
$$

no. 15 , collection $\mathrm{L}$. Piton

type at University of California, Berkeley, Museum of Palcontology 13523

Whitehead, 1989

Whitehead, 1989

Some at Quaternary Entomology Laboratory, North Dakota State University, Fargo, USA; Ashworth, 1980; Ashworth and Hoganson, 1983; Hoganson and Ashworth, 1992; Klebs, 1910; Leo et al. 1991; Short et al. 1992 
Table 2. (Continued)

\begin{tabular}{|c|c|c|}
\hline Taxon, author, year & Period & Location \\
\hline $\begin{array}{l}\text { Chaetocnema aridula Gyllenhal (C. a. } \\
\text { hortensis Geoffr.) }\end{array}$ & Holocene & $\begin{array}{l}\text { Belorussia, former Soviet } \\
\text { Union }\end{array}$ \\
\hline C. costulata (Motschulsky, 1860) & late Cenozoic & $\begin{array}{l}\text { north eastern Siberia, former } \\
\text { Soviet Union }\end{array}$ \\
\hline C. cribrata LeConte & late Quaternary & southwestern Alaska \\
\hline C. elongatula Hatch & late Quaternary & $\begin{array}{l}\text { northern Chihuahuan desert, } \\
\text { Texas and New Mexico, } \\
\text { USA }\end{array}$ \\
\hline C. obesa Boield. & Quaternary & $\begin{array}{l}\text { La Taphanel, Massif Central, } \\
\text { France }\end{array}$ \\
\hline Chaetocnema spp. & Quaternary & $\begin{array}{l}\text { Lancashire, England; La } \\
\text { Taphanel, Massif Central, } \\
\text { France; Switzerland Inner- } \\
\text { kip Ontario, Canada; Bida } \\
\text { Cave, Colorado Plateau, } \\
\text { Colorado; Winter Gulf site, } \\
\text { ca. North Collins, New York, } \\
\text { USA }\end{array}$ \\
\hline
\end{tabular}

Docemina crassipes Champion, 1918 (as Docemines, probably a typographic error)

Hippuriphila canadensis Brown

Longitarsus sp.

Mantura sp.

Ochrosis sp.

Oedionychus sp. (as Oedionychis)

Oryctocirtites protogaetm Scudder, 1876 (Figs. 203, 207)

Plectrotetrophanes hageni Wickham, 1914b (Figs. 204, 208)

Prochaetocnema florissantella Wickham, 1914b (Figs. 114, 119)

Psylliodes defguratus Théobald, 1937 (Fig. 247)

P. difficilis (Förster, 1891) (as $\mathrm{Hal}$ tica) (Fig. 196)

P. picina (Marsham, 1802)

P. polonica Lomnicki, 1894

Psylliodes sp.
Late Quaternary

late Quaternary

Quaternary

Quaternary

lower Oligocene

Quaternary

Miocene to Oligocene

Miocene

Miocene to Oligocene

Oligocene

Oligocene

Holocene

not found

Quaternary
Chile

Winter Gulf site, $c a$. North Collins, New York, USA

Denmark and southern Sweden

Lancashire, England

Baltic amber, Russa and Poland

Rocky Mountain National Park, CO, USA

Florissant, Colorado, USA

Florissant, Colorado, USA

Florissant, Colorado, USA

Kleinkembs, France

Brunnstatt and Klenkembs, Alsace-Lorraine, France

Aston Mill, Worcestershire, England

not found

La Taphanel, Massif Central, France
Depositories, references, other

probably deposited at Paleontomological Institute of the Academy of Sciences, Moscow; Nasarow, 1984

probably at the Palcontology Institute, Academy of Sciences, Moscow; Kiselev, 1981

Elias, 1992a

Institute of Arctic anc Alpine Research, Boulder, CO, USA; Elias, 1992b; Elias and Van Devender, 1992

Polne and Coope, 1990

Quaternary Entomology Section, University of Birmingham, England; Quaternary Entomology Laboratory, University of Waterloo, Canada; possibly at Institute of Arctic and Alpine Research, Boulder, CO, USA; Ashworth, 1972b; Elias et al., 1992; Elias and Wilkinson, 1983; Pilny and Morgan, 1987; Polne and Coope, 1990; Schwert and Morgan, 1980

Quaternary Entomology Laboratory, North Dakota State University, Fargo, USA Ashworth et al., 1991

Schwert and Morgan, 1980

Henriksen, 1933

Quaternary Entomology Section, University of Birmingham, England; Ashworth, 1972b

Klebs, 1910; Spahr, 1981b

possibly at Institute of Arctic and Alpine Research, Boulder, CO, USA; Elias et al. 1986

Wickham, 1920; Scudder, 1990

type, MCZ $2642=$ Scudder Coll. 8125; Wickham, 1920

type, MCZ $2643=$ Scudder Coll. 9430; Wickham, 1920

R 138, Coll. Mieg du Museum de Bâle; Théobald, 1937

R9 $+283,288+788,549$, and 106, Coll. Mieg du Museum de Bâle; Théobald, 1937

Whitehead, 1989

not found

Polne and Coope, 1990 
Table 2. (Continued)

\begin{tabular}{|c|c|c|c|}
\hline Taxon, author, year & Period & Location & Depositories, references, other \\
\hline $\begin{array}{l}\text { Systena florissantensis Wickham, } \\
1913 \text { b (Figs. 205, 209, and 210) }\end{array}$ & Miocene & Florissant, Colorado, USA & $\begin{array}{l}\text { type, USNM 59660; other specimens } \\
\text { at MCZ 2638-2641 = Scudder Coll. } \\
413,3430,8933,9615 \text {, respectively; } \\
\text { Wickham, } 1914 \mathrm{~b}, 1920\end{array}$ \\
\hline Walterianella sp. NEW RECORD & $\begin{array}{l}\text { lower Eocene to lower Oli- } \\
\text { gocene }\end{array}$ & Dominican Republic amber & identified by JASB and S. Clark \\
\hline Alticinae spp. & Tertiary to Quaternary & $\begin{array}{l}\text { Baltic amber, Russia and } \\
\text { Poland; Chile }\end{array}$ & $\begin{array}{l}\text { Coll. Berlin; Quaternary Entomology } \\
\text { Laboratory, North Dakota State } \\
\text { University, Fargo, USA; Ashworth } \\
\text { and Hoganson, 1983; Hieke and } \\
\text { Pietrzeniuk, 1984; Hoganson and Ash- } \\
\text { worth, } 1992\end{array}$ \\
\hline
\end{tabular}

\begin{abstract}
Hispinae
Anisodera sp.

"Anoplitis" bremii Heer, 1847 probably belong in another genus (Figs. 211-212)

Anoplitis sp. (Anoplitis is a synonym of Chalepus, many species of Anoplitis belong in Sumitrosis. Both genera are restricted to the New World.)

Chalepus americanus (Wickham, 1914b) (as Odontota americana Wickham, 1914b) (Figs. 213, 238)
\end{abstract}

Chalepus spp.

Dicladispa beskonakensis Nel, 1988 (Figs. 214-216)

D. muratensis Nel, 1988 (Figs. 217219)

Electrolema baltica Schaufuss, 1892 (Fig. 82)

\section{Hispa sp.}

ca. Microrhopala sp.

Odontota sp.

Oposispa scheelie Uhmann, 1939 (Fig. 220)

Oposispa sp.

Protanisodera glaesi Quiel, 1909

Protanisodera sp.

Sucinagonia javetana Uhmann, 1939 (Fig. 221)

Sucinagonia sp.

Hispinae spp.

$\begin{array}{ll}\begin{array}{l}\text { late Eocene to early Oligo- } \\ \text { cene }\end{array} & \begin{array}{l}\text { Baltic amber, Russia and } \\ \text { Poland } \\ \text { Lake Oeningen, Switzerland }\end{array} \\ \text { Tertiary } & \begin{array}{l}\text { Norwood, south-central Min- } \\ \text { nesota }\end{array}\end{array}$

late Oligocene to Miocene

late Eocene to early Oligocene

\section{Miocene}

Pliocene

late Eocene to early Oligocene

\section{lower Oligocene}

Tertiary to Holocene

$$
\text { not found }
$$

late Eocene to early Oligocene

\section{Oligocene}

late Eocene to early Oligocene

late Eocene to early Oligocene

late Eocene to early Oligocene

Oligocene

lower Oligocene to Holocene
Florissant, Colorado, USA

Baltic amber, Russia and Poland

Anatolia, Turkie

Murat, Cantal, France

Baltic amber, Russia and Poland

Baltic amber, Russia and Poland

Vancouver Island, Canada; Greenland

$$
\text { in amber }
$$

Baltic amber, Russia and Poland

\section{Europe}

Baltic amber, Russia and Poland

Baltic amber, Russia and Poland

Baltic amber, Russia and Poland

$$
\text { Europe }
$$

Baltic amber, Russia and Poland Lake Isabella Delta site, Front range, Colorado, USA
Spahr, 1981b

Giebel, 1852; Heer, 1847, 1872, 1876 , $1883 \mathrm{a}, \mathrm{b}$;

Ashworth et al., 1981

type, $\mathrm{MCZ} 2644=$ Scudder Coll. 7176 , another possible conspecific, MCZ $2645=$ Scudder Coll. 10506; Andersson, 1889; Wickham, 1920

Spahr, 1981b

IPMB-47738 Typothèque, Institut Paléontologie, Muséum National Histoire Naturelle, Paris

IPMR-07721 Typothèque, Institut Paléontologie, Muséum National Histoire Naturelle, Paris

Danzinger Provinzialmuseum, Germany; Korschefsky, 1939; Spahr, 1981b; Uhmann, 1939

Klebs, 1910; Spahr, 1981b

Chagnon, 1895; Handlirsch, 1910; Scudder, 1900; Zherikhin, 1970

Menge, 1856; Scudder, 1887

Larsson, 1978; Spahr, 1981b

Zherikhin, 1970

Museum für Naturkunde, Berlin; Spahr, 1981b

Spahr, 1981b

Larsson, 1978; Spahr, 1981b

Zherikhin, 1970

Col. Berlin; Elias, 1985; Hieke and Pietrzeniuk, 1984; Klebs, 1910 
Table 2. (Continued)

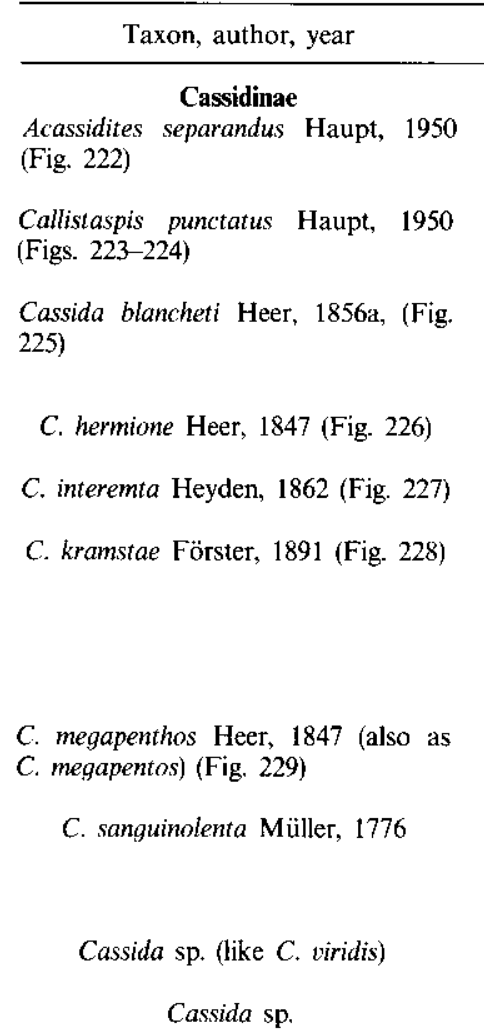

Coelocassida scabriusculum (Heer, 1870) (= Elytriditum scabriusculum Heer, 1870) (Fig. $239 \mathrm{~A}-\mathrm{H}$ )

\section{Delocrania sp}

Eocassida longula Haupt, 1950 (Fig. 230)

$$
\text { Inclusus sp. (Cassidinae?) }
$$

Mesomphalia gemmaspis Pongr., 1935 (Figs. 231-232)

Oligocassida melaena Théobald, 1937 (Fig. 248)

Paracassida aurichalcea Haupt, 1956 (Fig. 233)

P. bisangulata Haupt, 1956 (Fig. 234)

\author{
P. detrita Haupt, 1956 (Fig. 235)
}

P. punctillata Haupt, 1956 (Fig. 236)
Tertiary to middle Eocene

Tertiary to middle Eocene

Tertiary to Oligocene

$$
\text { Tertiary }
$$

Tertiary

Oligocene to late Cenozoic

$$
\text { Tertiary }
$$

late Cenozoic

not found

Eocene to Quaternary

\section{Eocene}

lower Eocene to lower Oligocene

Tertiary to middle Eocene

late Eocene to early Oligocene

Tertiary to middle Eocene

\section{Oligocene}

Tertiary to middle Eocene,

Tertiary to middle Eocene

Tertiary to middle Eocene

Tertiary to middle Eocene,
Geiseltal, Germany

Geiseltal, Germany

Geiseltal, Germany

Lake Oeningen, Switzerland; Provence, France

Lake Oeningen, Switzerland

Salzhausen, Germany

Riedisheim, Alsace-Lorraine, France; north eastern Siberia, former Soviet Union

Lake Oeningen, Switzerland

north eastern Siberia, former Soviet Union

not found

Denmark; Lexden, England; Baltic amber, Russia and Poland; Aix, Provence, France; Lexden, Oeningen, Switzerland; Rott, Germany; north eastern Siberia, former Soviet Union

Spitsbergen, Grönland

Dominican Republic amber

Geiseltal, Germany

Baltic amber, Russia and Poland

Geiseltal, Germany

Les Fumades, France

Geiseltal, Germany

Geiscltal, Germany

Geiseltal, Germany
Depositories, references, other

type 1526

specimens $1831,1711,2136,2165$

Heer, 1872, 1876; Meunier, 1884; Oustalet, 1874; Théobald, 1937

Giebel, 1852; Heer, 1872, 1876

not found

probably at the Paleontology Institute, Academy of Sciences, Moscow; also at Coll. Serv. carte géol., AlcascLorraine, France; Kiselev, 1981; Théobald, 1937

Giebel, 1852

probably at the Paleontology Institute, Academy of Sciences, Moscow; Kiselev, 1981

Burmeister, 1836

some probably at the Paleontology Institute, Academy of Sciences. Moscow; Bell, 1888; Curtis, 1829; Förster, 1885; Henriksen, 1922; Kiselev, 1981; Klebs, 1910; Scudder, 1887; Spahr, $1981 \mathrm{~b}$

not found

Farell, et al. 1992

not found

Spahr, $1981 b$

Haupt, 1950

Ni 18, Coll. Musée Nîmes.

type 55/128, Sammlung des Geologisch-Paläontologischen Instituts der Universität Halle, Germany

"holotypus G 55/125, 126", Sammlung des GeologischPaläontologischen Instituts der Universität Halle, Germany

type 55/127, Sammlung des Geologisch-Paläontologischen Instituts der Universität Halle, Germany

type, G 55/124, Sammlung des Geologisch-Paläontologischen Instituts der Universität Halle, Germany 
Table 2. (Continued)

\begin{tabular}{|c|c|c|c|c|}
\hline Taxon, author, year & & Period & Location & Depositories, references, other \\
\hline unidentified chrysomelids (Figs. 237) & $\begin{array}{l}\text { middle } \\
\text { nary }\end{array}$ & Miocene to Quater- & $\begin{array}{l}\text { Many localities in North } \\
\text { America and Europe; Peary } \\
\text { Land, Greenland; Nushagak } \\
\text { and Holitna lowlands, } \\
\text { southwestern Alaska; Tokai } \\
\text { and Mikawa districts, central } \\
\text { Japan; and in Shanwang, } \\
\text { Shandong, China }\end{array}$ & $\begin{array}{l}\text { S } 82749 \text { at Shandong Provincial } \\
\text { Museum, China, others at the Zoo- } \\
\text { logical Museum, University of } \\
\text { Copenhagen, Denmark; possibly at } \\
\text { Institute of Arctic and Alpine } \\
\text { Research, Boulder, CO, USA; Ash- } \\
\text { worth and Hoganson, 1983; } \\
\text { Bachofen-Echt, 1949; Böcher, 1989; } \\
\text { Elias, 1985; Elias et al., 1986, 1992; } \\
\text { Elias and Nelson, 1989; Elias and } \\
\text { Wilkinson, 1983; Fujiyama, 1980 } \\
\text { (Natl. Sci. Museum, Tokyo, Japan, } \\
\text { NSM-PA12084); Furth, 1978; Germar, } \\
\text { 1813; Giebel, 1856; Handlirsch, } \\
\text { 1906-1908a, 1906-1908b; Klebs, } \\
\text { 1910; Larsson, 1978; Lea et al., 1991; } \\
\text { Menge, 1856; Meunier, 1900; Quiel, } \\
\text { 1911; Scudder, 1887, 1891; Schaufuss, } \\
\text { 1892; Short et al., 1992 Uhmann, } \\
\text { 1939; Westwood, 1854, Zhang, 1989 }\end{array}$ \\
\hline
\end{tabular}




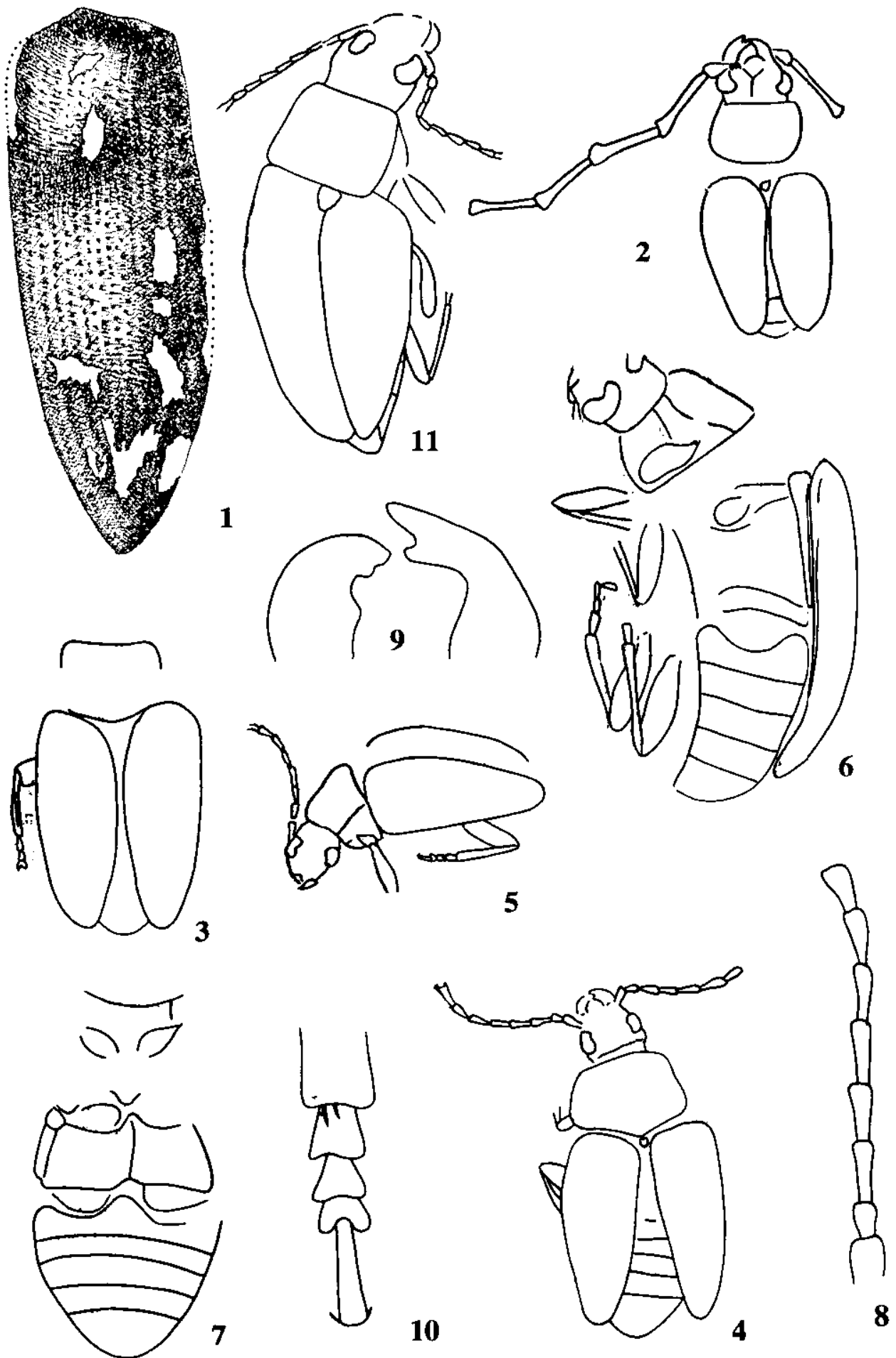

Plate 1. Fig. 1. Mesosagrites multipunctatus Martynov, 1935; Fig. 2. Cerambyomina longicornis Medvedev (in Rohdendorf, 1968 ); Figs. 3-10. Protoscelis jurassica Medvedev (in Rohdendorf, 1968). 3-4. Body, dorsal. 5. Dorsolateral. 6. Ventrolateral. 7. Ventral. 8. Antenna. 9. Mandibles. 10. Leg; Fig. 11. Protosceloides parvula Medvedev (in Rohdendorf, 1968). 

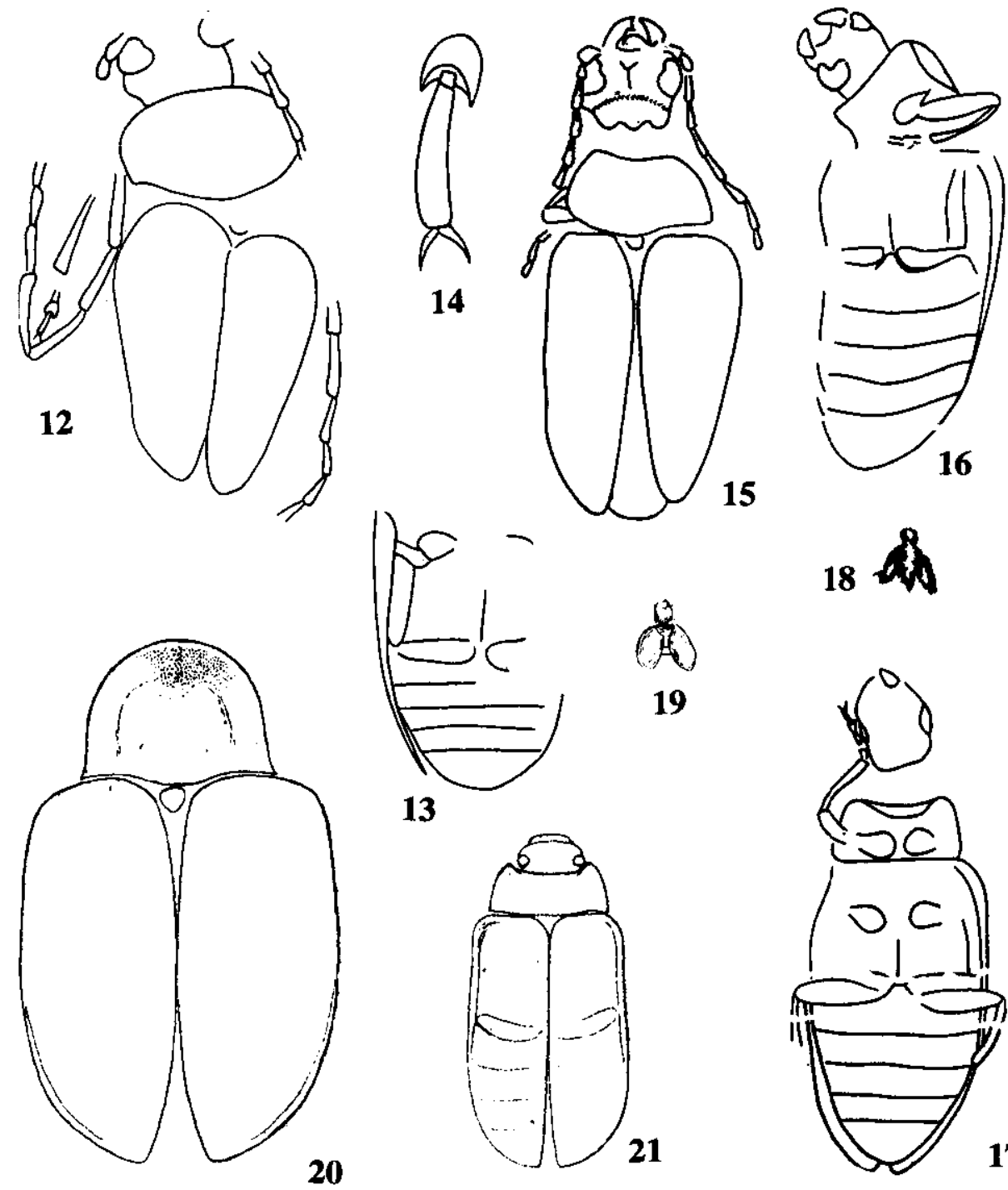

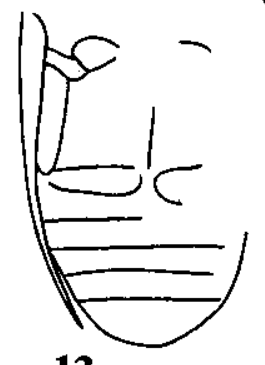

13

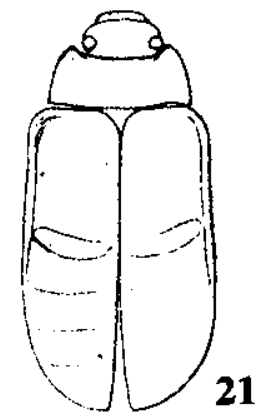

18

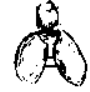

19

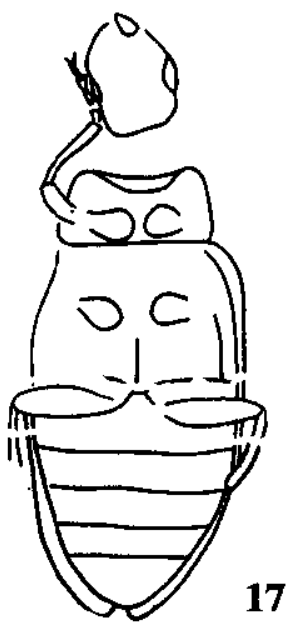

Plate 2. Figs. 12-14. Protosceloides nitidicornis Medvedev (in Rohdendorf, 1968). 12. Body, dorsal. 13. Abdomen, ventral. 14. Leg apex; Figs. 15-17. Pseudomegamerus grandis Medvedev (in Rohdendorf, 1968). 15. Dorsal. 16-17. Ventral; Figs. 18. Cryptocephalus antiquus Weyenbergh, 1869a; Figs. 19. C. mesozoicus Weyenbergh, 1869a. (From Oppenheim, 1887-1888); Fig. 20. Eumolpites jurassicus Martynov, 1926; Fig. 21. Eumolpites liberatus Heer, 1865 (From Heer). 


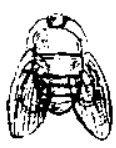

22

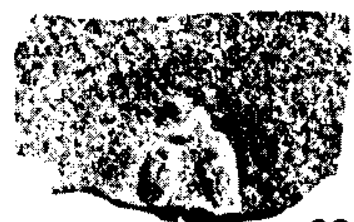

23
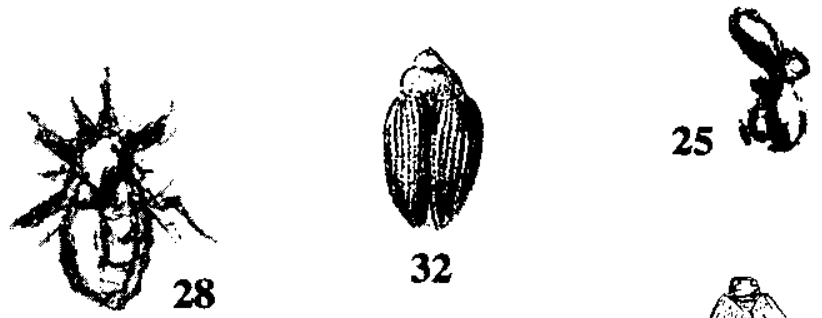

32

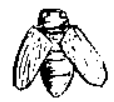

26
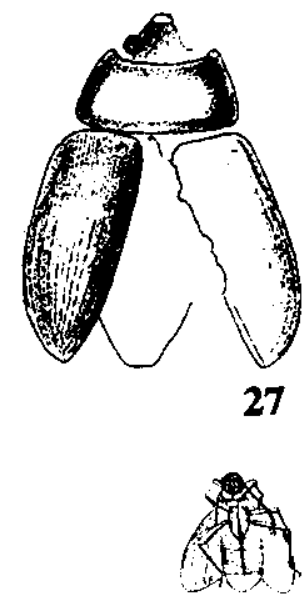

30
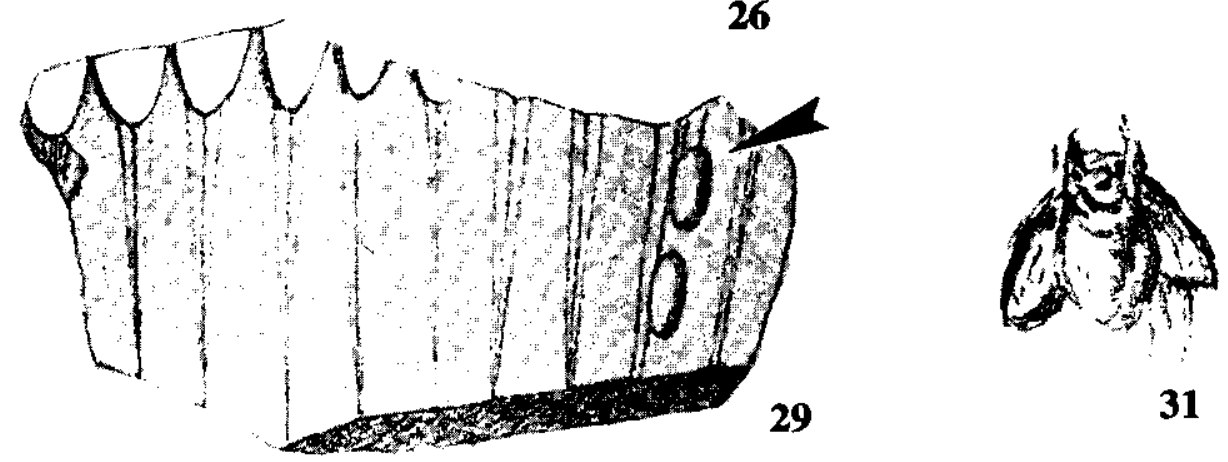

31

Plate 3. Figs. 22-23. Chrysomelites jurassicus Oppenheim, 1887-1888 (Fig. 23 from Meunier, 1898c); Figs. 24-25. Chrysomelites lithographica Weyenbergh, 1869a; Fig. 26. Chrysomelites minima Oppenheim, 1887-1888; Fig. 27. Chrysomelites prodromus Heer, 1865 ; Fig. 28. Chrysomelites rara Weyenbergh, 1869a; Fig. 29. C. rothenbachi Heer, 1877 (arrowhead) on Equisetum; Fig. 30. Galerucites carinata Oppenheim, 1887-1888. Note similarities with a heteropteran, such as a typical mirid; Fig. 31. Cassida aequivoca Weyenbergh, 1869 a: Fig. 32. Unidentified alleged chrysomelid. (From Brodie, 1845). 

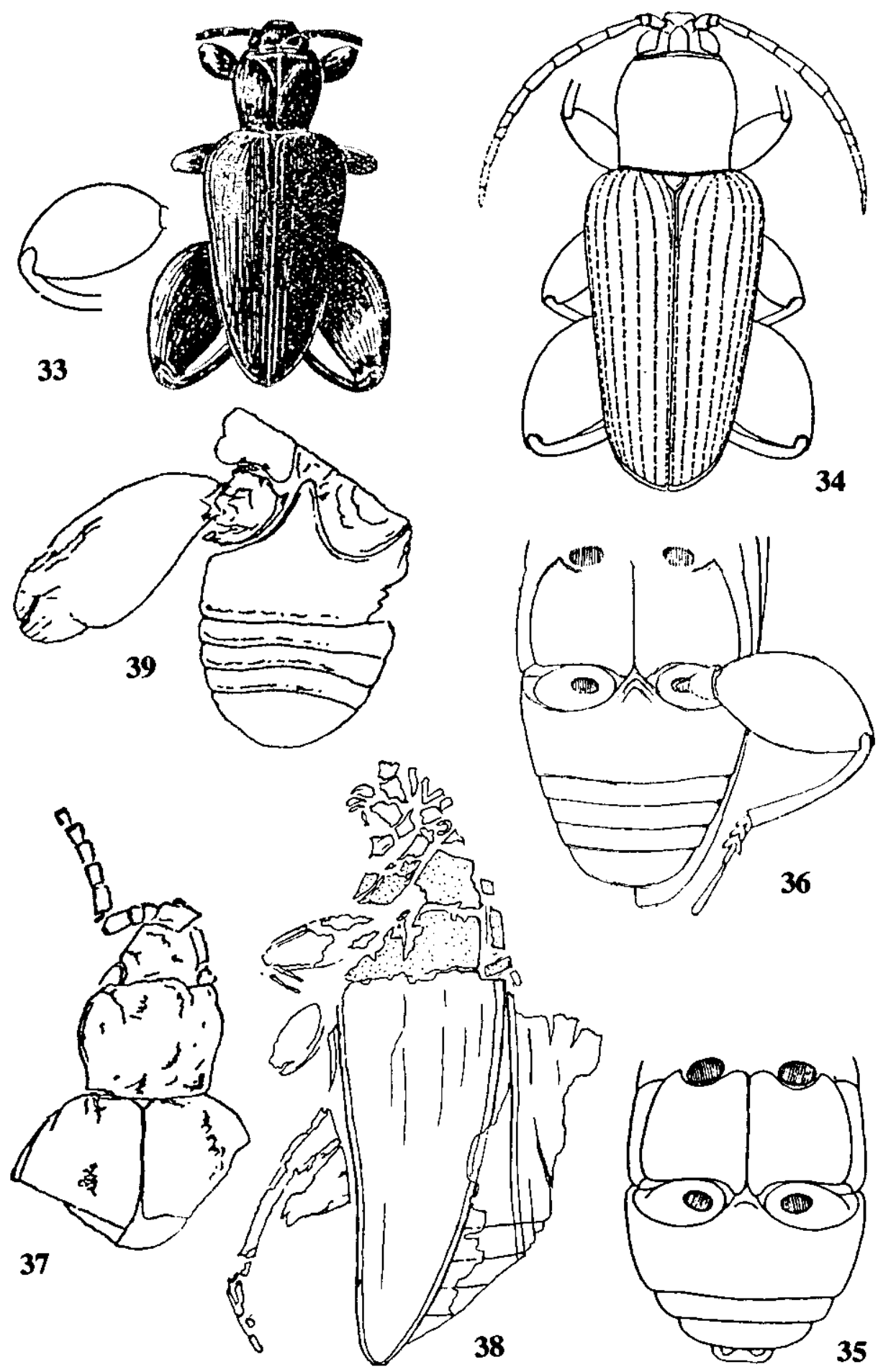

Plate 4. Fig. 33. Eosagra obliquata Haupt, 1950; Fig. 34-36. Eosagra subparallela Haupt, 1950. 34. Body, dorsal. 35 and 36 . Thorax and abdomen, ventral; Figs. 37-39. Eosagra sp. 37. Anterior part of body, dorsal. 38. Dorsolateral. 39. Thorax and abdomen, ventral. 


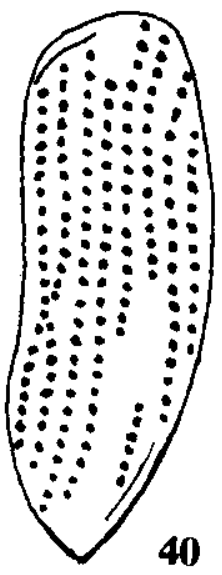

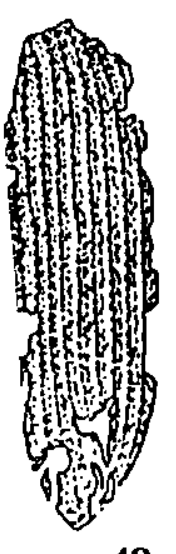

48

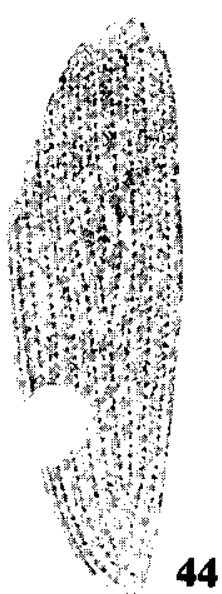

44

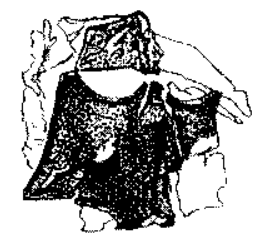

42
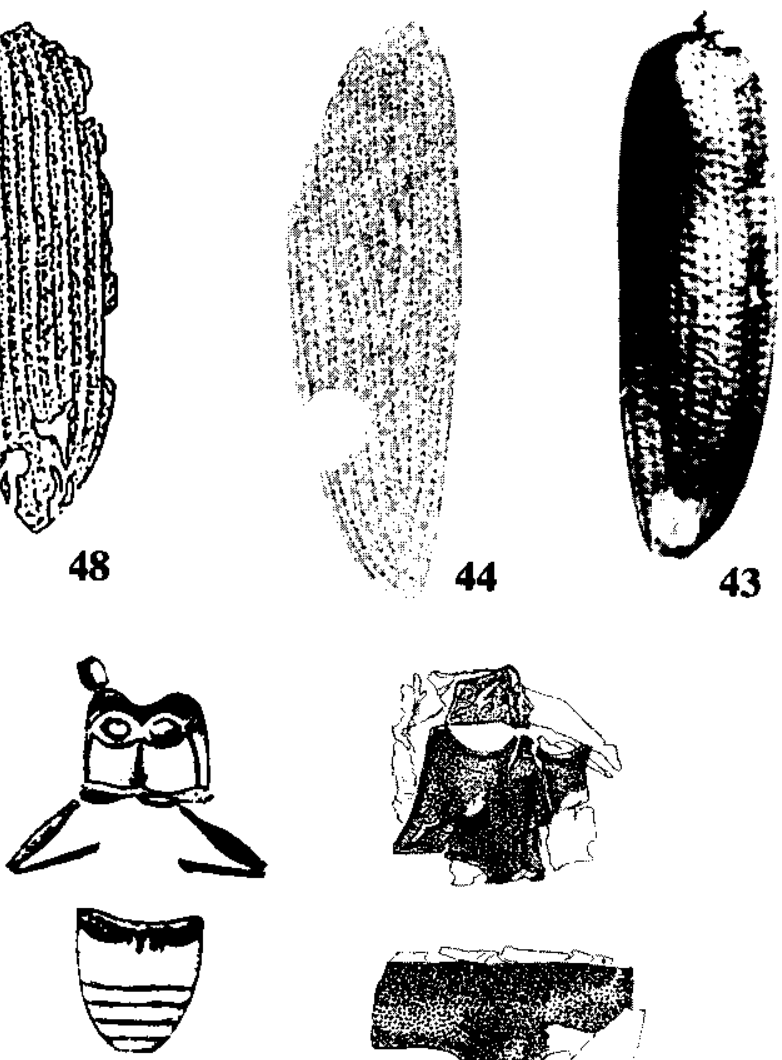

43
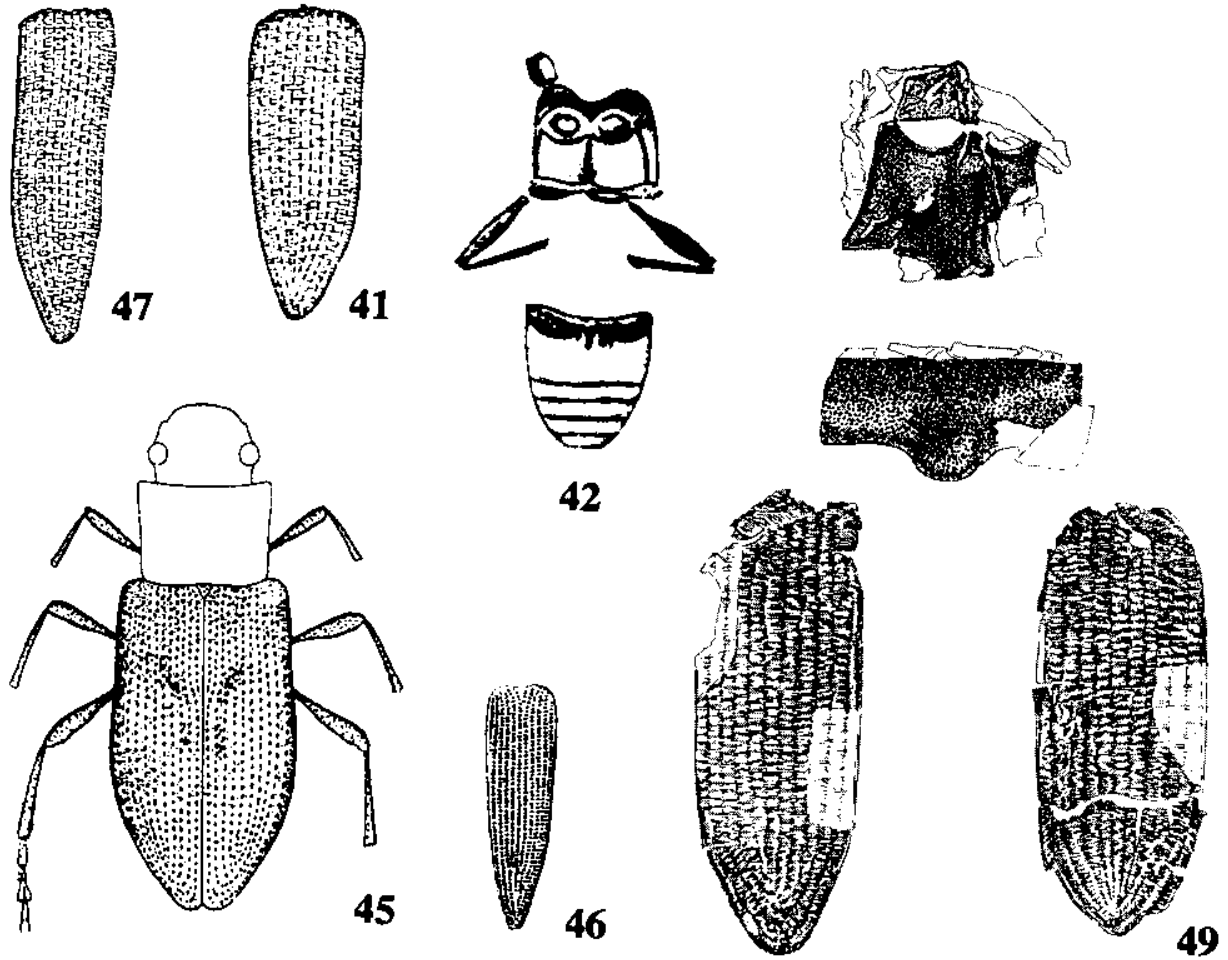

Plate 5. Fig. 40. Donacia pitoni Goecke, 1959? (=D. antiqua Piton, in Piton and Théobald, 1935); Fig. 41. D. bicolor Zschach, 1788 (From Goecke, 1943); Fig. 42. disjecta Förster, 1891 (From Elias, 1982); Fig. 43. D. distincta LeConte, 1850; Fig. 44. elongatula Scudder, 1990 (From Goecke, 1943); Fig. 45. D. jaroslavii Lomnicki, 1894; Fig. 46. D. lezneri Assmann, 1870; Fig. 47. D. lignitum Sordelli, 1882 (From Goecke, 1943); Fig. 38. D. minuta Haupt, 1956; Fig. 49. D. obscura Gyllenhal, 1813 (From Jessen, 1932). 

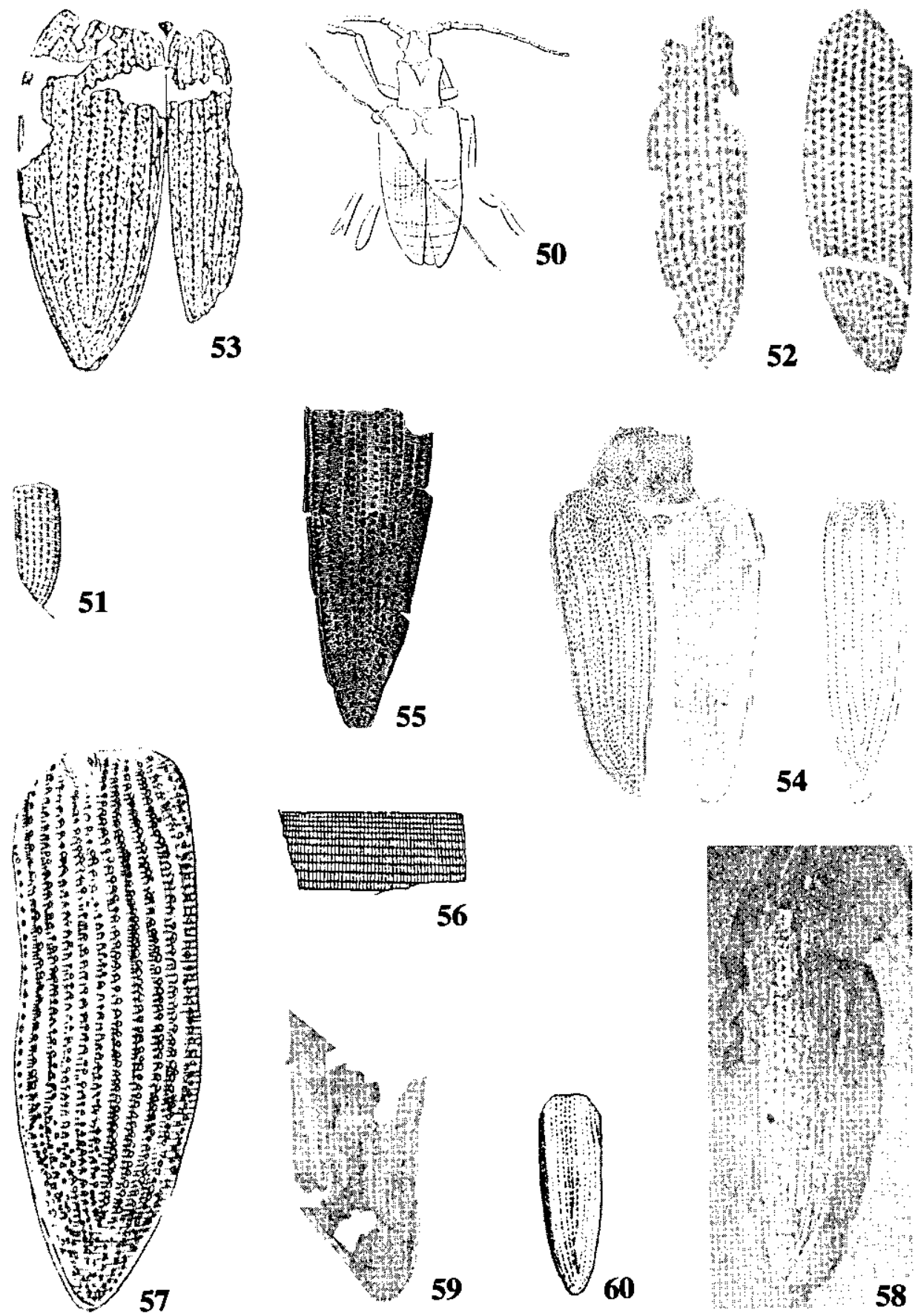

56
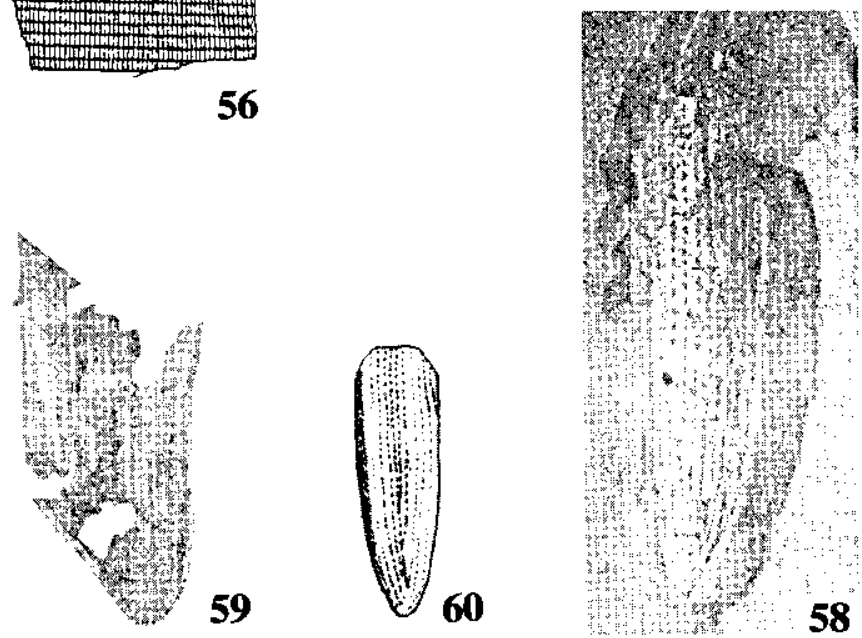

Plate 6. Fig. 50. Donacia palaemonis Heer, 1847; Fig. 51. D. parvula Heer, 1870 ('nomina relictum', Birket-Smith, 1977) (See also Figs. 88 A-D); Fig. 52. Elytra of D. pompatica Scudder, 1890b; Fig. 53. D. pterobrachys Haupt, 1956; Fig. 54. D. sagittariae Fabricius, 1792 (From Goecke, 1943); Fig. 55. D. semicuprea Panzer, 1796 (From Jessen, 1932); Fig. 56. D. smittiana Heer, 1870 ('nomina relictum', Birket-Smith, 1977) (See also Fig. 89); Fig. 57. D. splendida Théobald (in Piton and Théobald, 1935); Fig. 58. D. statzi Goecke, 1943; Fig. 59. D. stiria Scudder, 1890b; Fig. 60. D. tenuipunctata Théobald (in Piton and Théobald). 

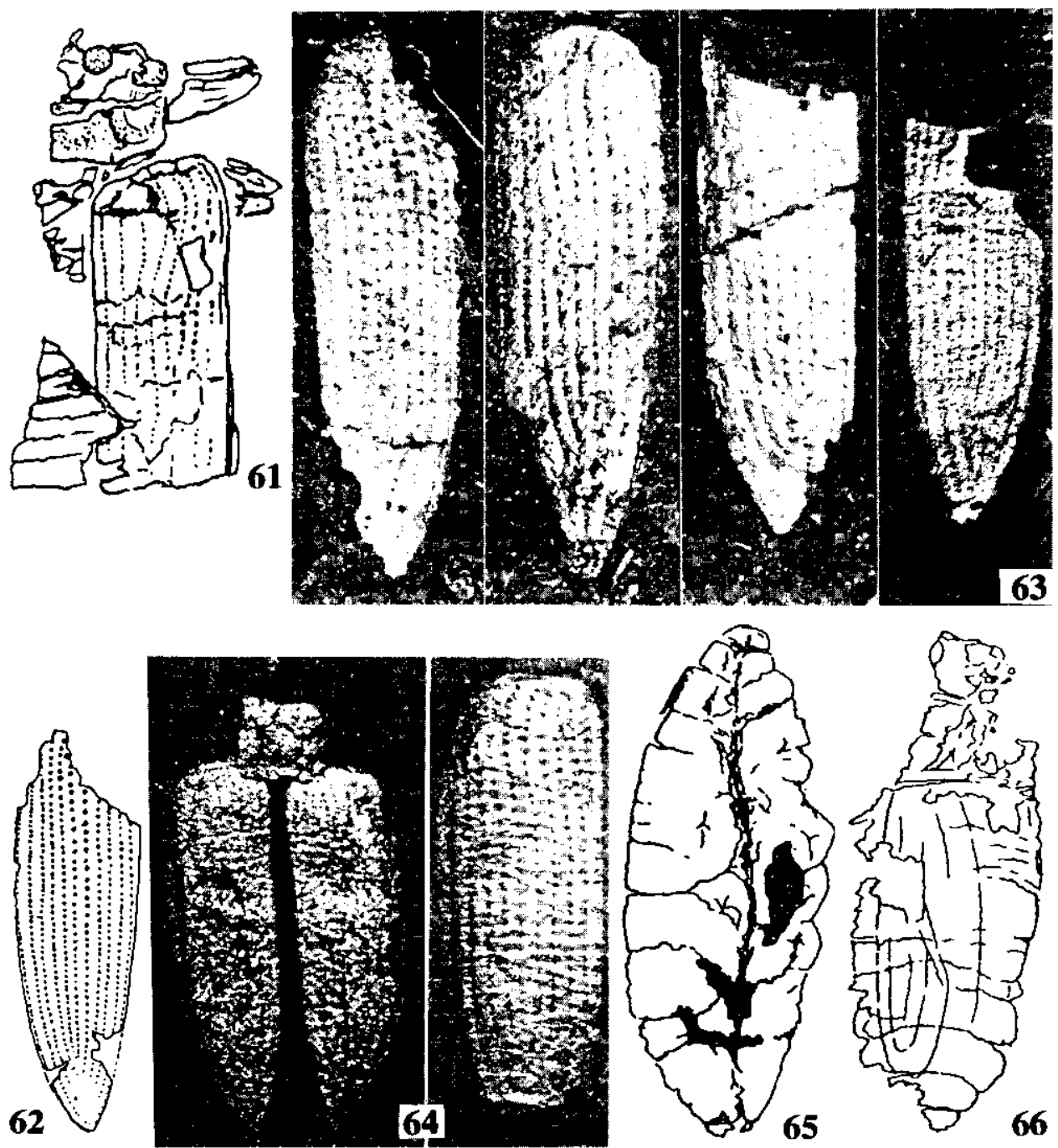

Plate 7. Figs. 61-62. Donacia vicina Haupt, 1956; Fig. 63. D. voigti Goecke, 1943; Fig. 64. D. weigelti Goecke, 1943; Fig. 65-66. Donacia sp., larvae and pupae, respectively. 

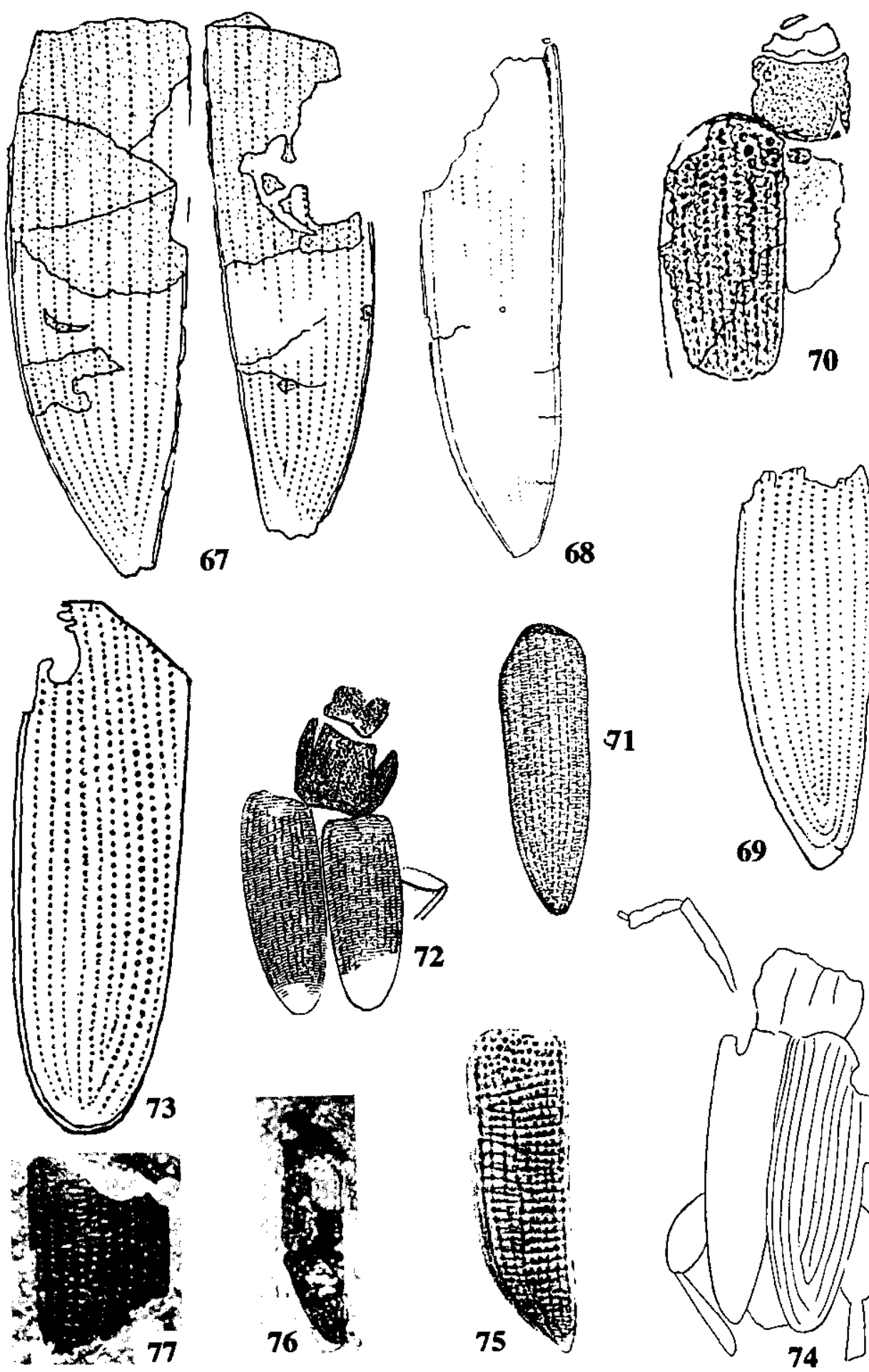

71
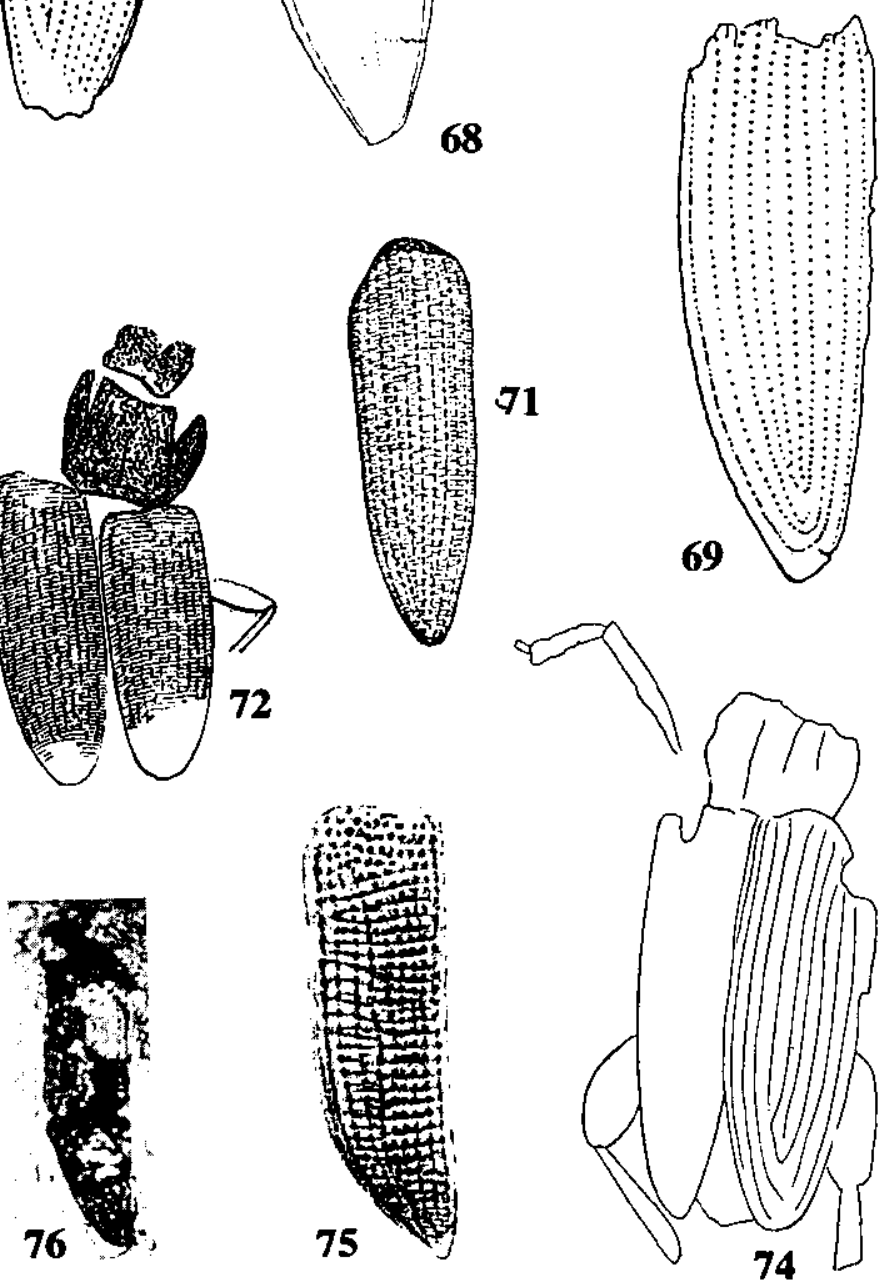

Plate 8. Figs. 67-68. Eodonacia goeckei (Haupt, 1956) (Eodonacia=D. (Donacia), Askevold, 1991); Fig. 69. E. paludosa Haupt, 1956; Fig. 70. Hemidonacia insolita Haupt, 1956 (similar to D. (Cyphogaster) provostii Fairmaire, 1885; Hemidonacia =D. (Cyphogaster), Askevold, 1991); Fig. 71. Plateumaris affinis Kirby, 1837; Fig. 72. P. discolor (Panzer, 1795); Fig. 73. P. fallax Haupt, 1956 (Not a donaciine, Askevold, 1991; is it a chrysomelid?); Fig. 74. P. primaeva (Wickham, 1912) (Fig. 74); Fig. 75. P. sericea (Linné, 1758); Fig. 76. Plateumaris sp.; Fig. 77. Plateumaris sp. or 


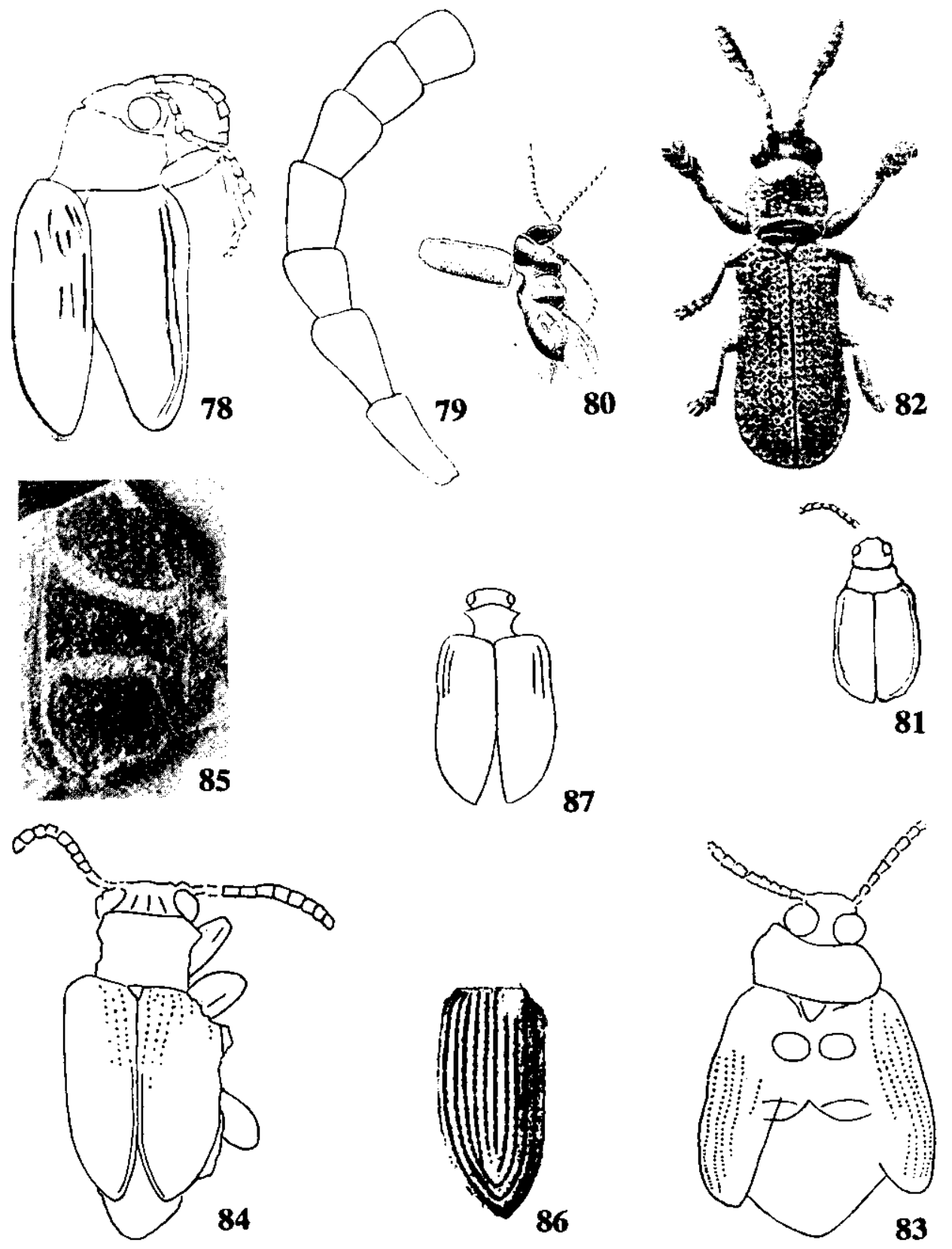

Plate 9. Figs. 78-79. Crioceridea dubia Wickham, 1912. Body, dorsal and antenna, respectively; Fig. 80. Crioceris margarum Oustalet, 1874; Fig. 81. C. vetusta (Heer); Fig. 82. Electrolema baltica Schaufuss, 1892 (From Korschefsky, 1939); Fig. 83. Lema fortior Wickham, 1914b; Fig. 84. L lesquereuxi Wickham, 1914a; Fig. 85. L pervetusta Cockerell, 1921; Fig. 86. L pulchella Förster, 1891; Fig. 87. $L$ tumulata Heyden and Heyden, 1865. 

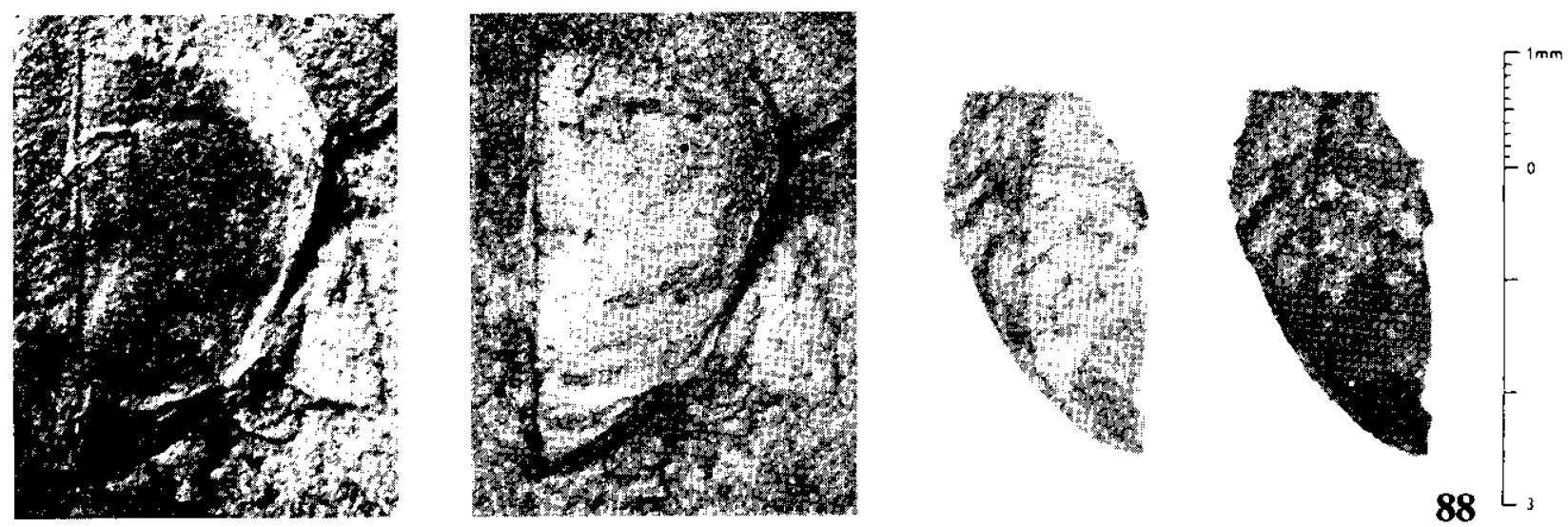

\section{A}

$B$

C

$D$

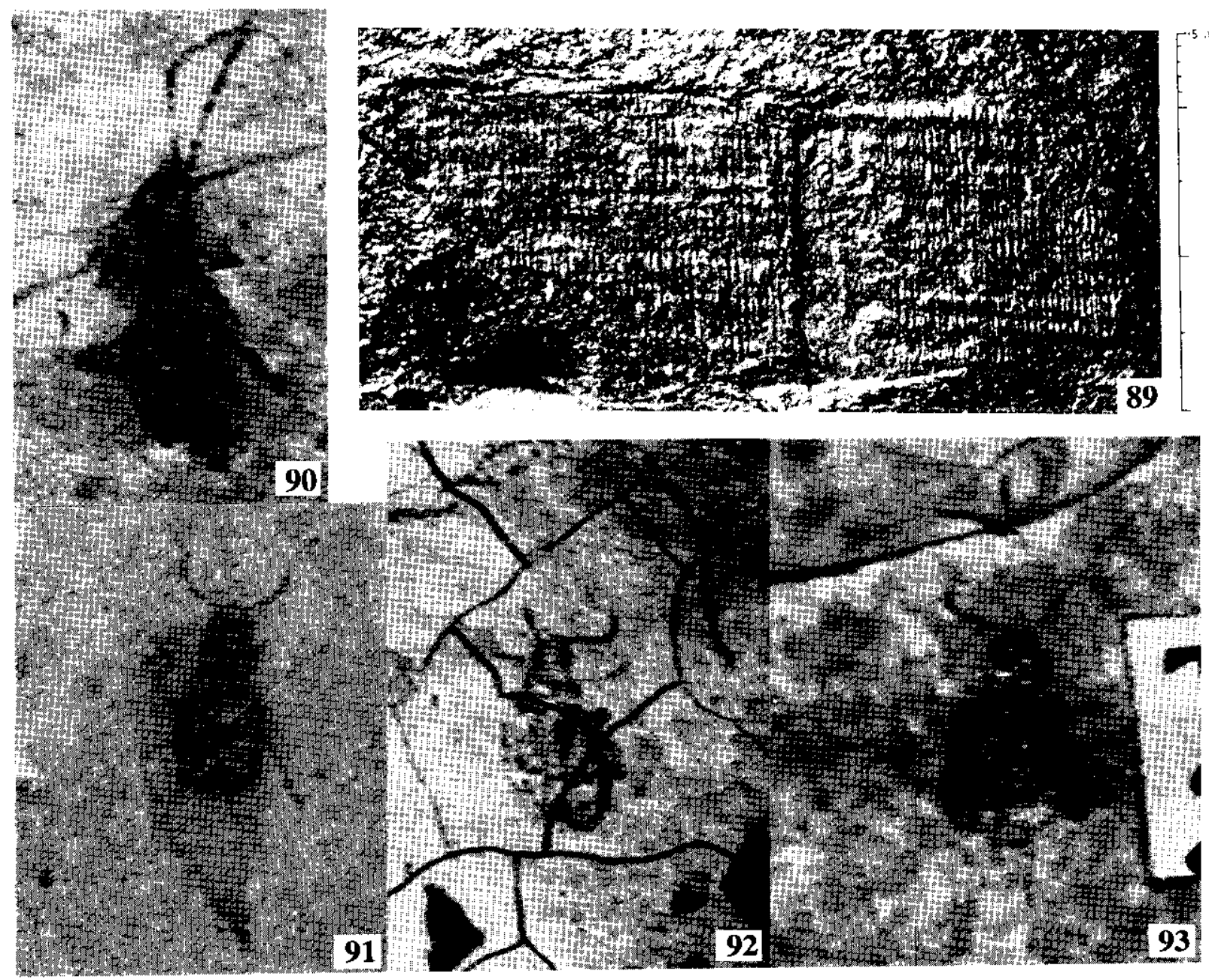

Plate 10. Figs. 88 A-D. Donacia parvula Heer, 1870. (From Birket-Smith, 1977); Fig. 89. D. smittiana Heer, 1870 (From BirketSmith, 1977); Fig. 90. Plateumaris primaeva (Wickham, 1912); Fig. 91. Crioceridea dubia Wickham, 1912; Fig. 92. Lema evanescens Wickham, 1910; Fig. 93. L fortior Wickham, 1914b. 


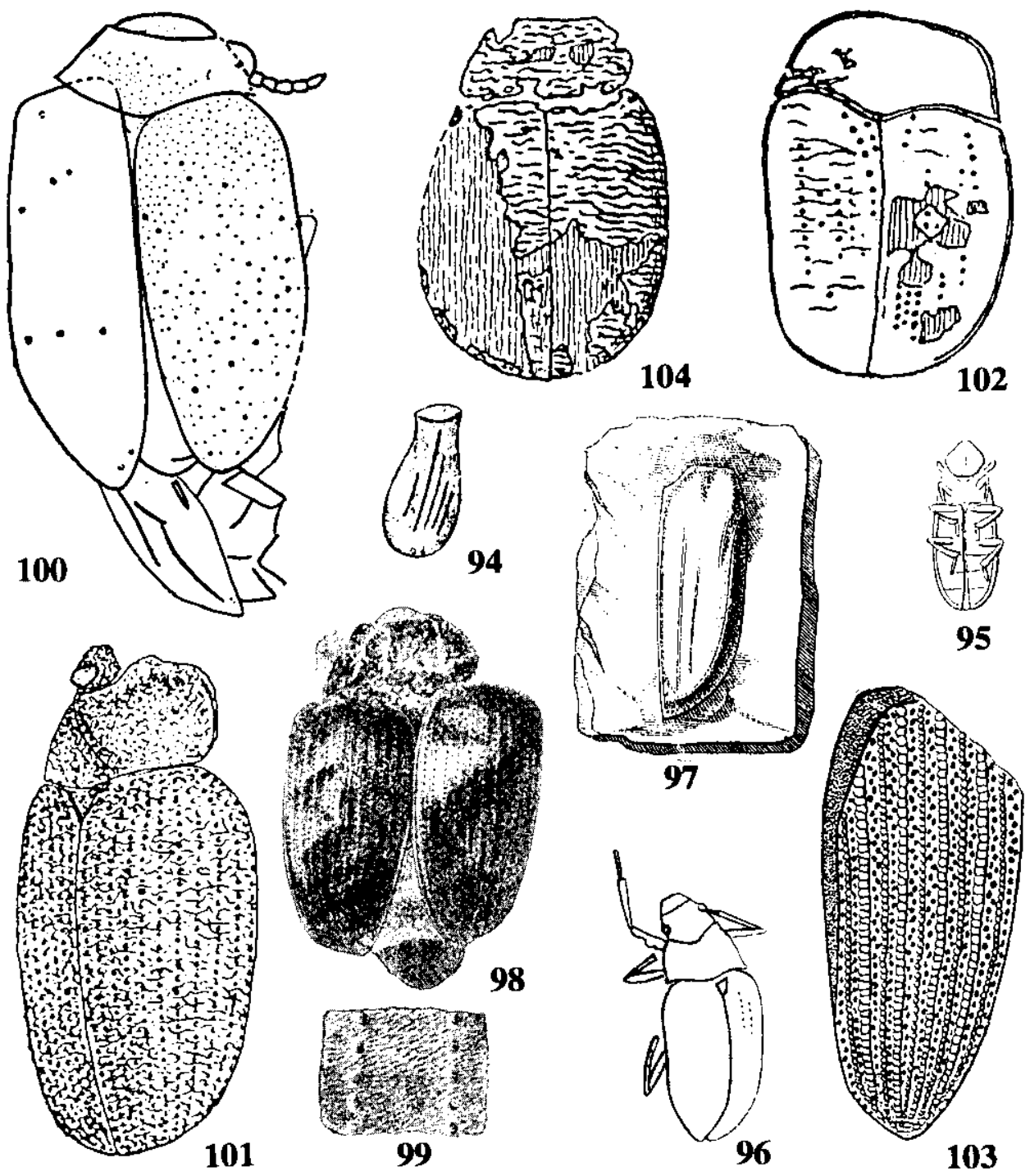

Plate 11. Fig. 94. Larval case of Clytra carbonaria Heyden and Heyden, 1865; Fig. 95. C. pandorae Heer, 1847 (ventral view, not a larval case); Fig. 96. Labidostomis phrrha Heyden and Heyden, 1866; Fig. 97. Clytra greithiana (Heer, 1847) (as Melolontha); Figs. 98-99. Saxinis regularis Scudder, 1898. 98. Body, dorsal. 99. Portion of right elytron; Fig. 100. Smaragdina? incerta Zhang, 1989; Fig. 101. Cryptocephalites auratus Haupt, 1956; Fig. 102. C. elongatus Haupt, 1956; Fig. 103. C. punctatus Scudder; Fig. 104. C. rufiger Haupt, 1956. 


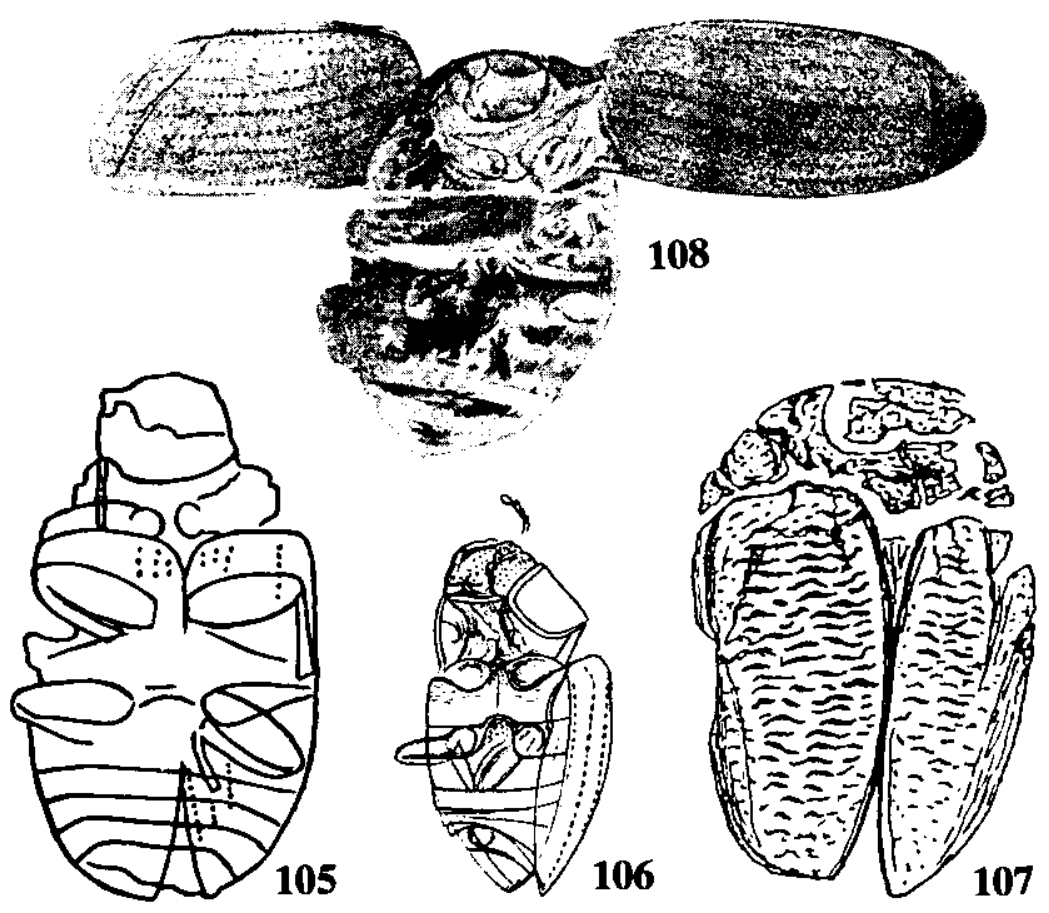

Plate 12. Fig. 105. Cryptocephalus miocenus Wickham, 1913c; Fig. 106. C. relictus Schlechtendal, 1893; Fig. 107. C. rugosus Haupt, 1956; Fig. 108. C. vetustus Scudder, 1878. 

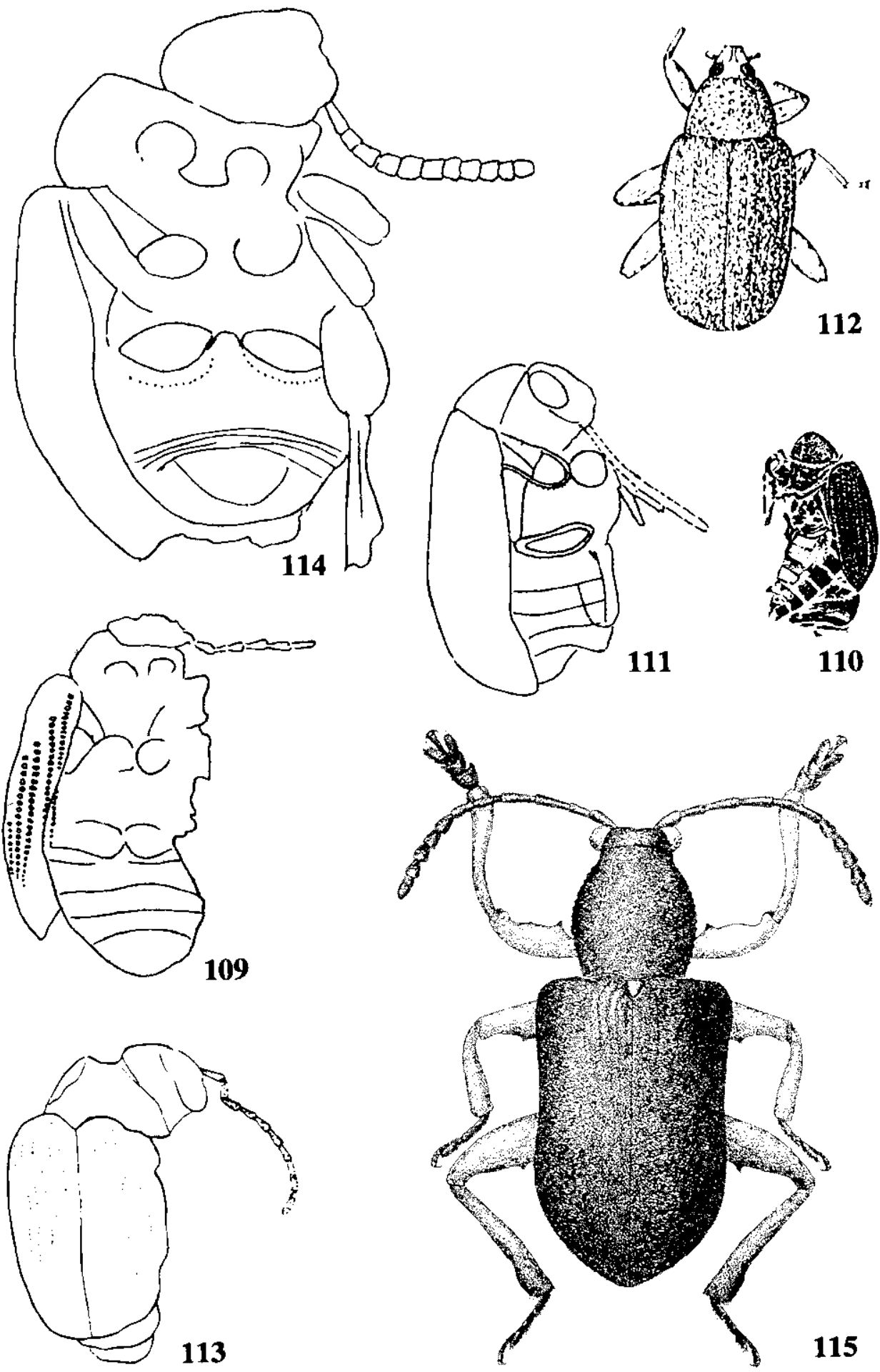

Plate 13. Fig. 109. Colaspis diluvialis Wickham, 1914b. (See also Fig. 116); Fig. 110. C. luti Scudder, 1893. (See also Fig. 117); Fig. 111. C. proserpina Wickham, 1914b. (See also Fig. 118); Fig. 112. Eoeumolpinus azureviridis Haupt, 1950; Fig. 113. Metachroma florissantensis Wickham, 1912; Fig. 114. Prochaetocnema florissantella Wickham, 1914b. (See also Fig. 119); Fig. 115. Profidia nitida Gressitt, 1963. 

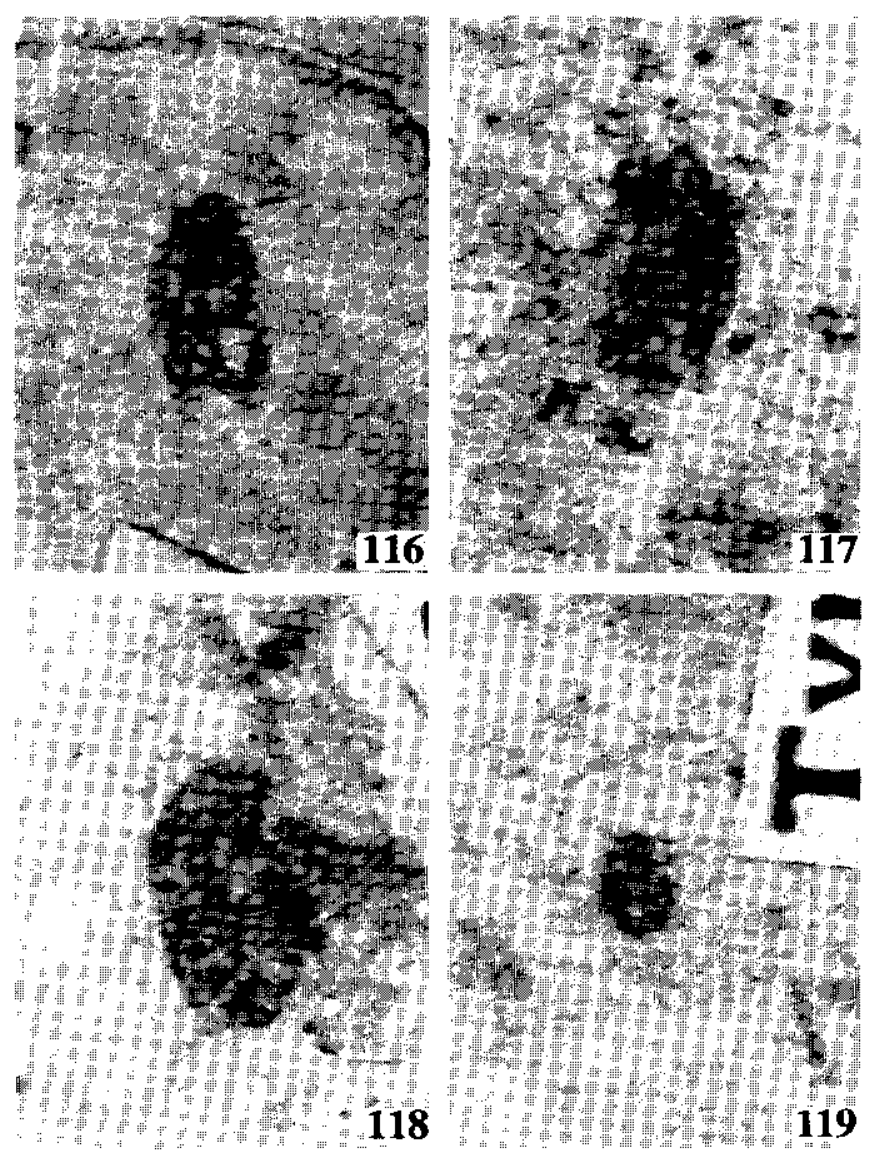

Plate 14. Fig. 116. Colaspis diluvialis Wickham, 1914b. (See also Fig. 109); Fig. 117. C. luti Scudder, 1893. (See also Fig. 110); Fig. 118. C. prosperpina Wickham, 1914b. (See also Fig. 111); Fig. 119. Prochaetocnema florissantella Wickham, 1914. (See also Fig. 114). 

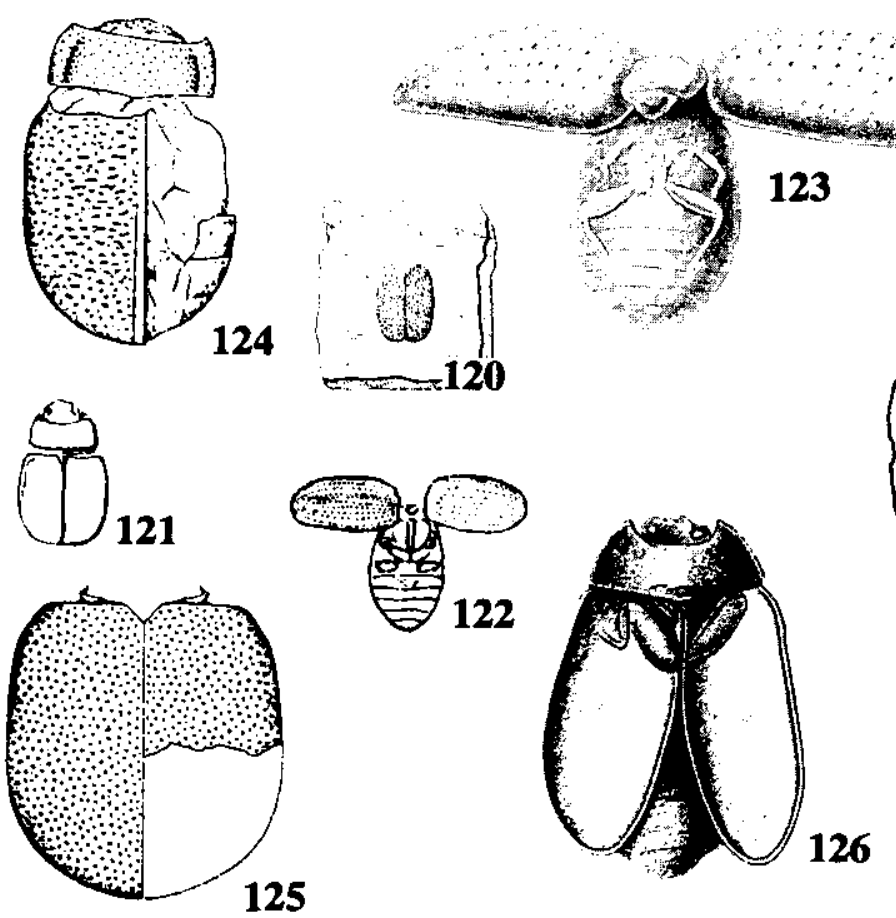

\section{5}
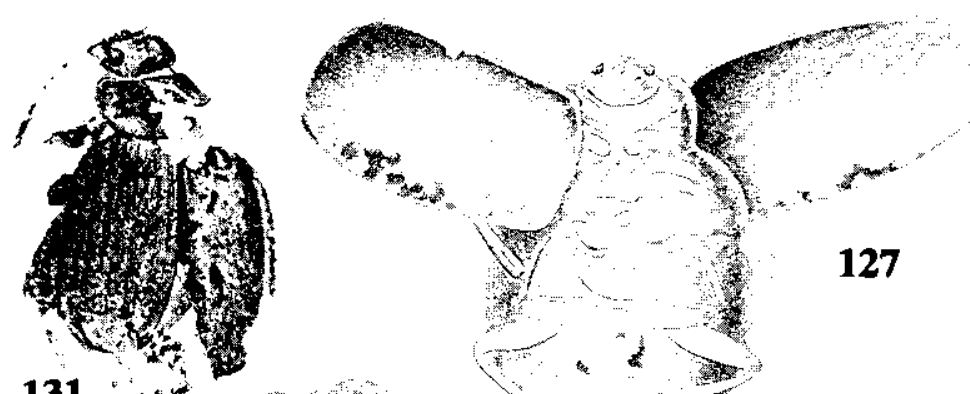

127
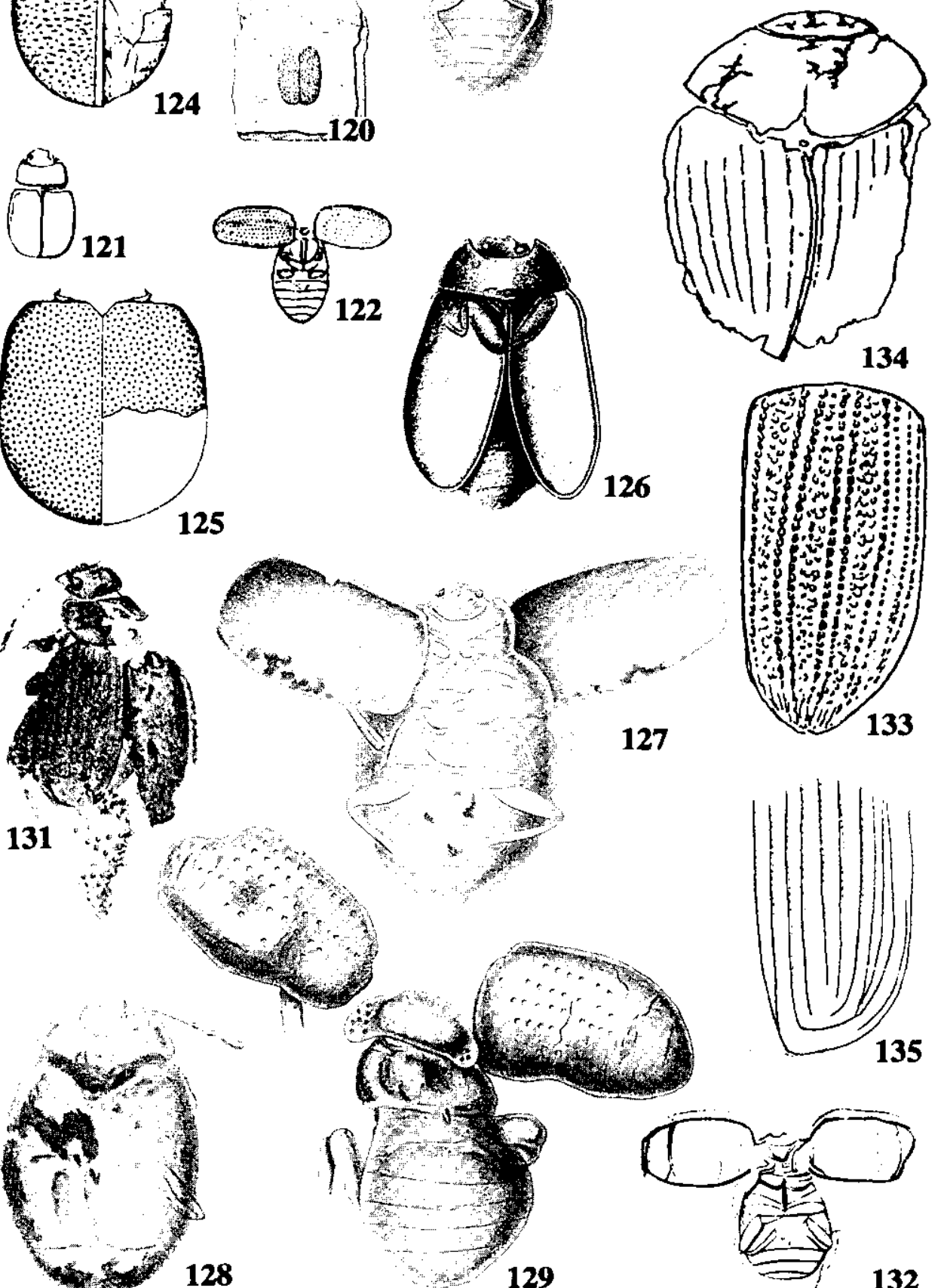

128
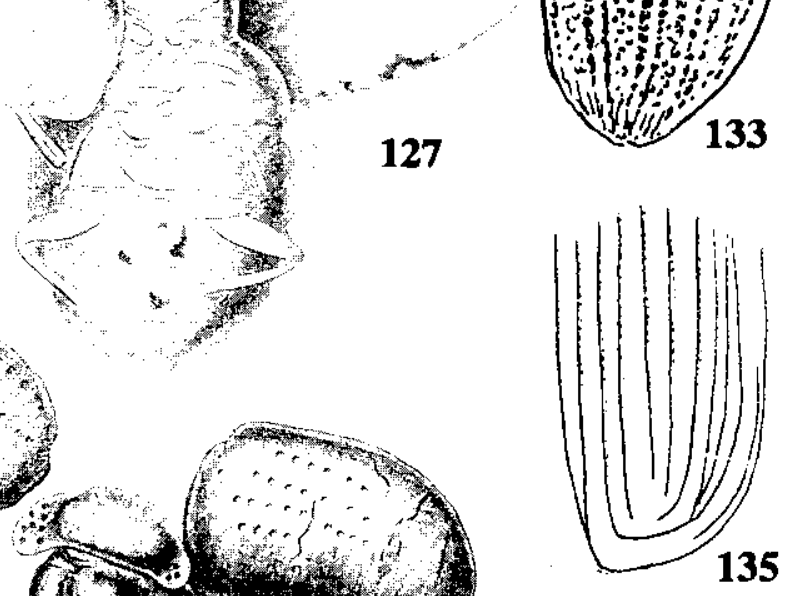

Plate 15. Fig. 120. Chrysochloa sp. (From Théobald, 1937; valid name is Oreina); Fig. 121. Chrysomela calami Heer, 1847. (From Heer, 1872) (Valid name for most of these Chrysomela is Chrysolina); Fig. 122. C. ceresti Théobald, 1937; Fig. 123. C. debilis Oustalet, 1874; Fig. 124. C. hilberi Lomnicki, 1894; Fig. 125. C. lichenis Richter, 1820 (From Lomnicki, 1894; valid name is Chrysolina lichenis); Figs. 126-127. C. lyelliana Heer, 1856a. 126. Dorsal. 127. Ventral. (From Oustalet, 1874); Fig. 128. C. mathrona Oustalet, 1874; Fig. 129. C. matheroni Oustalet, 1874; Fig. 130. C. punctigera Heer, 1847; Fig. 131. C. vesperalis Scudder, 1893. (See also Fig. 136); Fig. 132. Chrysomela sp. (From Curtis, 1829 ); Fig. 133. Chrysomelites allochlamys Cockerell, 1920b; Fig. 134. C. azureus Haupt, 1956; Fig. 135. C. bartonicus Cockerell, 1920 b. 

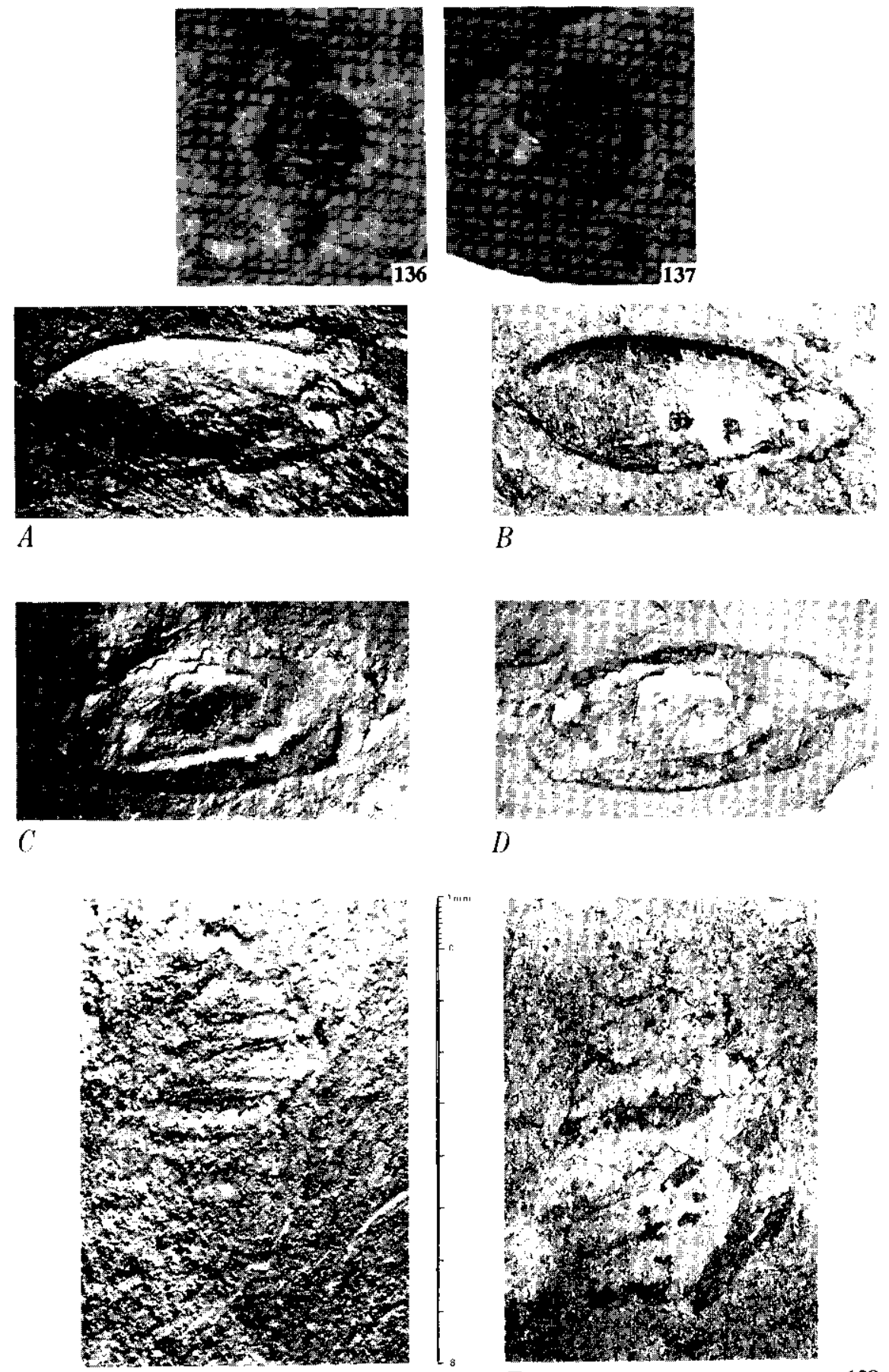

$E$

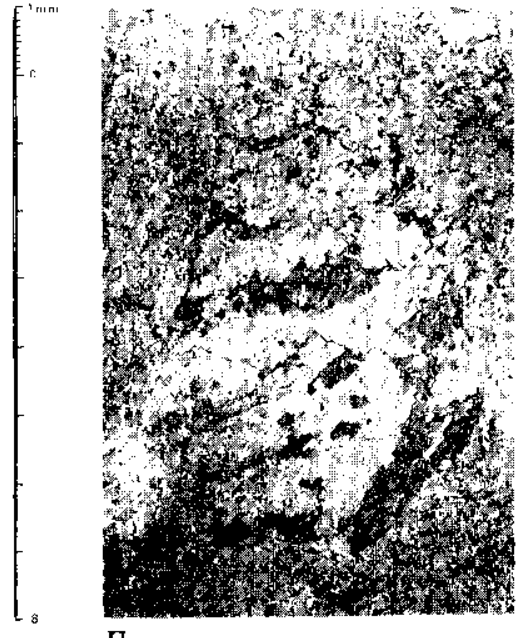

Plate 16. Fig. 136. Chrysomela vesperalis Scudder, 1893. (Valid name for most of these Chrysomela is Chrysolina.) (See also Fig. 131 ); Fig. 137. Chrysomelites alaskanus Heer, 1869; Fig. 138. C. lindhageni Heer, 1870 nomen relictum. (From Birket-Smith, 1977). 


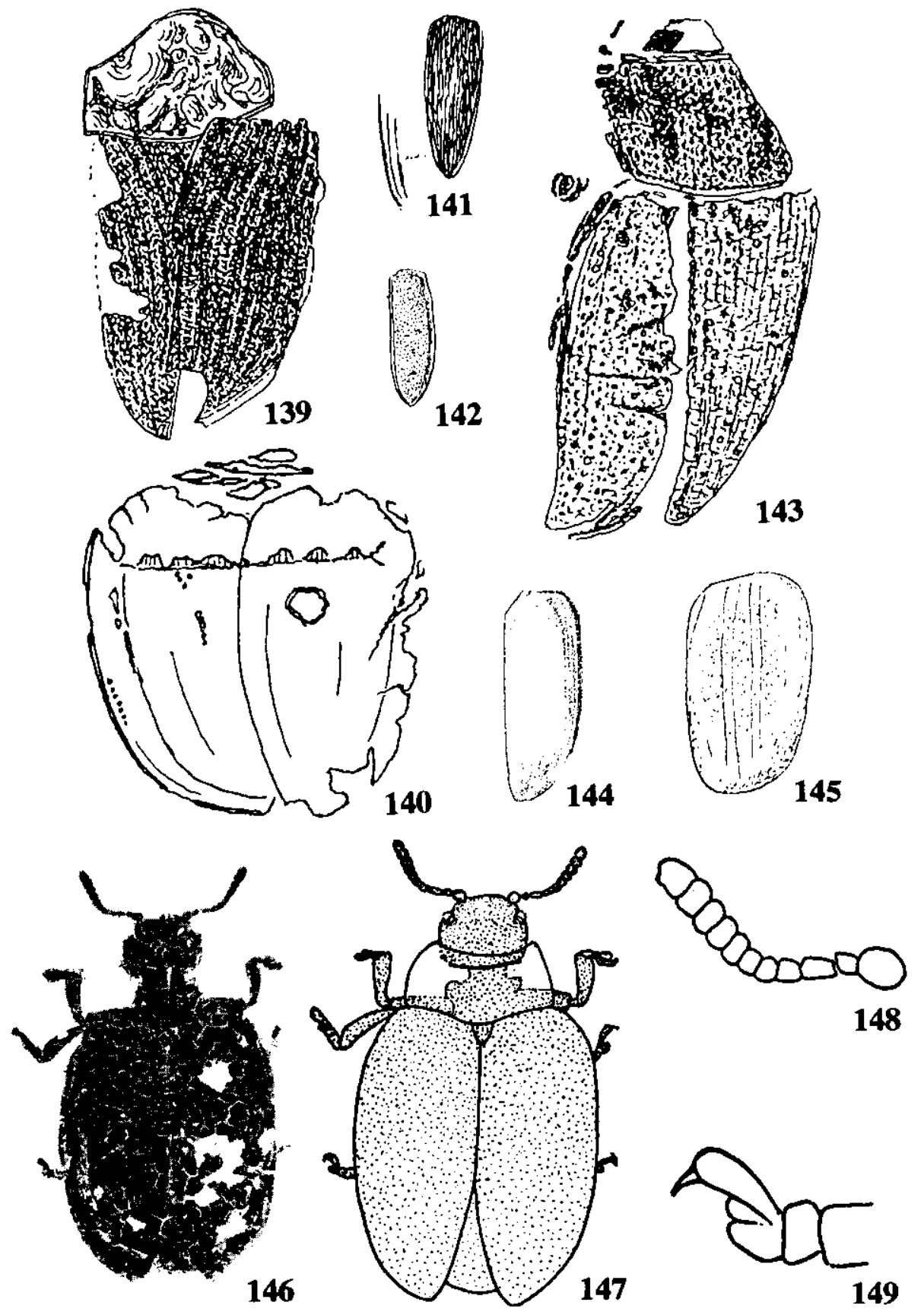

Plate 17. Fig. 139. Chrysomelites bisornatus Haupt, 1956; Fig. 140. C. cupreus Haupt, 1956; Fig. 141. C. danielis Cockerell, 1926; Fig. 142. C. fabricii Heer, 1868; Fig. 143. C. foveolatus Haupt, 1956; Fig. 144. C. lindhageni Heer, 1870 nomen relictum. (See also Fig. 138 A-F); Fig. 145. C. quadrilineatus Cockerell, 1920b; Fig. 146-149. Chrysothoracus tropicus Zhang, 1989. 146, 147. Body, dorsal. 148. Antenna. 149. Tarsi. 


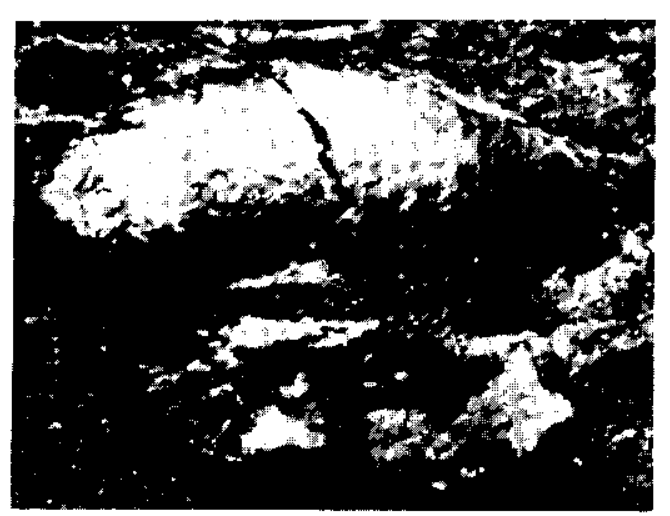

A

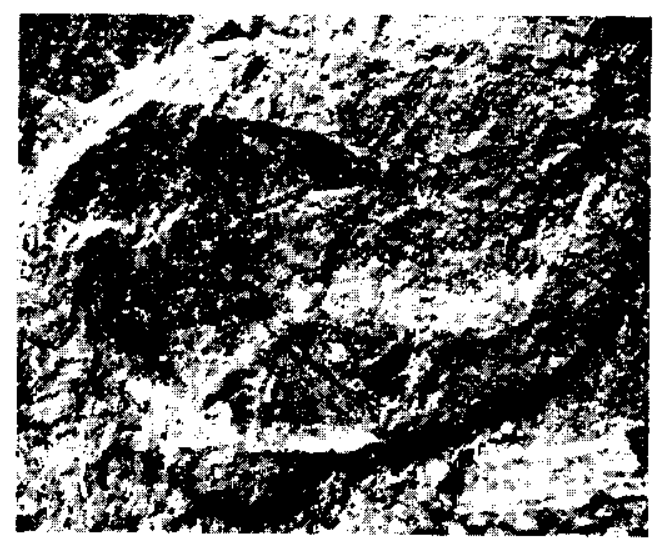

C
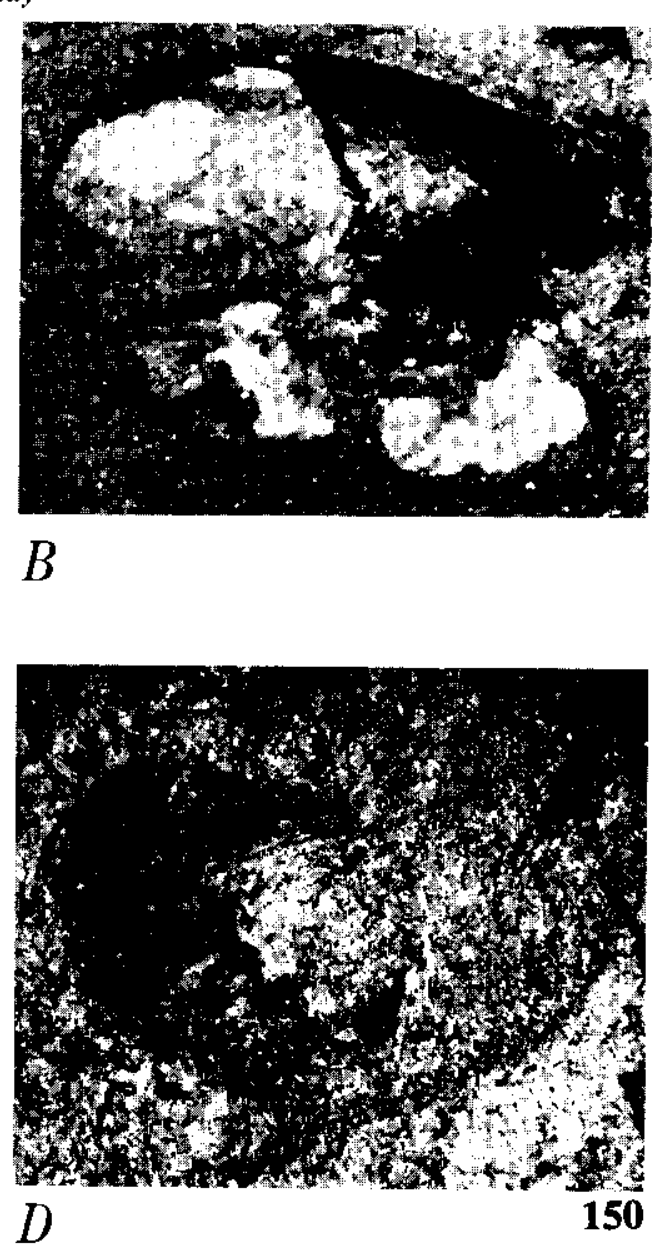

Plate 18. Fig. 150 A-D. Chrysothoracus thulensis Heer, 1870 nomen relictum. (From Birket-Smith, 1977). 

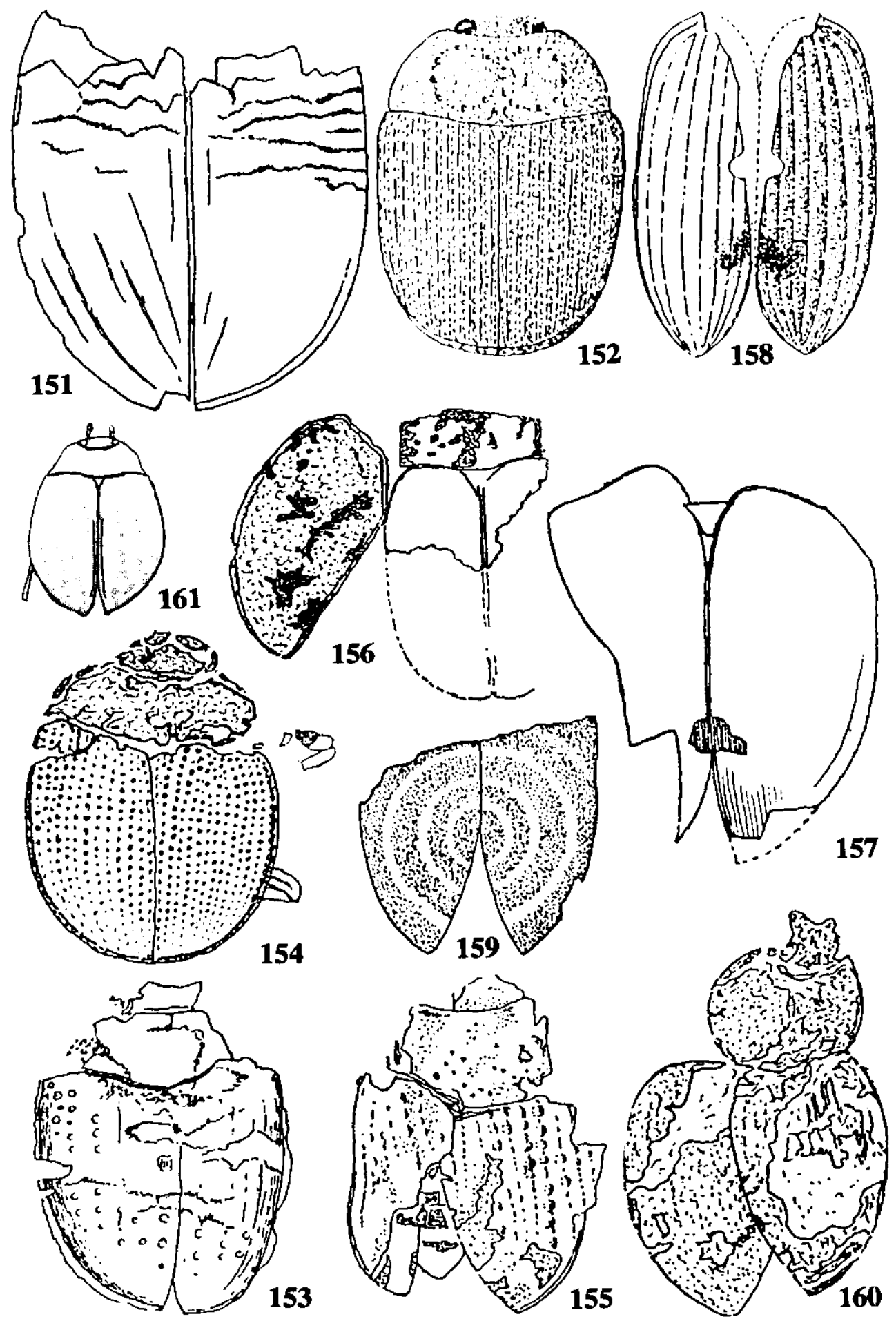

Plate 19. Fig. 151. Eochrysomela indecorata Haupt, 1956; Figs. 152-153. E. ornata Haupt, 1956; Fig. 154. E. punctutor Haupt, 1956; Fig. 155. E. pustulata Haupt, 1956; Fig. 156. Eomelasoma incostata Haupt, 1956; Fig. 157. E. scutellata Haupt, 1956; Fig. 158. Gonocelis notatus Haupt, 1950; Fig. 159. Halocoleus cameratus Haupt, 1950; Fig. 160. Hemisphaerocosites sphaericus Haupt, 1956; Fig. 161. Plagiodera novata (Heyden and Heyden, 1866). 

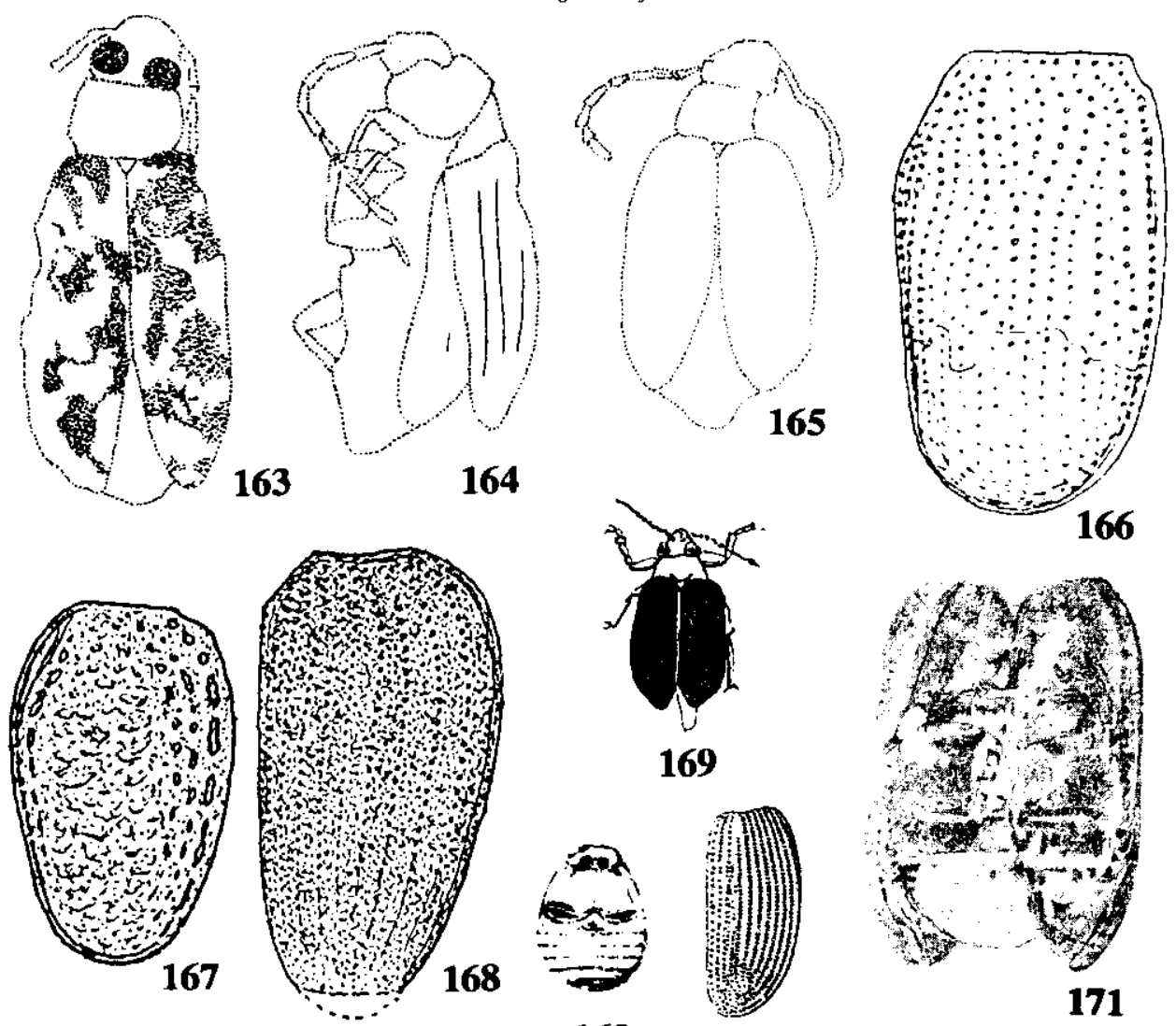

166

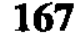

168
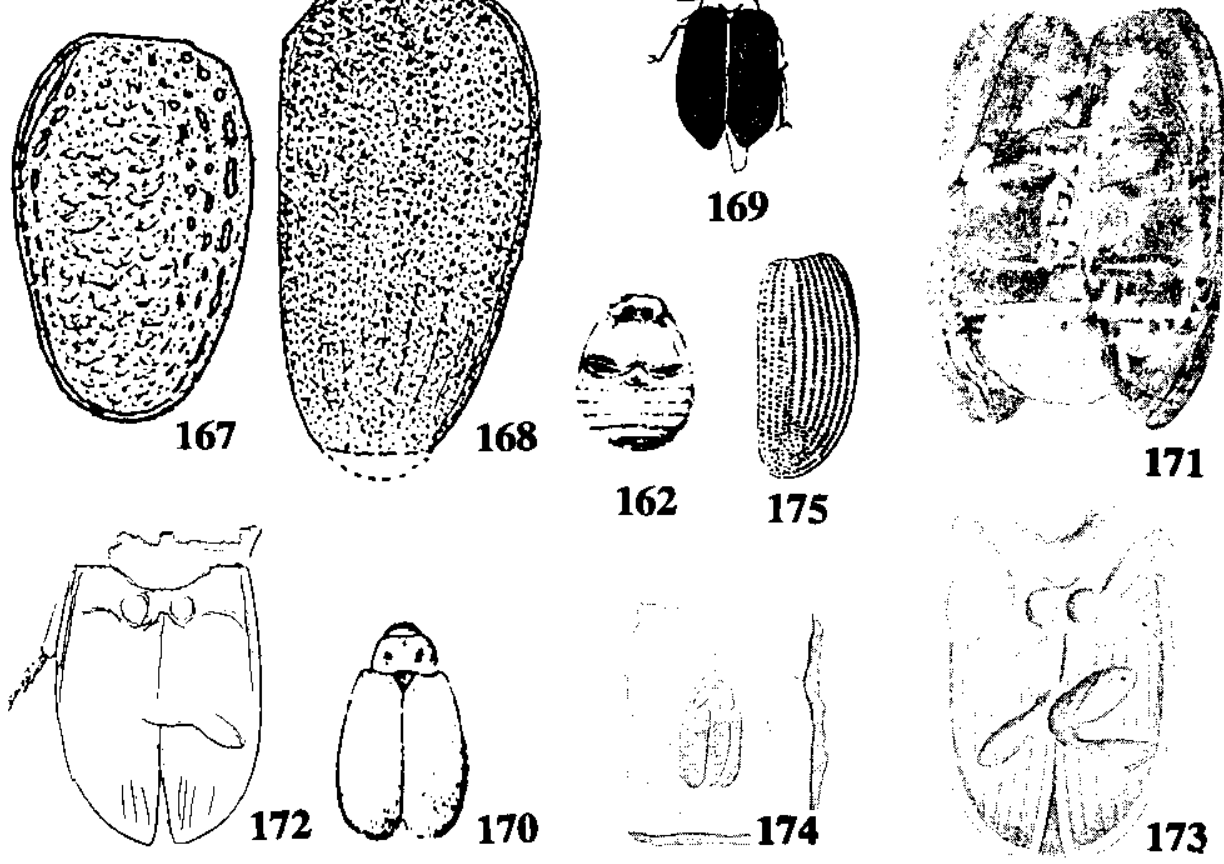

Plate 20. Fig. 162. Agelasa sessilis Förster, 1891; Fig. 163. Diabrotica bowditchiana Wickham, 1914b. (See also Fig. 189); Fig. 164. D. florissantella Wickham, 191b. (See also Fig. 191); Fig. 165. D. uteana Wickham, 1914b. (See also Fig. 192); Fig. 166. Eogaleruca irregularis Haupt, 1956; Fig. 167. E. minor Haupt, 1956; Fig. 168. E. punctipennis Haupt, 1956; Fig. 169. Galeruca buchi Heer, 1872 (as G. buchii); Fig. 170. Galerucella affinis Förster, 1891; Fig. 171. G. picea Scudder, 1879. (From Scudder, 1890a); Fig. 172. Gonioctena clymene Heer, 1847; Fig. 173. G. curtisii Oustalet, 1874; Fig. 174. G. japeti Heer, 1847; Fig. 175. G. primordialis Assmann, 1870. 


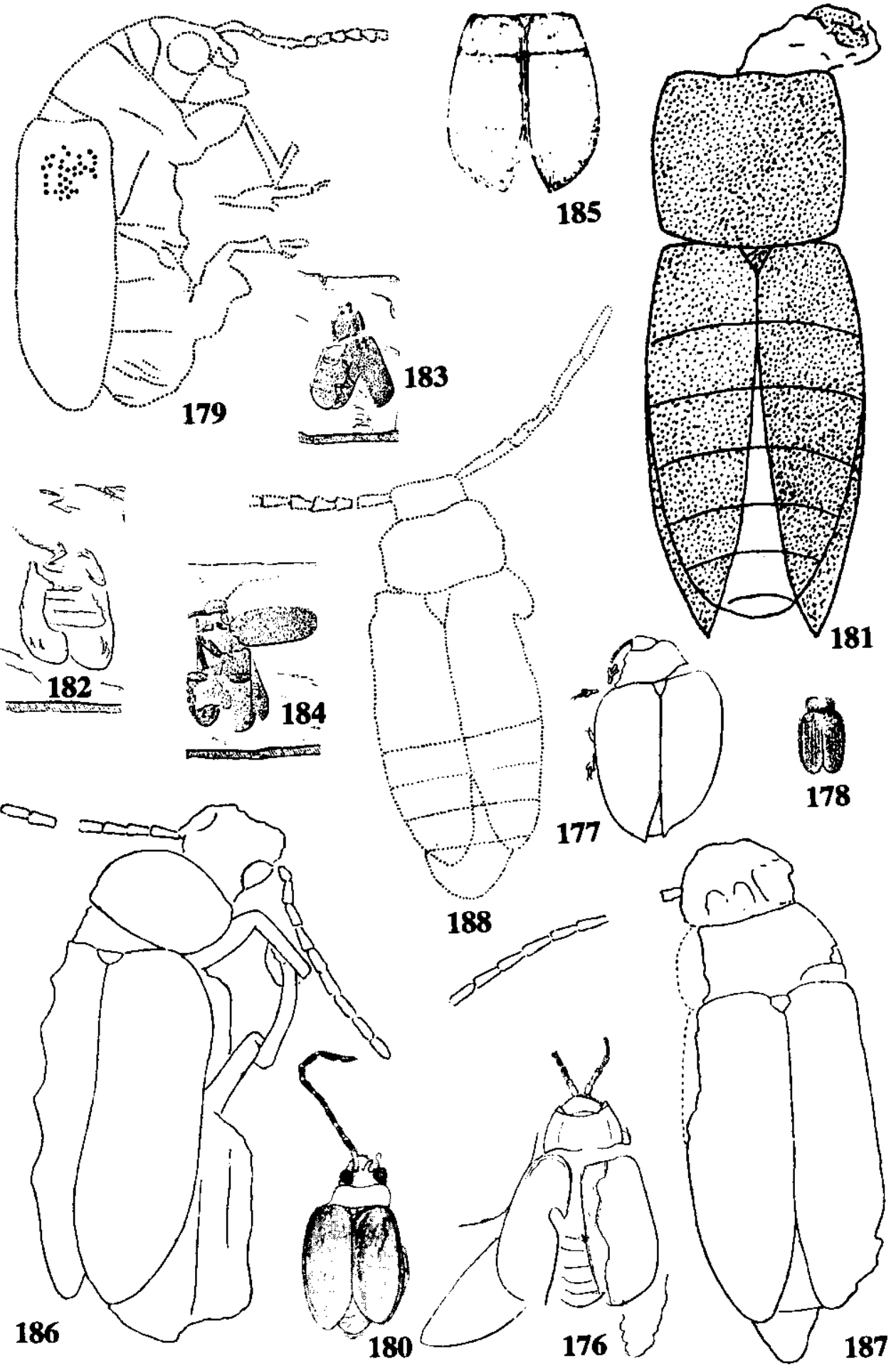

Plate 21. Fig. 176. Lina populeti Heer, 1847 (also as $L$ populi, valid name is Chrysomela populeti); Fig. 177. Lina sociata Heyden and Heyden, 1866, Fig. 178. L. wetteravica Heyden, 1862; Fig. 179. Luperodes submonillis Wickham, 1914a; Fig. 180. Luperus fossilis Schlechtendal, 1893; Fig. 181. Melasoma micropunctata Piton (in Piton and Théobald, 1935; valid name is Chrysomela); Fig. 182. Oreina amphyctionis Heer, 1847 ; Fig. 183. O. hellenis Heer, 1847: Fig. 184. O. protogeniae Heer, 1847; Fig. 185. O. pulchra (Förstcr, 1891); Fig. 186. Trirhabda majuscula Wickham, 1914b (See also Fig. 193); Fig. 187. T. megacephala Wickham, 1914b (See also Fig. 194); Fig. 188. T. sepulla Wickham, 1914b (See also Fig. 195). 

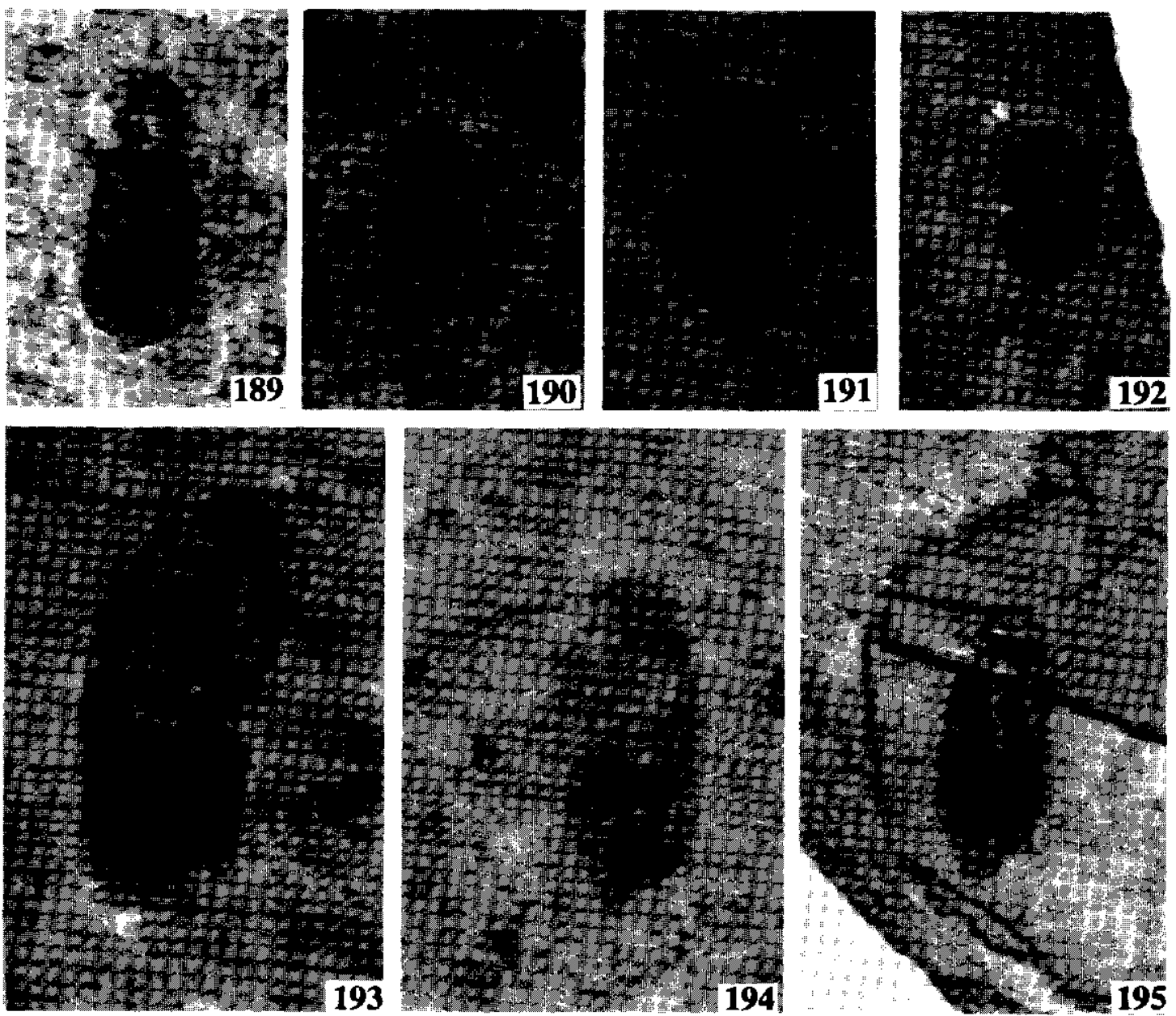

Plate 22. Fig. 189. Diabrotica bowditchiana Wickham, 1914b (See also Fig. 163); Fig. 190. D. exesa Wickham, 1911; Fig. 191. D. florissantella Wickham, 1911b (See also Fig. 164); Fig. 192. D. uteana Wickham, 1914b (See also Fig. 165); Fig. 193. Trirhabda majuscula Wickham, 1914b (See also Fig. 186); Fig. 194. T. megacephala Wickham, 1914b (See also Fig. 187); Fig. 195. T. sepulta Wickham, 1914b (See also Fig. 188). 


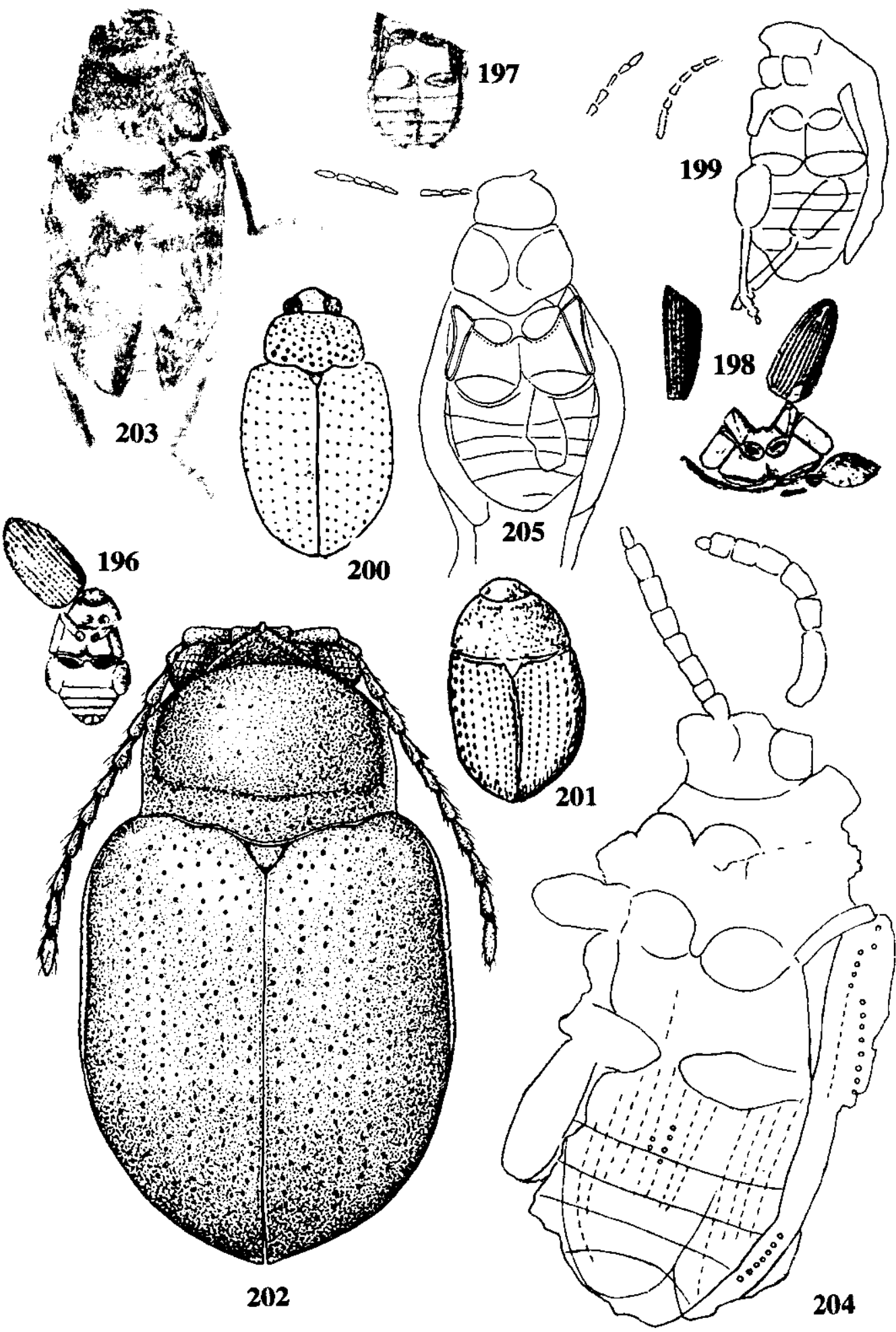

Plate 23. Fig. 196. Altica difficilis Förster, 1891 (as Haltica); Fig. 197. A. dubia Förster, 1891; Fig. 198. A. magna Förster, 1891; Fig. 199. A. renovata Wickham, 1914b (as Haltica renovata). (See also Fig. 206); Fig. 200. Aphthona puncticollis Piton, 1939; Fig. 201. Apteropeda grossa Théobald (in Piton and Théobald, 1935); Figs. 202. Crepidodera antiqua Gressitt, 1971. (valid name is Asiorestia); Fig. 203. Oryctocirtites protogaeum Scudder, 1876. (See also Fig. 207); Fig. 204. Plectrotetrophanes hageni Wickham, 1914b (see also Fig. 208); Fig. 205. Systena florissantensis Wickham, 1913b (See also Figs. 209, 210). 


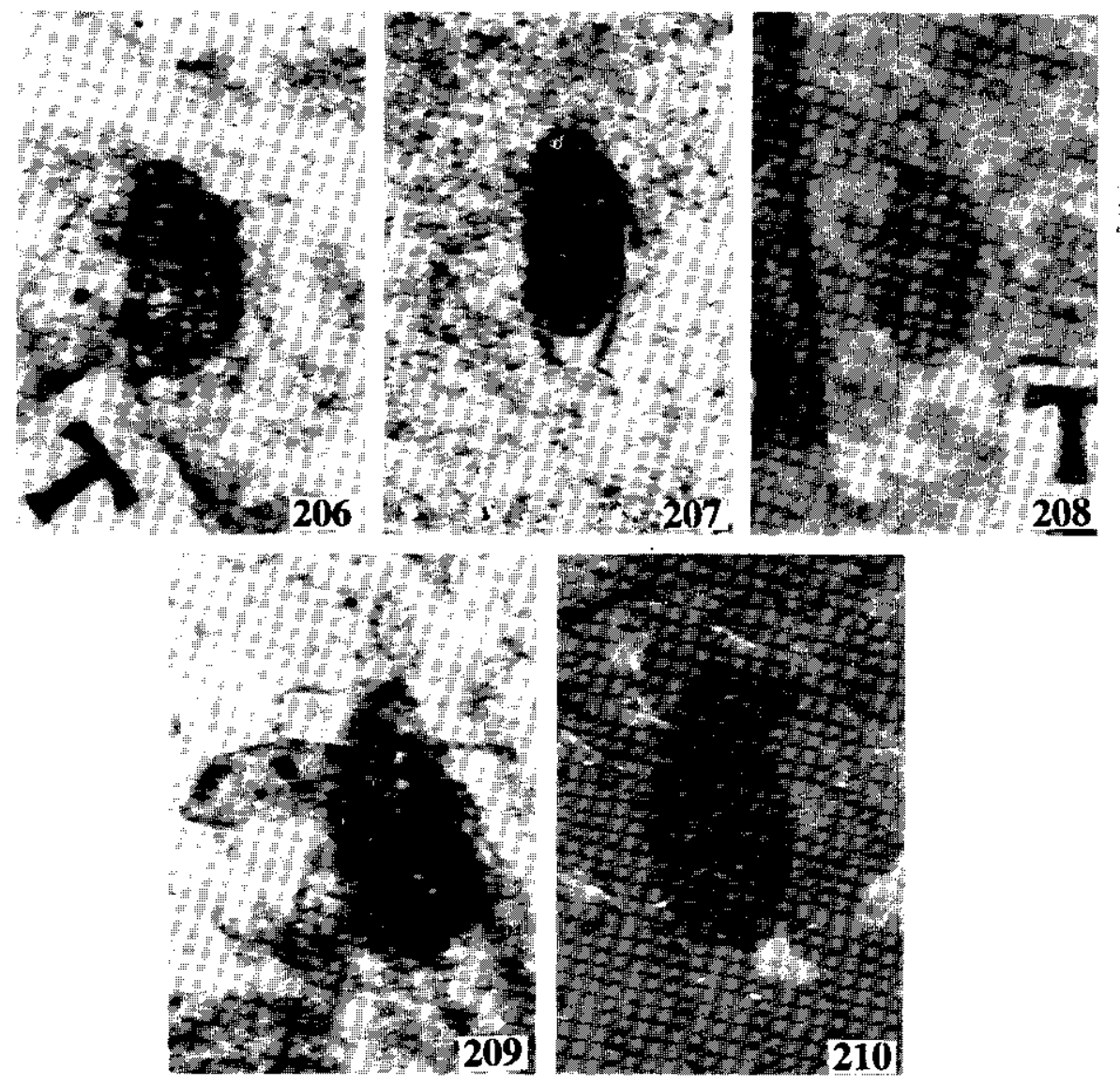

Plate 24. Fig. 206. Altica renovata Wickham, 1914b (as Haltica renovata). (See also Fig. 199); Fig. 207. Oryctocirtites protogaeum Scudder, 1876. (See also Fig. 203); Fig. 208. Plectrotetrophanes hageni Wickham, 1914b (See also Fig. 204); Figs. 209, 210. Systena florissantensis Wickham, 1913b. 210. Dorsal, 211. Ventral. 


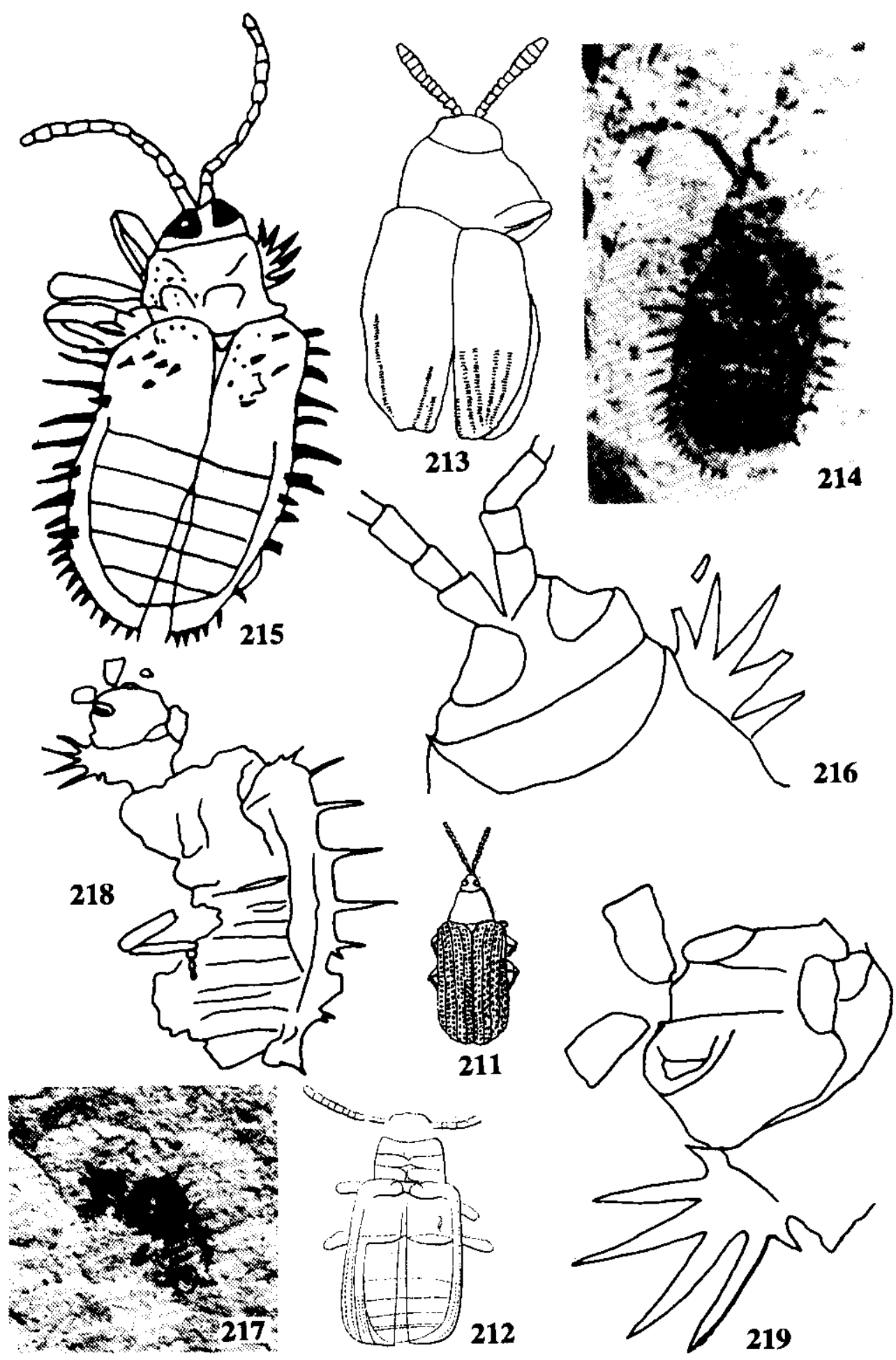

Plate 25. Fig. 211-212. 'Anoplitis' bremii Heer, 1847 (Fig. 211 from Heer, 1872); Figs. 213. Chalepus americanus (Wickham, 1914b) (as Odontota americana Wickham, 1914b) See also Fig. 238); Figs. 214-216. Dicladispa bes-konakensis Nel, 1988; Figs. 217-219. D. muratensis Nel, 

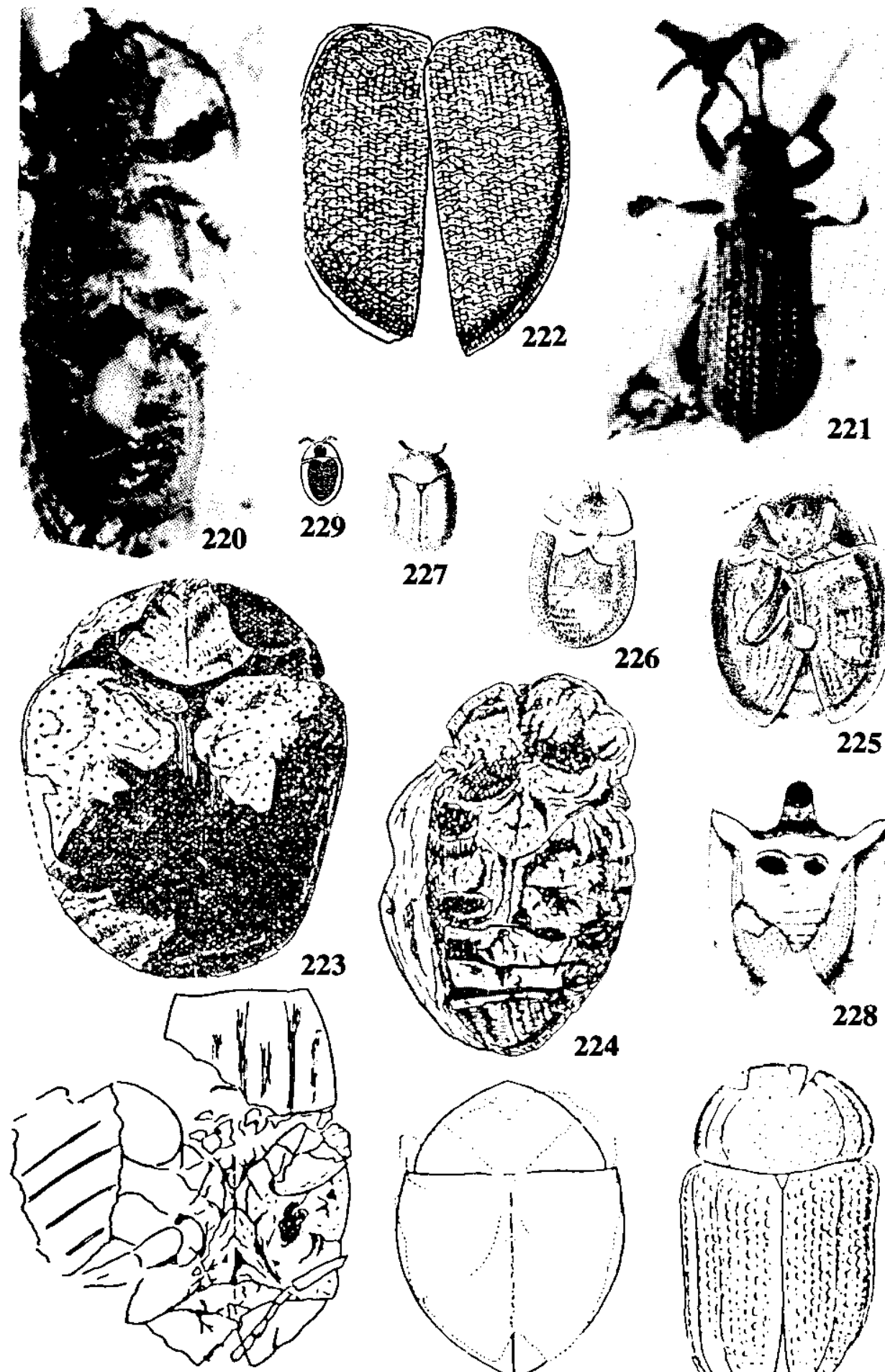

227

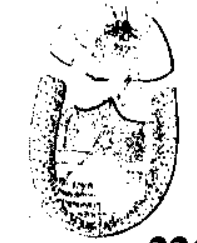

226
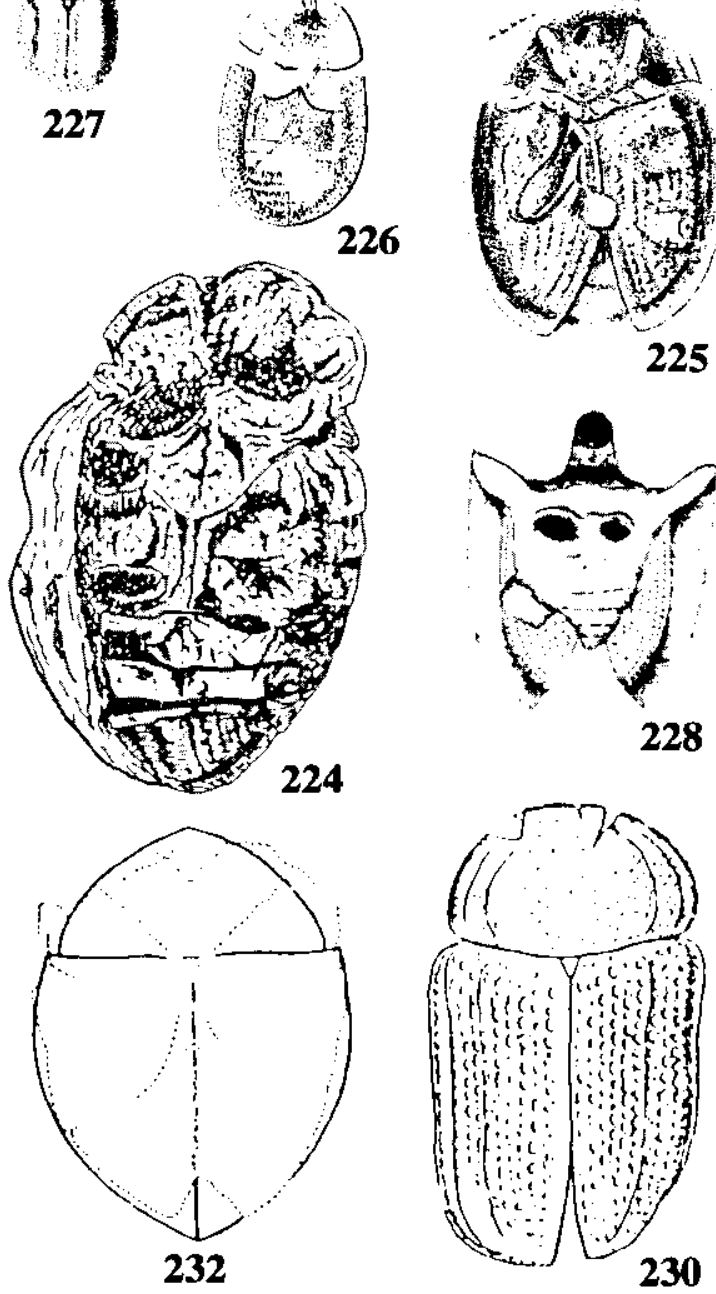

Plate 26. Fig. 220. Oposispa scheelie Uhmann, 1939; Fig. 221. Sucinagonia javetana Uhmann, 1939. (From Larsson, 1978); Fig. 222. Acassidites separandus Haupt, 1950; Figs. 223-224. Callistaspis punctatus Haupt, 1950; 223. Dorsal. 225, Ventral; Fig. 225. Cassida blancheti Heer, 1856a. (From Oustalet, 1874); Fig. 226. C. hermione Heer, 1847; Fig. 227. C. interemta Heyden, 1862; Fig. 228. C. kramstae Förster, 1891; Fig. 229. C. megapenthos Heer, 1847 (also as C. megapentos; Fig. 230. Eocassida longula Haupt, 1950; Figs. 231-232. Mesomphalia gemmaspis Pongr., 1935; 231. Ventral. 232. Dorsal. (From Haupt, 1950). 

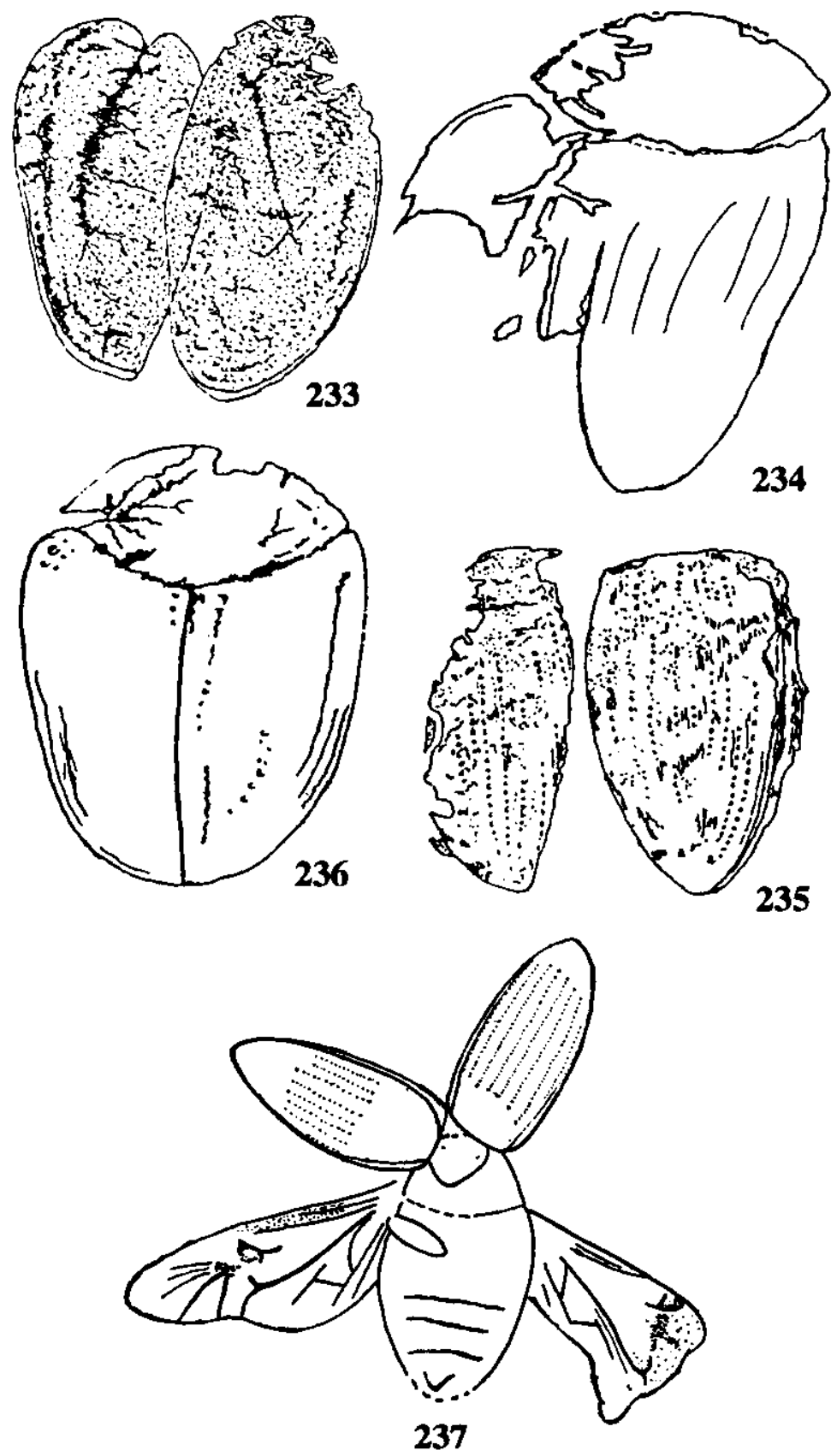

Plate 27. Fig. 233. Paracassida aurichalcea Haupt, 1956; Fig. 234. P. bisangulata Haupt, 1956; Fig. 235. P. detrita Haupt, 1956; Fig. 236. P. punctillata Haupt, 1956; Fig. 237. Unidentified chrysomelid. (From Zhang, 1979). 


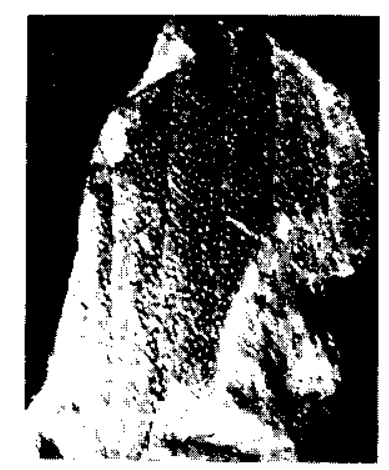

A

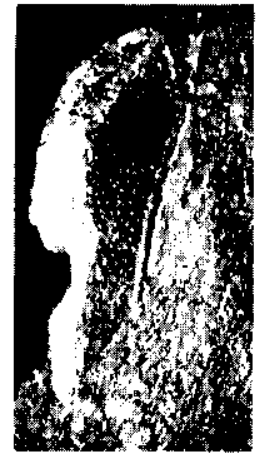

$B$

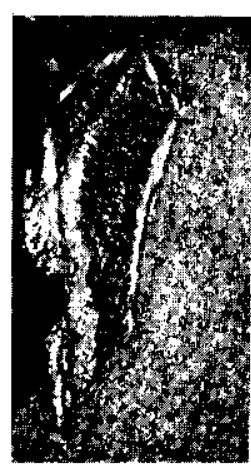

E

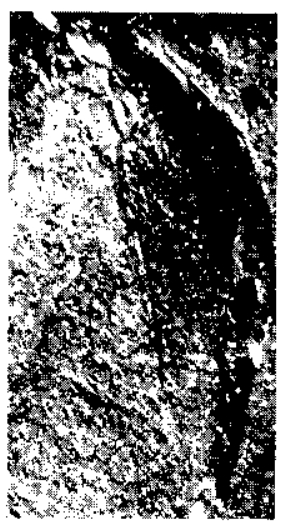

C

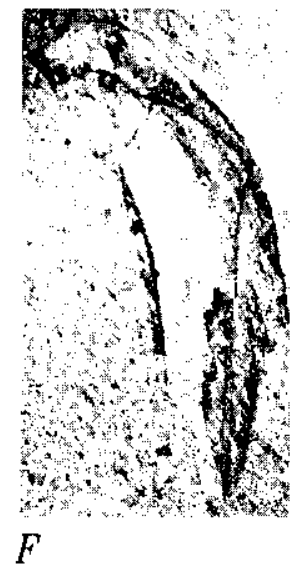

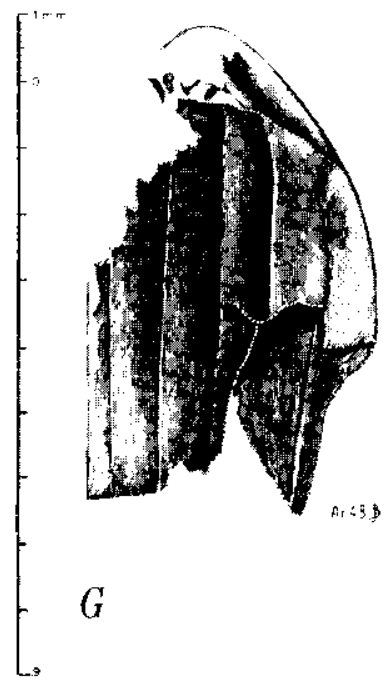
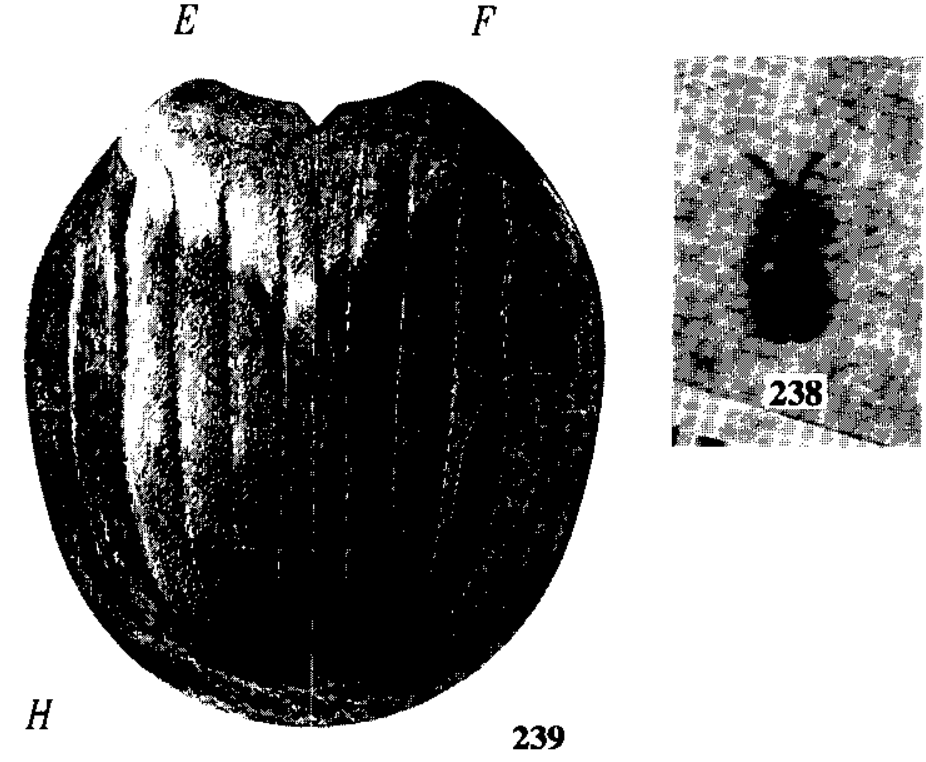

Plate 28. Fig. 238. Chalepus americanus (Wickham, 1914b). (as Odontota americana Wickham, 1914b) See also Fig. 213); Fig. 239A-H. Coelocassida scubriusculum (Heer, 1870). 

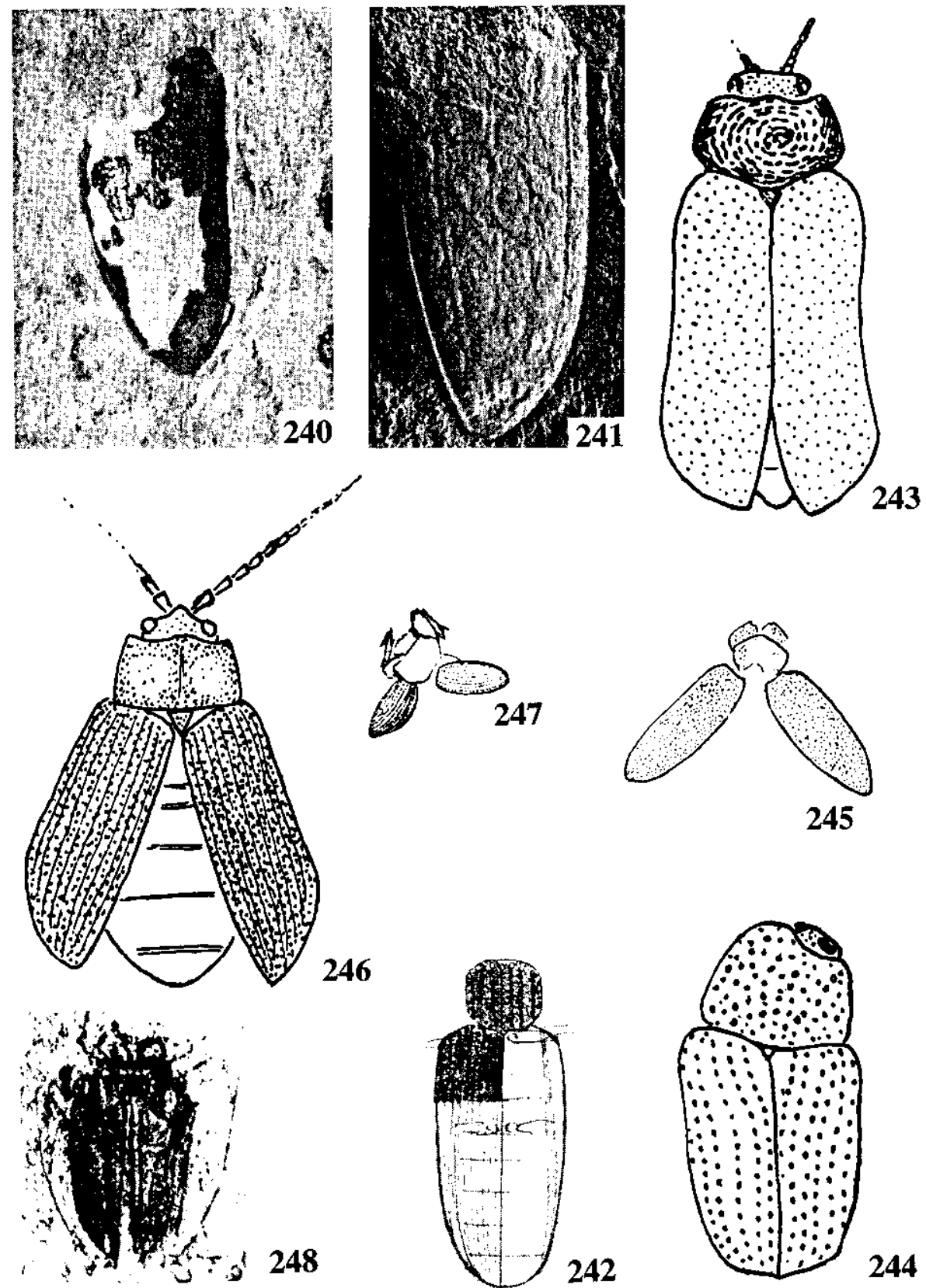

Plate 29. Fig. 240, 241. possible Eumolpinae, resembling Pilacolaspis (from Craw \& Watt, 1987); Fig. 242. Donacia dubia Théobald, 1937; Fig. 243. Clytrina eocenica Piton, 1940; Fig. 244. Crytocephalus minusculus Piton, 1940; Fig. 245. Galerucella emarginata Théobald, 1937; Fig. 246. Altica dryophyllorum Piton, 1940 (as Haltica); Fig. 247. Psylliodes defiguratus Théobald, 1937; Fig. 248. Oligocassida melaena Théobald, 1937. 


\section{References}

Ander, K. E. V., 1942. Die Insektenfauna des Baltischen Bernsteins Nebst damit verknüpften zoogeographischen Problemen. Lunds Universitets Årsskrift, N. F. Avd. 2. Bd. 38. Nr. 4. Kungl. Fysiografiska Sällskapets Handlingar. N. F Bd. 53. Nr. 4. 82 pp.

Andersson, G., 1889. Studier öfver Torfmossar i södra Skane. Bih. till Kongl. Svenska vetensk.-akad. handl. 15:1-43.

Anonymous, 1988. Florissant Fossil Beds National Monument. Bull. South. Calif. Palcontol. Soc. 20:228-234.

Arnoldi, L. V., Zherikhin, V. V., Nikritin, L. M. \& Ponomarenko, A. G., 1977. Mesozoic Coleoptera. Trudy paleont. Inst. 161A:1204.

Ashworth, A. C., 1972a, Deformed head of a fossil Plateumaris sericea L. (Col., Chrysomelidae). Entomol. Monthly Mag. 108:65.

Ashworth, A. C., 1972b. A late-glacial insect fauna from Red Moss. Lancashire, England. Entomol. Scand. 3:211-224.

Ashworth, A. C., 1980. Environmental implications of a beetle assemblage from the Gervais Formation (Early Wisconsinan?), Minnesota. Quatern. Res. 13:20-212.

Ashworth, A. C. \& Brophy, J. A., 1972. Late Quaternary fossil beetle assemblage from Missouri Coteau, North Dakota. Bull. Geol. Soc. Amer. 83:2981-2988.

Ashworth, A. C., Clayton, L. \& Bickley, W. B., 1972. The Mosbeck site: a paleoenvironmental interpretation of the Late Quaternary history of Lake Agassiz based on fossil insect and mollusk remains. Quatern. Res. 2:176-188.

Ashworth, A. C. \& Hoganson, J. W., 1983. Testing the Late Quaternary climatic record of southern Chile with evidence from fossil Coleoptera. ASAQUA Intern. Symp. Swaziland. (29 Aug.-2 Sept. 1983). pp. 85-102.

Ashworth, A. C. \& Hoganson, J. W., 1987. Coleoptera bioassociations along an elevational gradient in the lake region of southern Chile, and comments on the postglacial development of the fauna. Ann. Entomol. Soc. Amer. 80:865-895.

Ashworth, A. C., Markgraf, V. \& Villagran, C., 1991. Late Quaternary climatic history of the Chilean Channels based on fossil pollen and beetle analyses, with an analysis of the modern vegetation and pollen rain. J. Quatern. Sci. 6:279-291.

Ashworth, A. C. \& Schwert, D. P., 1992. The Johns Lake site: a late Quaternary fossil beetle (Coleoptera) assemblage from the Missouri Coteau of North Dakota. Proc. F. D. Holland, Jr. Geol. Symp. J. M. Erickson and J. W. Hoganson (eds.) North Dakota Survey Misc. Ser. 76:257-265.

Ashworth, A. C., Schwert, D. P., Watts, W. A. \& Wright Jr., H. E., 1981. Plant and insect fossils at Norwood in south-central Minnesota: a record of tate-glacial succession. Quatern. Res. $16: 66-79$

Askevold, I. S., 1990a. Classification of Tertiary fossil Donaciinae of North America and their implications about evolution of the Donacinae (Coleoptera: Chrysomelidae). J. Canad. Zool. 68:2135-2145.

Askevold, I. S., 1990b. Reconstructed phylogeny and reclassification of the genera of Donaciinae (Coleoptera: Chrysomelidac). Quest. Entomol. 26:601-664.

Askevold, I. S., 1991. Classification, reconstructed phylogeny, and geographic history of the New World members of Plateumaris Thompson, 1859 (Coleoptera: Chrysomelidae, Donaciinae). Mem. Entomol. Soc. Canada. 157. 175 pp.

Assmann, A., 1870. Paleontologie. Beiträge zur Insekten-Fauna der Vorwelt. Einleitung. I. Beitrag. Die fossilen Insekten des tertiären (miocenen) Thonlagers von Schossnitz bei Kanth in Schlcisien. II. Beitrag. Fossile Insekten aus der tertiären (oligocenen) Braunkohle von Naumburg am Bober. Mit einer Tafel Abbildungen. Breslau. $62 \mathrm{pp}$.

Bachofen-Echt, A. von, 1949. Bernstein und seine Einschlüsse. Wien. 244 pp.

*Baker, [A. C.], 1920. The life of Glacial Lake Chicago. Bull. Illinois Univ. $95 \mathrm{pp}$.

Baker, R. G., Schwert, D. P., Bettis III, E. A., Kemmis, T. J., Norton, D. G. \& Semken, H. A., 1991. Mid-Wisconsian stratigraphy and paleoenvironments at the St. Charles site in southcentral Iowa. Bull. Geol. Soc. Amer. 103:210-220.

Bell, A., 1888. Post-glacial insects. The Entomologist 21:1-2.

Bell, A., 1922. On the Pleistocene and later Tertiary British insects. Ann. Rep. Yorkshire Phil. Soc. 1921:42-51.

Bell, A. \& Bell, R., 1873 ["1870-1871"]. On the English Crags and the stratigraphical divisions indicated by their invertebrate fauna. Proc. Geol. Assoc. (London) 2:185-218.

Benassi, P., 1896. Piante ed insetti di Re in Val Vigezzo. Riv. Ital. Pal. 2:315-320.

*Berendt, G. C., 1830. Die Insekten in Bernstein. Ein Beitrag zur Thiergeschichte der Vorwelt. $1^{\text {es }}$ heft. Danzig.

Berendt, G. C., 1845. Die im Bernstein befindlichen Organischen Reste der Vorwelt gesammelt in Verbindung mit Mehreren bearbeitet und herausgegeben. 1. Band. I. Abd. Der Bernstein und die in ihm befindlichen Pflanzenreste der Vorwelt. Berlin, Preussen. 125 pp.

*Beyle, M., 1901. Uber ein altes Torfmoor im hohen Elbufer vor Schulau. Ver. naturw. Unterh. Hamburg 11:

Beyle, M., 1913 ['1912']. Über einige Ablagerungen fossiler Pflanzen der Hamburger Gegend. Mitt. Min.-Geol. Inst. Hamburg. Erster Teil 30(6 Beiheft):83-99.

Beyle, M., 1920. Uber einige Ablagerungen fossiler Pflanzen der Hamburger Gegend. Mitt. Min.-Geol. Inst. Hamburg. Erster Teil 36:33-47.

*Beyle, M., 1924. Uber einige Ablagerungen fossiler Pflanzern in der Hamburger Gegend. Mitt. Min.-Geol. Inst. Hamburg. 6:

*Beyle, M., 1926. Über einige Ablagerungen fossiler Pflanzern in der Hamburger Gegend. Mitt. Min.-Geol. Inst. Hamburg. 8:

*Beyle, M., 1931. Úber ein Torflager am kleinen Pönitzer See im Öslichen Holstein. Mitt. Min.-Geol. Inst. Hamburg. 12:

Birket-Smith, S. J. R., 1977. Fossil insects from Spitsbergen. Acta Arctica 19:1-42.

Blair, K. G., 1923-1924. Some coleopterous remains from the peat-bed at Wolvercote, Oxfordshire. Trans. Ent. Soc. London. 71:558-563. (Bound with Proc. Entomol. Soc. London. 19231924 ['1923'].)

Böcher, J., 1989. Boreal insects in northernmost Greenland: palaeoentomological evidence from the Kap K $\phi$ benhavn Formation (Plio-Pleistocene), Peary Land. Fauna Norv. Ser. B. $36: 37-43$.

Borowiec, L., 1984. Zoogeographical study on Donaciinae of the World (Coleoptera: Chrysomelidae). Polskie Pismo Entomol. Bull, Entomol. Pologne 53:433-518.

*Brauer, F., Redtenbacher, J. \& Ganglbauer, L., 1889. Fossile Insekten aus der Juraformation Ost-Sibiriens. Mém. Acad. Sci. St.-Pétersb. ser. 7, 36(15):1-22.

British Museum (Natural History), 1903. Catalogue of the books, manuscripts, maps, and drawings in the British Museum of Natural History. Order of Trustees. British Museum of Natural History, London.

Brodie, P. B., 1845. A history of the fossil insects in the secondary rocks of England. Accompanied by a particular account of the strata in which they occur, and the circumstances connected with their preservation. John Van Voorst. London. $130 \mathrm{pp}$.

Original, or copies, of the references listed $\left(^{*}\right)$ could not be located. The full citation of about twenty more works could not be confirmed, so they have not been included. 
*Brodie, P. B., 1873. The distribution and correlation of fossil insects, and the supposed occurrence of Lepidoptera and Arachnida in British and foreign strata, chiefly in the secondary rocks, ... being a paper read at the annual meeting of the Warwickshire natural history and archaeological society, held at the museum, Warwick, April 18th, 1873.19 pp. Distr. corr. foss. ins. 16. (Apparently, also in Ann. rep. Warw. nat. hist. arc. soc. 37:12-28.)

Brongniart, A. T., 1827. Succin. In: Cuvier, G. C. (ed.). Dictionnaire des sciences naturelles, dans lequel on traite méthodiquement des différents êtres de la nature, considérés soit en eux-mêmes, d'après l'état actuel de nos connoissances, soit relativement à l'utilité qu'en peuvent retirer la médecine, l'agriculture, le commerce et les arts. Suivi d'une biographie des plus clèbres naturalistes ... Par plusieurs professeurs du Jardin du loi, et des principales écoles de Paris. Strassbourg. Paris. 51:229-240.

Budde, H., 1937. Käfer-Reste aus dem letzten Glacial. Entomol. Blätter 33:378.

Burmeister, C. H. C., 1831. Derselbe über Bernsteininsecten. In: Hünefeld, L. Isis von Oken. Heft VIII.-X. Tafel VII. VII, pp. 11001101 (incorrectly paginated 2000-2001). Brockhaus, Leipzig.

Burmeister, C. H. C., 1832. Kerfe der Urwelt. pp. 632-640. In: Handbuch der Entomologie. $696 \mathrm{pp}$.

Burmeister, C. H. C., 1836. Insects of a former world. pp. 574-581. In: A manual of entomology. Shuckard, W. E. (ed.). E. Churton, London. $654 \mathrm{pp}$.

Carpenter, F. M., 1930. A review of our present knowledge of the geological history of the insects. Psyche 37:15-34.

Carpenter, F. M., 1992. Hexapoda. In: Treatise of Invertebrate Paleontology. Part R. Arthropoda 4(3 and 4). Geol. Soc. Amer. Boulder, CO. $655 \mathrm{pp}$.

Carpenter, F. M. \& Burnham, L., 1985. The geological record of insects. Ann. Rev. Earth Planet. Sci. 13:297-314.

Chagnon, G., 1895. Insectes des âges disparus. Le Naturaliste Canadien. 22:109-110.

Chamisso, A. von, 1824. Untersuchung eines Torfmoores bei Greifswald. Arch. Bergbau Hüttenw. 8:129-139.

Cockerell, T. D. A., 1920a. Fossil arthropods in the British Museum. I. Ann. Nat. Hist. (London) 6:65-72.

Cockerell, T. D. A., 1920b. Fossil arthropods in the British Museum. III. Ann. Nat. Hist. (London) 5:455-463.

Cockerell, T. D. A., 1921 ['1920]. Eocene insects from the Rocky mountains. Proc. U.S. Natl. Mus. 57:233-260.

Cockerell, T. D. A., 1925. British fossil insects. Proc. U.S. Natl. Mus. 49(2119):469-499.

Cockerell, T. D. A., 1926. Some Tertiary fossil insects. Ann. Mag. Nat. Hist. 18:313-324.

Cockerell, T. D. A., 1927. Beetles of the genus Donacia from the Pleistocene of Vancouver Island, British Columbia. Canad. Entomol. 59:303-304.

Cockerell, T. D. A. \& LeVeque, N., 1931. The antiquity of insect structures. Amer. Natur. 65:351-359.

Commission de Stratigraphie. International Geological Congress, 1956. Lexique stratigraphie international. Centre National de la Recherche Scientifique. Paris.

Coope, G. R., 1970. Interpretations of Quaternary insect fossils. Ann. Rev. Entomol. 15:97-120.

Coope, G. R., 1978. Constancy of insect species versus inconstancy of Quaternary environments. Chapter 11. pp. 176-187. In: Mound, L. A. and Waloff, N. (eds.) Diversity of insect faunas. Blackwell Scient. Publ., Oxford. 204 pp.

Coope, G. R., 1979a. Late Cenozoic fossil Coleoptera: Evolution, biogeography, and ecology. Ann. Rev. Ecol. Syst. 10:247-267.

Coope, G. R., 1979b. The Carabidae of the Glacial Refuge in the British Isles and their contribution to the post glacial colonization of Scandinavia and the North Atlantic islands. Chapter 2.43. pp. 407-424. In: Erwin, T. L., Ball, G. E., Whitehead, D. R., and Halpern, A. L. (eds.). Carabid beetles: their evolution, natural history, and classification. W. Junk Publ., The Hague, The Netherlands. $635 \mathrm{pp}$.

Craw, R. C. \& Watt, J. C., 1987. An Upper Cretaceous beetle (Coleoptera) from Hawkes Bay, New Zealand. J. Roy. Soc. New Zealand 17:395-398.

Crowson, R. A., Rolfe, W. D. I., Smart, J., Waterson, C. D., Riley, E. C. \& Wooton, R. J., 1967. Arthropoda: Chelicerata, Pycnogonida, Paleosopus, Myriapoda and Insecta. Chapter 19. pp. 499-534. In: The fossil record: a symposium with documentation, jointly sponsored by the Geological Society of London and the Paleoentomological Association. Part II. Geol. Soc. (London) Part II. $827 \mathrm{pp}$

Crowson, R. A,, 1975. The evolutionary history of Coleoptera, as documented by fossil and comparative evidence. Atti X Congr. Naz. Ital. Entomol. (Sassari, 20-25 May 1974). pp. 47-89.

Crowson, R. A., 1981. Evolutionary history of beetles. Chap. 20. In: The biology of the Coleoptera. Academic Press. London. pp. 658-688.

Crowson, R. A., 1992 ['1991']. The relations of Colcoptera to Cycadales. pp. 13-28. In: Zunino, M., Bellés, X., and Blás, M. (eds.) Advances in Coleopterology. European Association of Coleopterology. 323 pp. Barcelona, Spain.

Curtis, J., 1829. Observations upon a collection of fossil insects discovered near Aix-en-Provence in the summer of 1828, by R. J. Murchinson, Esq. and Charles Lyell, Esq. jun. Edinb. New Philo. Jour. 7:293-297.

Dalrymple, G. B., 1991. The age of the earth. Stanford Univ. Press. $474 \mathrm{pp}$.

Debray, H., 1873. Etude géologique et archéologique de quelques tourbières du Littoral Flammand et du département de la Somme. Mém. Soc. Sci. Agric. Arts Lille 3 ser. 11:433-495.

Debray, H., 1874. Tourbières du Littoral Flamand et du département de la Somme. Bull. Soc. Géol. France 12, 3 ser. 2:46-49.

Debray, H., 1878. Communications diverses, au sujet des tourbières. Ann. Soc. Gol. Nord. 5:125-135.

*Deichmüller, J. V., 1886. Dic Insecten aus dem lithographischen im Dresdener Museum. Mitt. königl. Min.-Geol. Praehist. Mus. Dresden. Heft 7.84 pp.

Elias, S. A., 1982. Paleoenvironmental interpretation of Holocene insect fossils from Northeastern Labrador, Canada. Arctic Alpine Res. 14:311-319.

Elias, S. A., 1985. Paleoenvironmental interpretations of Holocene insect fossil assemblages from four high-altitude sites in the front range, Colorado, U.S.A. Arctic Alpine Res. 17:31-48.

Elias, S. A. 1987. Paleoenvironmental significance of Late Quaternary insect fossils from packrat middens in south-central New Mexico. Southwest. Natur. 32:383-390.

Elias, S. A., 1991. Insects and climate change. BioScience 41:552559.

Elias, S. A., 1992a. Late Quaternary beetle faunas of southwestern Alaska. Evidence of a refugium for mesic and hygrophilous species. Arctic Alpine Res. 24:133-144.

Elias, S. A. 1992b. Later Quaternary zoogeography of the Chihuahuan Desert insect fauna, based on fossil records from packrat middens. Jour. Biogeogr. 19:285-297.

Elias, S. A., Carrara, P. E., Toolin, L. J., and Jull, A. J. T. 1991. Revised age of deglaciation of Lake Emma based on new carbon and macrofossil analyses. Quatern. Res. 36:307-321.

Elias, S. A., Mead, J. I. \& Agenbroad, L. D., 1992. Late Quatcrnary arthropods from the Colorado Plateau, Arizona and Utah. Grcat Basin Naturalist 52:59-67.

Elias, S. A. and Nelson, A. R. 1989. Fossil invertebrate evidence for Late Wisconsin environments at the Lamb Spring Site, Colorado. Plains Anthropol. 34:309-326.

Elias, S. A. and Short, S. K. 1992. Paleoecology of an interglacial peat deposit, Nuyakuk, southwestern Alaska, U.S.A. Géogr. Phys. Quatern. 46:85-96. 
Elias, S. A., Short, S. K., and Clark, P. U. 1986. Paleoenvironmental interpretations of the Late Holocene, Rocky Mountain National Park, Colorado, U.S.A. Rev. Paléobiol. 5:127-142.

Elias, S. A. \& Van Devender, T. R., 1990. Fossil insect evidence for Late Quaternary climatic change in the Big Bend Region, Chihuahuan Desert, Texas. Quartern. Res. 34:249-261.

Elias, S. A. and Van Devender, T. R. 1992. Insect fossil evidence of Late Quaternary environments in the northern Chihuahuan desert of Texas and New Mexico: comparisons with the paleobotanical recod. Southwest. Natur. 37:101-116.

Elias, S. A. and Wilkinson, B. 1983. Lateglacial insect fossil assemblages from Lobsigensee (Swiss Plateau). Studies in the Late Quaternary of Lobsigensee 3. Rev. Paléobiol. 2:189-204.

Ermisch, K., 1942. Airaphilus denticollis, n. sp. (Donacia spec. Helm.). Entomol. Blätter 38:128-129.

Erwin, T. L., 1983. Tropical forests: their richness in Coleoptera and other arthropod species. Coleops Bull. 36:74-82.

Fabricius, I. C., 1775. Systema entomologicae, sistens insectorum classes, ordines, genera, species, adiectis synonymis, locis, descriptionibus, observationibus. Officina Libraria Kortii. Flensburgi et Lipsiae. $832 \mathrm{pp}$.

Fabricius, J. C., 1792. Entomologia systematica emendata et actua, secundum classes, ordines, genera, species adjectis synonymis, locis, observationibus, descriptionibus. Impensis C. G. Proft. Hafniae. Tom. I Pexperiencedars I. 330 pp., Pars. II. 538 pp., Pars. III. 519 pp.

Fairbridge, R. W. \& Jablonski, D., 1979. The encyclopedia of paleontology. Dowden, Hutchinson \& Ross. Stroudsburg, PA. $886 \mathrm{pp}$.

Farrell, B. \& Mitter, C., 1990. Phylogenesis of insect/host plant interactions: Have Phyllobrotica leaf beetles (Chrysomelidae) and the Lamiales diversified in parallel? Evolution 44:1389-1403.

Farell, B. D., Mitter, C. \& Futuyma, D. J., 1992. Diversification at the insect-plant interface. Insights from phylogenetics. BioScience 42:34-42.

Flach, K., 1884. Die Käfer der Unterpleistocänen Ablagerungen bei Hösbach unweit Aschaffenburg. Verhandl. Physikal.-Med. Gessells. Würzburg, n. f. 18:285-297.

Fliche, P., 1875. Sur les lignites quaternaires de Jarville, près de Nancy. Comptes Rendus hebd. séanc. Acad. sci. 80:1233-1236.

Fliche, P., 1876. Faune et flore des tourbières de la Champagne. Comptes Rendus hebd. séanc. Acad. sci. 82:979-982.

Förster, B., 1885. Mitteilungen über das Oberelsässische Tertiär. Tagebl, versamml. Deutsch. Naturf. Aerzt. Strass. 58:379-388.

Förster, B., 1886. Die Gliederung des Sundgauer Tertiärs. Mitth. Comm. Geol. Land. Unter. Elsass. Lothr. Band I. Heft I. pp. $150-177$.

Förster, B., 1889. Vorläufige Mitteilung über die Insekten des 'Plattigen Steinmergels' von Brunstatt. Mitt. Comm. Geol. Elsass.-Lothr. Band II.-Heft I. pp. 102-104.

Förster, B., 1891. Die Insekten des 'Plattigen Steinmergels' von Brunstatt. Alsace-Lorraine. Geologische Landesanstalt. Abhandl. zur Geol. Specialk. von Elsass-Lothringen. 3:335-593.

*Früh., 1885. Beiträge zur Kenntnis des Torfes. Jahrb. k. k. Geol. Reichsanst. 5:

Fujiyama, I., 1980. Late Cenozoic insects from Tokai and Mikawa Districts, Central Japan. Mem. Natl. Sci. Mus. (Tokyo, Japan) 13:21-28. (In Japanese with English summary).

Furth, D. G., 1979 ['1978']. A fossil flea beetle (Coleoptera: Chrysomalidae) from Lake Huleh, Israel. Israel J. Entomol. 12:41-49.

Garry, C. E., Schwert, D. P., Baker, R. C., Kemmis, T. J. Horton, D. G. \& Sullivan, A. E., 1987. Plant and insect remains from the Wisconsinan interstadial/Stadial transition at Wedron, northcentral Illinois. Quatern. Res. 33:387-399.

Garry, C. E., Baker, R. T., Schwert, D. P. \& Schneider, A. F., 1990. Environmental analysis of a Twocreekan-aged beetle
(Coleoptera) assemblage from Kewaunee, Wisconsin. Geol. Soc. Amer. Spec. Pap. 251:57-65.

Germar, E. F., 1813. Insecten in Bernstein eingeschlossen beschrieben aus dem academischen Mineralien-Cabinet zu Italie. Mag. Entomol. 1:11-18.

*Germar, E. F., 1839. Die versteinerten Insekten Solnhofens. Nova acta Acad. Cesareae Leopold.-Carol. Germanicae Naturae Curios. 19:187-222.

Giebel, C. G. A., 1852. Deutschlands Petrefacten. Ein systematisches Verzeichniss aller in Deutschland und den angrenzenden Ländern vorkommenden Petrefacten nebst Angabe der Synonymen und Fundorte. Verlag von Ambrosius Abel. Leip. zig. $706 \mathrm{pp}$.

Giebel, C. G. A., 1856. Insecten und Spinnen der Vorwelt mit steter Berücksichtigung der lebenden Insekten und Spinnen; Monographisch dargestellt. In: Fauna der Vorwelt. II. Band. F. A. Brochaus. Leipzig. 511 pp.

Gingerich, P. D., 1979. Stratophenetic approach to phylogeny reconstruction in vertebrate paleontology. In: Cracaft, J. and Eldredge, N. (eds.). Phylogenetic analysis and paleontology. Proceedings of a symposium entitled 'Phylogenetic models'. North American Paleontological Convention II. Lawrence, Kansas. Aug. 8, 1977. Columbia Univ. Press. New York. $233 \mathrm{pp}$.

Girling, M. A., 1976. Fossil Colcoptera from the Somerset levels: The Abbott's way. Somer. Lev. Pap. 2:28-33.

Girling, M. A., 1977. Fossil insect assemblages from Rowland's tract. Somer. Lev. Pap. 3:51-60.

Girling, M. A., 1978. The application of fossil insect studies to the Somerset levels. Occas. Pap. (Inst. Archeaol.) 3:85-90.

Goecke, H. 1943. Monographie der Schilfkäfer II. Die fossilen Funde und ihre Bestimmung (13. Beitrag zur Kenntnis der Donaciinen.) Nova Acta Leopold. (N. F.) 12:339-380.

Goecke, H., 1960a. Monographie der Schilfkäfer. Die Gattungen und Arten der Donaciinae (Col. Chrys.) und ihre Verbeitung. (18. Beitrag zur Kenntnis der Donaciinen). Entomol. Blätter 56:1-19.

Goecke, H., 1960b. Donaciineen der oligocänen Ablagerungen von Rott. 16. Beitrag zur Kenntnis der Donaccinen (Col., Chrys.). Decheniana 112:279-281.

Göppert, H. R., 1855. Die tertiäre flora von Schossnitz in Schlesien. Heyn'sche Buchhandlung (E. Remer.). Vol. 7.52 pp.

Grande, L., 1980. Paleontology of the Green River Formation, with a review of the fish fauna. Bull. Geol. Surv. Wyoming. 63:1-333.

Gressitt, J. L., 1963. A fossil chrysomelid from the amber of Chiapas, Mexico. J. Paleontol. 37:108-109.

Gressitt, J. L., 1971. A second fossil chrysomelid beetle from the amber of Chiapas, México. In: Studies of fossiliferous amber arthropods of Chiapas, Mexico. Univ. California Publ. Entomol. 63:63-64.

Grimaldi, D. \& Maisey, J., 1990. Introduction. pp. 5-14. In: Grimaldi, D. (ed.) 1990. Insects from the Santana Formation, Lower Cretaceous, of Brazil. Bull. Amer. Mus. Nat. Hist. 195:1195 .

*Gripp, K. \& Beyle, M., 1937. Das Interglacial von Billstedt (Ojendorf). Mitt. Min.-Geol. Inst. Hamburg. 16:

Gyllenhal, L., 1808-1827. Insecta Svecica. Classis I. Coleoptera sive Eleutherata. Tom. I. Pars I-IV. [Pars I. (1808) 572 pp., Pars II. (1810) 660 pp., Pars III. (1813) 730 pp., Pars IV. (1827) 761 pp.]

*Gyllenhal, L., 1817. Anmärkningar rörande ett af Baron de Geer, under namen af Attelabus glaber besrifvit insekt. Stockholm. Acad. Handl. 38:137-141.

Hall, W. E., Van Devender, T. R. \& Olson, C. A., 1988. Late Quaternary arthropod remains from Sonoran desert packrat middens, southwestern Arizona and northwestern Sonora. Quatern. Res. 29:277-293. 
Handlirsch, A., 1906-1908a. Die fossilen Insekten und die Phylogenie der rezenten Formen. Ein Handbuch für Paläontologen und Zoologen. Textband. Vol. I, Text, 1430 pp.; Vol. II, Atlas. Verlag von Wilhelm Engelmann, Leipzig.

Handlirsch, A., 1906-1908b. Die fossilen Insekten und die Phylogenie der rezenten Formen. Ein Handbuch für Paläontologen und Zoologen. Tafelband. Verlag von Wilhelm Engelmann, Leipzig.

Handlirsch, A., 1910. Canadian fossil insects. 5. Insects from the Tertiary Lake deposits of the southern interior of British Columbia, collected by Mr. Laurence M. Lambe, in 1906. Contributions to Canadian Paleontology. Vol. II. Pt. III. pp. 93-129.

Harpe, $P ., 1877$. Sur un gisement de tourbe glaciaire trouvé à Lausanne. Bull. Soc. Vaud. Sci. Nat. $2^{\mathrm{c}}$ S. 14:456-458.

Hartz, N., 1902. Bidrag til Danmarks senglaciale Flora og Fauna. Danmarks geol. Unders申gel. (2 Raekke) 11:1-80.

Hartz, N., 1909. Bidrag til Danmarks tertiaere og diluviale Flora. Danmarks geol. Unders $\phi$ gel. (2 Raekke) 20:1-292.

Hartz, N. \& Milthers, V., 1901. Det senglaciale Ler i Aller $\phi$ d Teglvaersksgrav. Medd. Dansk. geol. Foren. 8:31-60.

Haupt, H., 1950. Die Käfer (Coleoptera) aus der Eozänen Braukohle des Geiseltales. Geologica (Berlin) 6:1-168.

Haupt, H., 1956. Beitrag zur Kenntnis der eozänen Arthropoden-fauna des Geiseltales. Nova Acta Leopold., Halle Deutsche Akademie der Naturforscher (N. S.) ser. 2, 18(128): $1-90$.

Heer, O., 1847-1853. Die Insektenfauna der Tertiärgebilde von Oeningen und von Radoboj in Croatien. I Teil. Verlag Engelmann. Leipzig. Third volume. $229 \mathrm{pp}$.

Heer, O., 1856a. Über die fossilen Insekten von Aix in der Provence. Viert. naturf. geselsch. Zürich 1:1-40.

Heer, O., 1856b. Uber die Insektenfauna von Radoboj. Amtlich. Ber. Versamm. Gesell. deutscher Naturíorsch. Ärtze 32:118-121.

Heer, O., 1858a. Lettre de M. Le Professeur Oswald Heer à Sir Ch. Lyell. Bull. Séances Soc. Vaud. Sci. nat. 5:145-151.

Heer, O., 1858b. Les charbons feuilletés de Durnten et D'Utznach. Arch. sci. phys. et nat., n. p. 2:305-339.

*Heer, O., 1862. Beiträge zur Insektenfauna Oeningens. Coleoptera: Geodephagen, Hydrocanthariden, Clavicornen, Lamellicornen und Buprestiden. Zürich. 90 pp.

Heer, O., 1865. Die Urwelt der Schweiz. Mit sieben landschaftlichen Bildern, elf Tafeln, einen geologischen Ubersichtskarte der Schweiz und zahlreichen in den Text eingendruckten Abbildungen. F. Schulthess. Zürich. $622 \mathrm{pp}$.

Heer, O., 1868a. Die fossile flora der Polarländer enthaltend die in Nordgrönland, auf der Melville-Insel, im Banksland, am Mackensie, in Island und Spitsbergen entdeckten Pflanzen. Flora fossilis arctica. Zürich. Druck und Verlag von Friedrich Schulthess. $192 \mathrm{pp}$.

*Heer, O., 1868b. Flora fossilis arctica. Die fossile flora der Polarländer. 7 Band. Der zweiten Theil der fossilen Flora Grönlands. Verlag Wurster \& Co. 273 pp.

${ }^{*}$ Heer, O., 1869. Flora fossilis alaskana. Flora fossilis von Alaska. Kongl. Svenska Vetenskapsakad. Handl. 8:1-41.

*Heer, O., 1870. Die Miocene Flora und Fauna Spitsbergens. Mit einem Anhang über die diluvialen Ablagerungen Spitsbergens. Kongl. Svenska Vetenskapsakad. Handl. 7(7):1-98.

Heer, O., 1872. Le monde primitif de la Suisse. Traduit de l'allemand par Isaac Demole. Genève et Bâle. 772 pp.

Heer, O., 1876. The primavaeval World of Switzerland. 2 vols. Longmans, Green, and Co., London. Vol. 1. 393 pp. Vol. 2. 324 pp.

Heer, O., 1877. Die Vorweltliche Flora der Schweiz. Flora Fossilis Helvetiae. Verlag von J. Wurster \& Co., Zürich. 182 pp.

Heer, O., 1879. Die Urwelt der Schweiz. Zweite umgearbeite und vermehrte Auflage. Zürich. 713 pp.
Heer, O., 1883a. Die fossile flora der Polarländer enthaltend die in Nordgröndland auf der Melvilleinsel, im Banksland, am Mackensie, in Island und in Spitsbergen entdeckten fossilen Pflanzen. III. Ueber die fossilen Insekten Grönlands. 7 Band. Den zweiten Theil der fossilen Flora Grönlands. Flora fossilis aretica. Verlag von J. Wurster \& Co., Zürich. 275 pp.

Heer, O., 1883b. Die Urwelt der Schweiz. Zeite SubscriptionsAusgabe der zweiten umgearbeiteten und vermehrten Auflage. F. Schulthess. Zürich. $713 \mathrm{pp}$.

Heim, A. \& Gams, H., 1918. Interglaziale Bildungen bei Wildhaus (Kt. St. Gallen). Viertel. Naturf. Gessel. Zürich 63:19-33.

Helm, O., 1896. Beiträge zur Kenntiss der Insecten des Bernsteins. Schr. Nat. Ges. Danzig. (Anlage C.) n. ser. 9:220-231.

Helm, O., 1897. Thierische Einschlüsse im Succinit. Schr. Nat. Ges. Danzig. 9:88-89.

Hennig, W., 1981. Insect phylogeny. J. Wiley \& Sons, Chichester, England. $514 \mathrm{pp}$.

Henriksen, K. L., 1914. Den senglaciale og alluviale Insektfauna i Fems $\phi l y n g$ Mose i Nord Sjaelland. Mindeskrift for Japetus Steenstrup. Kфbenhavn. 35:1-43.

Henriksen, K. L., 1922. Eocene insects from Denmark. Danmarks geologiske unders $\phi$ gelse. II. Raekke. Nr. 37. I Kommission hos C. A. Reitzel. 36 pp.

Henriksen, K. L., 1933. Undersфgelser over Danmark-Skånes kvataere Insektfauna. Vidensk. Meddel. Dansk naturh. Foren. 96:77-355.

Heyden, C. von, 1862. Gliederthiere aus der Braunkohle des Niederrhein's, der Wetterau und der Röhn. Paleontographica 10:62-82.

Heyden, C. von \& Heyden, L. von, 1865. Fossile Insekten aus der Braunkohle von Salzhaussen. Paleontographica 14:31-35.

Heyden, C. von \& Heyden, L. von, 1866. Käfer und Polypen aus der Braunkohle des Siebengebirges. Paleontographica 15:131-156.

Hieke, F. \& Pietrzeniuk, E., 1984. Die Bernstein-Käfer des Museums für Naturkunde, Berlin (Insecta, Coleoptera). Mitt. zool. Mus. Berlin 60:297-326.

Hoganson, J. W. \& Ashworth, A. C., 1992. Fossil beetle evidence for climatic change $18,000-10,000$ years b.p. in south-central Chile. Quatern. Res. 37:101-116.

Hogue, S. M., 1970. Biosystematics of the genus Trirhabda LeConte of America North of Mexico (Chrysomelidae: Coleoptera). Ph. D. Diss. (Univ. Idaho, Moscow, USA). 212 pp.

*Holst, N. O., 1906. De senglaciala vid Toppeladugard. Sver. geol. undersokn. Ser. C 200:

*Holst, N. O., 1908. Efterskörd fran de senglaciala Lagren vid Toppelaguard. Sver. geol. undersokn. Ser. C 210:

Hope, F. W., 1847. XXXXIX. Observations on the fossil insects of Aix in Provence, with descriptions and figures of three species. Trans. Ent. Soc. Lond. 4:250-255.

Hoppe, D. H., 1795. Enumeratio insectorum Elytratorum circa Erlangam indigenarum secundum systema fabricianum observatonibus iconibusque illustrata. $70 \mathrm{pp}$.

Jablonski, D., Flessa, K. W. \& Valentine, J. W., 1985. Biogeography and paleobiology. Paleobiology $11.75-90$.

Jarzembowski, E. A., 1980. Fossil insects from the Bembridge Marls, Palaeogene of the Isle of Wight, southern England. Bull. British Museum (Natural History): Geology (London) 33:237-293.

*Jentzsch, 1910 (probably published in 1913). Bericht über Aufnahmen auf Blatt Schwersenz (Prov. Posen). Jahrb. Preuss. Geol. Landes. 31:

Jessen, K., 1920. Moseunders $\phi$ gelser i det nord $\phi$ stelige Sjælland. Danmarks geol. unders $\varphi$ l. 34:1-268.

* Jessen, K., 1923. En undersoisk Mose i Runsgsted Havn. Danmarks geol. unders $\phi 1.18$ :

Jessen, W., 1932. Käferreste im Tuul von Westerland (Sylt) und die jungterquartäre Schichtenfolge auf den nordfriesischen Inseln. Zentralbl. Min. Geol. Paläontol. Abt. B. 1:311-317. 
Jolivet, P., 1970. Donaciinae. Coleopterorum Catalogus. Suplementa. Junk Pubs. Pars 51(2). Editio Seconda. 71 pp.

Kenward, H. K., 1978. The value of insect remains as evidence of ecological conditions in archeological sites. Occas. Pap. (Inst. Archeol.) 3:25-38.

Kimoto, S., 1981. Studies on the Japanese Donaciinae (Coleoptera: Chrysomelidae). 1. Data of distribution and biology obtained from survey in 1979 and 1980. Fossil Insect Research Group for Nojiri-ko Excavation. Bull. Osaka Mus. Nat. Hist. no. 34:27-46. (In Japanese, abstract in English)

Kiselev, S. V., 1981. Pozdnekainozoiskie zhestkokrylye SeveroVostoka Sibiri. Moskva: 'Nauka'. Akademiia nauk SSSR. Paleontologicheskii institut. Nauchnyi sovet po probleme 'Puti i zakonomernosti istoricheskogo razvitiia zhivotnykh $i$ rastitel'nykh organizmov.' 116 pp. (In Cyrilic)

Klebs, R., 1910. Über Bernstcinschlüsse im allgemeinen und die Coleopteren meiner Bernsteinsammlung. In: Schriften der Physikalish-ökonomischen Gesellschaft zu Königsberg in Pr. Im Kommission bei Wilh. Koch. pp. 217-242.

Kolbe, H. J., 1894. Über fossile Reste von Coleopteren aus einem alten Torflager (Schmierkohle) bei Gr. Räschen in her NiederLausitz. Sitz.-Berich. Gesell. Naturforsch. Frend. Berl. 1894:236238.

Kolbe, H. J., 1933. Fossile Coleopteren aus praeglazialer und spätglazialer Zeit Süd-Schwedens. Entomol. Medd. 18:209-214.

*Kolumbe, E. \& Beyle, M., 1938. Die Bohlwege im Wittmoor (Holstein) und ihre Stellungen im Pollendiagramm. In: Carsten, R. H. (ed.) Zwischen Elbe und Skagerrak. Hamburg, Germany. p. 53-54.

Korschefsky, R., 1939. Abbildungen und Bemerkungen zu vier Schaufuss'schen Coleopteren aus dem deutschen Bernstein. Arbeit. Morphol. Taxonom. Entomol. Berlin-Dahlem 6:11-12.

Kühne, W. G. \& Schlüter, T,, 1985. A fair deal for the Devonian Arthropoda fauna of Rhynie. Entomol. Gen. 11:91-96.

Kukalová-Peck, J., 1991. Fossil history and the evolution of hexapod structures. In, The insects of Australia: a textbook for students and research workers. Chap. 6, pp. 141-179. Div Entomol. Commonw. Sci. Industr. Res. Org. 2nd. ed. Ithaca, NY. Cornell Univ. Press. Vol. 1. 542 pp.

*Kunze, G., 1818. Beiträge zur Monographie der Rohrkäfer. Neue Schrif. Naturforsch. Gesell. Halle. 2:1-56.

*Kurck, C., 1910. Arkeologiska och växgeografiska öfver skanska torfmossar. Ymer. Tidsskr. Svensk. Sällsk. Antrop. Geogr. 30:

Kurck, C., 1917. Den forntida Utbredningen af kärrsköldpaddan, Emys orbicularis (Lin.). I. Sverige, Danmark och angränsande länder mit einer zusammenfassung in deutscher Sprache. Lunds Univ. Årsskr. N. F. Avd. 2 13:1-128.

Labandeira, C. C. and Sepkoski Jr, J. J. 1993. Insect diversity in the fossil record. Science 263:310-315.

*Laicharting, J. N. Edlen von, 1781-1784. Verzeichniss und Beschreibung der Tyroler Insekten. Zurich. 2 Band.

Lapouge, G. de, 1902. Degré d'évolution du genre Carabus à l'époque du pléistocène moyen. Bull. soc. sci. méd. Ouest. (Rennes) 11:548-566.

Larsson, S. G., 1978. Baltic amber - a paleobilogical study. Entomonograph. Vol. 1. Scandinavian Science Press, Ltd. Klampenborg, Denmark. 192 pp.

Latreille, M., 1819. Encyclopédie méthodique histoire naturelle entomologique, ou histoire naturelle des Crustacés, des Arachnides et des insectes. Tome neuvième. $828 \mathrm{pp}$.

Lea, P. D., Elias, S. A. and Short, S. K. 1991. Stratigraphy and paleoenvironments of Pleistocene nonglacial deposits in the southern Hushagak Lowland, southwestern Alaska, U.S.A. Arctic Alpine Res. 23:375-391.

Lesne, P., 1918a. Le faune entomologique subfossile des tourbières sous-marines de Belle-Ile. Comptes Rendus hebd. séanc. Acad. sci. $167: 538-540$.
Lesne, P., 1918b. Insectes subfossiles des tourbières sous-marines de Belle-Ile. Bull. Mus. Natl. Hist. nat. 24:397-402.

Lesne, P., 1925. Sur la faune des alluvions tourbeuses de la vallée de la Seine au sud de Paris. Comptes Rendus hebd. séanc. Acad. sci. 180:947-949.

Lewis, S. E. \& Carroll, M. A., 1991. Coleopterous egg deposition on alder leaves from the Klondike mountain formation (Middle Eocene), northeastern Washington. J. Paleontol. 65:334-335.

Linné, C. von, 1758. Caroli Linnaei Systema naturae: Regnum animale. A photographic edition facsimile of the first volume of the tenth edition (1758). Trustees, British Museum (London). 823 pp.

Lomnicki, A. M., 1894. Fauna pleistocenica insectorum Boryslaviensium. Pleistocénskie owady z Borystawia. Mus. Imenia Dzieduszyckich 4:1-127.

Lopatin, I. K., 1984. Leaf beetles (Chrysomelidae) of Central Asia and Kazakhtan. Amerind Publ. Co. New Delhi, India. 416 pp.

Lortet, M. D. \& Chantre, M. E., 1872. Etudes paléontologiques dans le bassin du Rhône Période Quaternaire. Arch. mus. hist. nat. Lyon. 1:59-130.

Luttrell, G. L. W., Hubert, M. L. \& Jussen, V. M., 1986. Lexicon of new formal geologic names of the United States, 1976-1980. Washington, D. C. $191 \mathrm{pp}$.

Lutz, H., 1990. Systematische und palökologische Untersuchungen an Insekten aus dem Mittel-Eozän der Grube Messel bei Darmstadt. Courier Forsch. Senckenberg 124:1-165. Fossitfundstelle Messel Nr. 90. (Originally presented as Dissertation. Technische Hochschule Darmstadt. Fachbereich Biologie. Kennziffer D 17. Darmstadt. 1988.)

Lyell, C., 1840. On the Boulder Formation or Drift, and associated freshwater deposits composing the mud cliffs of eastern Norfolk. Proc. Geol. Soc. London 3:171-179.

Malfatti, G., 1881. Bibliografia degli insetti fossili italiani finora conosciuti. Atti. soc. ital. sci. nat. 24:89-100.

Martynov, A. K., 1926. To the knowledge of fossil insects from Jurassic beds in Turkestan. 5. On some interesting Coleoptera. Annuaire Société Paléontologique Russie 5:1-38. (In Cyrilic with English summary)

*Martynov, A. V., 1929. [Fossi] insects from tertiary deposits in Ashutas, Zaisan district, S. Siberia.] Trudy Geologicheskogo Muzeia Akademi Nauk SSSR. 5:173-216. (In Cyrilic with Eng. lish summary)

Martynov, A. V., 1935. [Note on the fossil insects from Mesozoic deposits in Cheliabinsk district.] Trudy Paleozoologicheskogo Instituta Akademii Nauk SSSR. 4:37-48. (In Cyrilic with English summary)

Martynova, O. M., 1961. Paleoentomology. Ann. Rev. Entomol. 6:285-293.

May, R. M,, 1990. How many species? Phil. Trans. Roy. Soc. London. (ser. B) 330:293-304.

Medvedev, L. N., 1968. Leaf beetles from the Jurassic of Karataw. Akad. Medit. Nauk. SSSR Otd. biol. (Moskva) p. 155-165.

Menge, A., 1856. 1. Lebenszeichen vorweltlicher, im Bernstein eingeschlossener Thiere. In, Programm womit zu der am Montag den 17. März 1856 von $8 \mathrm{I} / 2 \mathrm{Uhr}$ Vorm. und $21 / 2 \mathrm{Uhr}$ Nachm.an stattfindenden öflentlichen Püfung der Schüler der Petrischule ergebenst einladet. Danzig.

Merritt, R. W. \& Cummins, K. W. (eds.), 1984. An introduction to the aquatic insects of North America. 2nd. ed. Kendall/Hunt Publ. Co. Dubuque. Iowa. $441 \mathrm{pp}$.

*Meunier, F., 1898b. Observations sur quelques Insectes du Corallier de la Bavière. Zeitschr. Entomol. 3:

Meunier, F., 1898c. Les insectes des temps secondaires. Revuc critique des fossiles du Musée paléontologique de Munich. Arch. Mus. Teyler (sér. 2) 6:85-148.

*Meunier, F., 1898d. Description de quelques Coléoptères de l'Oligocène d'Armissan (Aude). Ann. Soc. Scient. Bruxelles 22:113-115. 
Meunier, F., 1900. Sur des élytres de Coléoptères de la tourbe préglaciaire de Lauenburg (Elbe). Bull. Soc. Ent. Fr. 1900:166167.

Meunier, F, 1901. Uber einige Coleopteren-Flügeldecken aus der präglacialen Braunkohle und dem interglacialen Torflager von Lauenburg (Elbe). Jahrb. Preuss. Geol. Landesant. 21:31-38.

*Meunier, F., 1918. Neue Beiträge über die fossilen Insekten aus der Braunkohle von Rott (Aquitanien) im Siebengebirge (Rheinpreussen). Jahrb. koening. Preuss. Geol. Landes. 39:141-153.

Meunier, S., 1884. Traité de Paléontologie pratique gisement et description des animaux et des végétaux fossiles de la France indication de localitées fossilifères, etc. J. Rothchild (ed.) Paris. $495 \mathrm{pp}$.

Monrós, F., 1954. Revision of the chrysomelid subfamily Aulacoscelinae. Bull. Mus. Comp. Zool. 112:320-360.

Moore, R. C., Lalicker, C. G. \& Fischer, A. G., 1952. Invertebrate Fossils. McGraw-Hill Book Co., Inc., New York. 1st. ed. 766 pp.

Morgan, A. V., Morgan, A., Nelson, R. E. \& Pilny, J. J., 1986. Current status of knowledge on the past and present distribution of the genus Blethisa (Coleoptera: Carabidae) in North America. Coleops Bull. 40:105-115.

Morell, V., 1992. 30-million-year-old DNA boosts an emerging field. Science 257:1860-1862.

Mortillet, G. de, 1850. Description d'une nouvelle espèce de coléoptère fossile (Donacia Genin) trouvé dans les lignites de Sonnaz. Arch. sci. phys. et nat. 15:78-79. (Also in Bull. Hist. Nat. Savoie 1850:135 and Les Alpes 1850, no. 5.)

Mulvihill, J., 1982. User guide to the bibliography and index to geology. The American Geological Institute. Leesburg Pike, VA. $160 \mathrm{pp}$.

Nasarow, V. I., 1984. Reconstruction of landscapes of Belorussia by paleontological data (Holocene). Trudy paleontologicheskogo instituta Akademii Nauk SSSR. 205:1-104. (In Cyrilic)

*Nehring, A., 1895. Uber neue Funde von Klinge bei Cottbus. Naturw. Wochenschr. 10:

Nel, A., 1988. Deux Dicladispa fossiles des gisements de diatomites néogènes de Bes-Konak (Turquie) et de Murat (France) (Coleoptera, Chrysomelidae, Hispinae). Geobios 21:369-374.

Newberry, E., 1795. A natural history of fishes, and of reptiles, insects, waters, earths, fossils, minerals, and vegetables. Compiled from the best authorities, and illustrated by a great variety of copper plates, comprising near one hundred figures. London. $208 \mathrm{pp}$.

Nikritin, L. M. \& Ponomarenko, A. C., 1992 ['1991']. Fossil Coleoptera of the USSR: their evolution and distribution. pp. 29-33. In: Zunino, M., Bellés, X., and Blás, M. (eds.) Advances in Coleopterology. European Association of Coleopterology. Barcelona, Spain. 323 pp.

Olivier, M., 1791. Encyclopédie méthodique ou histoire naturelle des insectes. Tome 50. Paris, France. 795 pp.

Oppenheim, P., 1887-1888. Die Insectenwelt des lithographischen Schiefers in Bayern. Palaeontographica. Beitr. Naturg. Vorz. Sttut. 34:215-247.

Ornduff, R., 1974. An introduction to California plant life. Univ. California Press. Berkeley. 152 pp.

Ornduff, R., 1991. Size classes, reproductive behavior, and insect associates of Cycas media (Cycadaceae) in Australia. Bot. Gaz. 152:203-207.

Oustalet, M. E., 1874. Recherches sur les insectes fossiles des terrains Tertiaires de la France. 12 Partie. Insectes Fossiles d'Aix en Provence. Ann. sci. géol. 5:319-347.

Pant, D. D., 1973. Cycads and the Cycadales. Central Book Depot. Alahabad, India. $255 \mathrm{pp}$.

Pant, D. D., 1988 (1987). The fossil history and phylogeny of the Cycadales. Geophytology 17:125-162.

Panzer, G. W. F., 1795. Entomologia Germanica. Exhibens insecta per Germaniam indigena secundum classes, ordines, genera, species, adiectis synonymis, locis, observationibus. I. Eleuterata. $370 \mathrm{pp}$. In: Deutschlands Insectenfauna oder Entomologisches Taschenbuch für das Jahr 1795 . Nürnberg. $370 \mathrm{pp}$.

Pawlowski, J., Kúska, A. \& Warchalowski, A., 1987. Ecological interpretation of the beetle assemblages (Coleoptera faunal units) from the 'Kluki 74' Holocene profile. Preliminary results. Acta Paleobot. 27:223-226.

Paykull, G. von, 1800. Fauna Suecica. Insecta. Upsaliae. Tomus I. 358 pp., Tomus II. 234 pp., Tomus III. 459 pp.

Pearl, R. M., 1951. Guide to geologic literature. McGraw-Hill Book Co., Inc. New York, NY. 239 pp.

Pearson, R. G., 1962. The Coleoptera from a late-glacial deposit at St. Bees, West Cumberland. J. Anim. Ecol. 31:129-150.

Petiver, J., 1764. Jacobi Petiveri Opera, historiam naturalem spectantia; or, Gazophylacium. Containing several 1000 figures of birds, beasts, reptiles, insects, fish, beetles, moths, flies, shells, corals, fossils, minerals, stones, fungusses, mosses, herbs, plants \&c. from all nations, on 156 copper-plates, with Latin and English names. The shells, \&c. have English, Latin, and native names. London. J. Millan, nr. Whiteball. 2 vols.

Phillips, J., 1871. Geology of Oxford and the valley of the Thames. Clarendon Press. Oxford, England. 523 pp.

Pilny, J. J. \& Morgan, A. V., 1987. Paleoentomology and paleoecology of a possible Sangamonian site near Innerkip, Ontario Quatern. Res. 28:157-174.

Piton, L., 1939. Note complémentaire sur les fossiles insectes des cinérites Pliocènes du Lac Chambon (Puy-de-Dôme). Rev. sci. nat. Auvergne 5:102-108.

Piton, L., 1940. Paléontologie du gisement ćocène de Menat (Puy-de-Dôme) (flore et faune) (première thèse). Vallier. Clermont-Ferrand. $303 \mathrm{pp}$.

Piton, L. \& Théobald, N., 1935. Le faune entomologique des gisements Mio-Pliocènes du Massif Central, Rev. Sci. Nat. Auvergne 1:65-104.

Poinar, G. O. Jr., 1992. Life in amber. Stanford Univ. Press. Palo Alto, CA. 350 pp.

Polne, P. \& Coope, G. R., 1990. Lateglacial and Early Flandrian Coleoptera from La Taphanel, Massif Central. France: climatic and ecological interpretations. J. Quatern. Sci. 5:235-249.

Poppius, B., 1911. Beiträge zur postglazialen Einwanderung der Käfer-Fauna Finnlands. Acta Soc. Fauna Flora fennica. 34:1-59.

Ponomarenko, A. G. \& Schultz, O., 1988. Typen der GeologischPaläontologischen Abteilung: Fossile Insekten. Selbstverlag Naturhistorisches Museum Wien. Kataloge der wissenschaftlichen Sammlung des Naturhistorischen Museums in Wien. Paläozoologie. Bd. 6. Heft $1.39 \mathrm{pp}$.

Porter, R., 1983. The earth sciences. An annotated bibliography. Garland Publ., Inc. New York, NY. 1901 pp.

Poulton, E. B., 1923. Fragments of beetles from a Pleistocene peat-bed at Wolvercote, near Oxford. Proc. entomol. Soc. (London) 1923-1924:15-17. (Bound with Proc. Entomol. Soc. London. 1923-1924 ['1923'])

Puni, N., 1881. In, Seduta del 29 maggio 1881. Presidenza del Presidente comm. Prof. E. Cornalia. F. Sordelli, Secretary. Atti. Soc. Ital. Sci. Nat. 24:53-54.

Quedenfeldt, G., 1885. Copal-Insecten aus Africa. Berl. Entomol. Zeitschr. 29:363-365.

Quiel, G., 1909. Zwei neue Coleopteren aus dem baltischen Bernstein. (Eocän bezw. unteres Oligocän. Berl. Entomol. Zeitschr. 54:49-52.

Quiel, G., 1911. ['1910'] Bemerkungen über Coleopteren aus dem baltischen. Berl. Entomol. Zeitschr. 55:181-192.

Reid, C., 1890. The Pliocene deposits of Britain. Mem. Geol. Surv. United Kingdom. London. $326 \mathrm{pp}$.

Richardson, J., 1837. Part the fourth and last. The insects. In: Fauna Boreali-Americana; or the Zoology of the Northern 
Parts of British America: containing descriptions of the objects of natural history collected on the late northern land expedition under command of Cpt. Sir John Franklin, R. N. Norwich. 325 pp.

Robert, E., 1838. [Letter read by M. Cordier on 15 January 1838. .] Bull. Soc. Géol. France. 9:114-118.

Rohdendorf, B. B., 1956. Les insectes Paléozoïques de la Sibérie du sud. Entomol. Obozrenie (Rev. Entomol. URSS) 35:611-619. (In Cyrilic with French summary)

Rohdendorf, B. B., 1957. Paleoentomologicheskie issledovaniia v SSSR. [Paleo-entomological research in the U.S.S.R.]. Moskva, Izd-vo. Akademii nauk SSSR. Paleontologicheskii institut (Akademiia nauk SSSR) Trudy. t. 66. 99 pp. (In Cyrilic)

Rohdendorf, B. B., 1958. Les insectes Paléozoïques de la Sibérie. pp. 853-859. In: Proc. Internt. Congr. Entomol., 10th Congr. Montreal (1956).

*Rohdendorf, B. B. (ed.), 1962. Arthropoda-Tracheata and Chelicerata. In: Textbook of Paleontology. A textbook for the paleontologists and geologists of the USSR. Academy of Sciences of the USSR. Moscow. Vol. 9. pp. 1-561.

Rohdendorf, B. B., 1968. Iurskie nasekomye Karatau Moskva: Izd-vo 'Nauka'. Akademiia nauk SSSR. Otdelenie obshchei biologii. Nauchnyi sovet po probleme 'Puti i zakonomernosti istoricheskogo razvitiia zhivotnykh i rastitel'nykh organizmov.' (Akademiia nauk SSSR). 252 pp. (In Cyrilic)

Rohdendorf, B. B., Zherikhin, V. V. \& Rasnitsyn, A. P., 1980. Istoricheskoe razvitie klassa nasekomykh Moskva: Nauka. Trudy Paleontologicheskogo instituta Akademiia nauk SSSR. T. 175.269 pp. (In Cyrilic)

Rostrup, E., 1859. Beskrivelse af 'Gallemosen' paa Lolland. Vidensk. Meddel. Dansk. naturh. Foren. 10:121-126.

Royal Society of London, 1867-1921. Catalogue of Scientific Papers. G. E. Eyre and W. Spottiswoode, London.

Schäff, H., 1892. Úber Insektenreste aus dem Torflager von Kinge. Sitz.-Berich. Gesell, Naturforsch. Frend. Berl. 1892;8-11.

Schawaller, W., 1986. Fossil beetles from Miocene sediments of the Randeck Maar in southwest Germany (Insecta: Coleoptera). Stutt. Beitr. Naturk. (ser. B) 126:1-9.

Schaufuss, C., 1892. Preussens Bernstein-Käfer. Neue Formen aus der Helm'chen Sammlung im Danziger Provinzialmuseum, Berlin. Berl. Entomol. Zeitschr. 36:53-64.

Schlechtendal, D. H. R. von, 1893. Beitriage zur Kenntis Fossiler Insekten aus dem Braunkohlengebirge von Rott am Siebengebirge. Abhandl. Naturforsch. Gesell. Halle 20:199-228.

*Schlee, D., 1986. Der Bernsteinwald. Staatliches Museum für Naturkunde. 15 pp.

Schmitt, M., 1988. The Criocerinae: biology, phylogeny and evolution. Chapter 28. pp. 476-495. In: Jolivet, P., Petitpierre, E. and Hsiao, T. H. (eds.). Biology of Chrysomelidae. Kluwer Academic Publ. Dordrecht. The Netherlands.

Scholz, R., 1934. Plateumaris sericea L. als Uberreste im Insektentorf. Entomol. Blätter 30:88.

Schneider, E. L. \& Moore, L. A., 1977. Morphological studies on the Nymphaeaceae VII. The floral biology of Nuphar lutea subsp. macrophylla. Brittonia 29:88-99.

Schwert, D. P., 1992. Faunal transitions in response to an ice age: the late Wisconsinan record of Coleoptera in the north-central United States. Coleops Bull 46:68-94.

Schwert, D. P. \& Ashworth, A. C., 1988. Late Quaternary history of the northern beetle fauna of North America: a synthesis of fossil and distributional evidence. pp. 93-107. In: Downes, J. A. and Kavanaugh, D. H. (eds.). Origins of the North America Insect fauna. Mem. Entomol. Soc. Canada 144:1-168.

Schwert, D. P. \& Ashworth, A. C., 1990. Ice Age beetles. Natural History 1990(1):10-14.

Schwert, D. P. \& Morgan, A. V., 1980. Paleoenvironmental implications of a late glacial insect assemblage from northwestern New York. Quartern. Res. 13:93-110.
Scudder, S. H., 1876. Fossil Coleoptera from the Rocky Mountain tertiaries. Bull. U. S. Geol. Surv. Terr. 2:77-87.

*Scudder, S. H., 1878. An account of some insects of unusual interest from the Tertiary rocks of Colorado and Wyoming. Bull. U.S. Geol. Georgr. Surv. Terr. 4:519-543.

*Scudder, S. H., 1879. Appendix A. The fossil insects collected in 1877 by Mr. G. M. Dawson, in the interior British Columbia. Report of Progress of the Geological Survey of Canada 1877 1878. B:176-185.

Scudder, S. H., 1881. The Tertiary lake-basin at Florissant, Colorado between South and Hayden Parks. Bull. U.S. Geol. Surv. Terr. 6:279-300.

Scudder, S. H., 1882 A bibliography of fossil insects. Bibliographical Contributions (Harvard College) 13:1-47.

Scudder, S. H., 1885. Systematische Übersicht der fossilen Myriapoden, Arachnoiden, und Insekten. p. 721-831. In: K. A. von Zittel. Handbuch der Paläontologie. Abtheilung I. Palæozoologie. II. Band Mollusca und Arthropoda. Oldenbourg. München. $893 \mathrm{pp}$.

Scudder, S. H., 1887. ['1886']. Systematic review of our present knowledge of fossil insects, including myriapods and arachnids. United States Geological Survey. Government Printing Office. Washington [D.C.]. Bull. U.S. Geol. Surv. V. no. 31. 1-128 pp.

Scudder, S. H., 1890a. The fossil insects of North America: with notes on some European species. Vol. I. The pretertiary insects. United States. Department of the Interior. Report of the United States Geological Survey of the Territories. $455 \mathrm{pp}$.

Scudder, S. H., 1890b. The fossil insects of North America, with notes on some European species. Vol. II. The Tertiary insects of North America. Report of the United States Geological Survey of the Territories. Dept. Interior. Washington Printing Office. Macmillan and Co., New York. Vol. XIII. 734 pp.

Scudder, S. H., 1890c. A classed and annotated bibliography of fossil insects. 2 ed. Bull. U.S. Geol. Surv. 69:1-101.

Scudder, S. H., 1891. Index to the known fossil insects of the World, including myriapods and arachnids. Bull. U.S. Geol. Surv. no. 71.744 pp. Government Printing Office. Washington [D.C.].

Scudder, S. H., 1893. Tertiary rhynchophorous Coleoptera of the United States. Monogr. U.S. Geol. Surv. 21:1-206.

Scudder, S. H., 1895a. The Miocene insect-fauna of Oeningen, Baden. Geol. Mag. n.s. 2:116-122.

Scudder, S. H., 1895b. 2. The Coleoptera hitherto found fossil in Canada. Contributions to Canadian palaeontology. Vol. II. Pt. II. Canadian fossil insects. $56 \mathrm{pp}$. (According to Wickham, 1920, this is also dated 1892-93.)

Scudder, S. H., 1898. The Pleistocene beetles of Fort Rivers, Massachusetts. pp. 745-746. In: Geology of Old Hampshire County, Massachusetts comprising Franklin, Hampshire, and Hampden Counties. Emerson, B. J. Monogr. U.S. Geol. Surv. 29:1-782.

Scudder, S. H., 1900. Adephagous and clavicorn Coleoptera from the Tertiary deposits at Florissant, Colorado with descriptions of a few other forms and systematic list of the non-rhynchophorous Tertiary Coleoptera of North America. Monogr. U.S. Geol. Surv. 40:1-145.

Seeno, T. N. \& Wilcox, J. A., 1982. Leaf beetle genera (Coleoptera: Chrysomelidae). Entomography 1:1-221.

*Sendel, N., 1742. Historia succinorum corpora aliena involventium et naturae opere pictorum et caelatorum, ex Regiis Augustorum cimeliis Dresdae conditis aeri insculptorum conscripta, a Nathanaele Sendelio. Lipsiae, apud IO.Fridericum Gleditschium. viii, [2], 328 pp.

*Serres, P. M. T, de, 1827. Notice sur les cavernes à ossements fossiles des carrières de calcaire grossier, situées aux environs de Lunnel Vieil dans le département de l'Hérault. Mém. Soc. Linn. Paris 5:442-464. 
Serres, M., 1829. Géognosie des Terrains Tertiaires. Tableau des Principaux animaux invertébrés des terrains marins Tertiaires du midi de la France. Paris. 277 pp.

Short, S. K. and Elias, S. A. 1987. New pollen and beetle analyses at the Mary Jane site, Colorado: Evidence for lata glacial tundra conditions. Geol. Soc. Amer. Bull, 98:540-548.

Short, S. K., Elias, S. A., Waythomas, C. F. and Williams, N. E. 1992. Fossil pollen and insect evidence for postglacial environmental conditions, Nushagak and Holitna lowland regions, southwest Alaska. Arctic 45:381-392.

Sordelli, F., 1882a. Note sopra alcuni insetti fossili di Lombordia (1). Bull. soc. Ent. Ital. 14:224-235.

${ }^{*}$ Sordelli, F., 1882b. Descrizione di alcuni avanzi vegetali delle argille plioceniche Lombarde, collaggiunta di un elenco deille piente fossili finora conosciute in Lombardia. Atti. Soc. Ital. 16:350-429.

Spahr, U., 1981a. Bibliographie der Bernstein - und Kopal-Käfer (Coleoptera). Stutt. Beitr. Naturk. (ser. B) 72:1-21.

Spahr, U., 1981b. Systematischer Katalog der Bernstein - und Kopal-Käfer (Coleoptera). Stutt. Beitr. Naturk. (ser. B) 80:1107.

Starratt, S. W., 1987. Micropaleontology, paleolimnology, and biochronology of middle Miocene lacustrine and nearshore facies belonging to the 'Esmeralda' Formation in Stewart Valley, west Central Nevada. Geol. Soc. Amer. Abstracts with Programs 19:336.

Stevens, P. F., 1980. Evolutionary polarity of character states. Ann. Rev. Ecol, Syst. 11:33-58.

Stevenson, D. W., 1992. A formal classification of the extant cycads. Brittonia 44:220-223.

Strong, D. R., Lawton, J. H. \& Southwood, R., 1984. Insects on plants. Community patterns and mechanisms. Harvard Univ. Press. 313 pp.

Studer, T., 1920-1922. Die Fauna der Schieferkohlen von Gondiswil-Zell. Ecl. geol. Helvetiae 16:128-130.

Sustrac, G., 1984. La bibliothèque de Géologie bibliographie fondamentale thémathique et régionale en sciences de la terre. Bureau des recherches gélogiques et minières. Sér. Doc. BRGM. no. 69. Orléans, France. $192 \mathrm{pp}$.

Suzuki, K., 1985. Phylogenetic relationships among higher taxa of the family Chrysomelidae. Chrysomela 12:13-14.

Théobald, N., 1937. Les insectes fossiles des terrains oligocènes de France. Mém. Soc. Sci. Nancy. $473 \mathrm{pp}$.

Uhmann, E., 1939. Hispinen aus baltischem Bernstein. BernsteinForsch. 4:18-22.

United States Geological Survey, [1955.] A catalog of the Cambrian fossils of the World [as recorded through 1954. Washington, D.C.] General Service Administration. Microfilms.

United States Geological Survey, [no date a] Mesozoic species catalog. North and Central America, including the Caribbean Islands. General Service Administration. Microfilms.

United States Geological Survey, [no date b] Mesozoic species catalog. South America. General Service Administration. Microfilms.

Villiers, A., 1979. Initiation à l'entomologie. Société nouvelle des éditions Boubée, Paris. Vol. 2. $101 \mathrm{pp}$.

Ward, S., 1776. A modern system of natural history: containing accurate descriptions, and faithful histories, of animals, vegetables, and minerals. Together with their properties, and various uses in medicine, mechanics, manufactures, \&c. illustrated with a great variety of copper-plates, accurately drawn from nature, and beautifully engraved. Twelve Volumes. Vol. 11: Natural history of reptiles and insects. $178 \mathrm{pp}$. Vol. 12: Natural history of waters, earths, fossils, minerals and vegetables. $171 \mathrm{pp} . \mathrm{F}$. Newbery, London.

Ward, D. E., Wheeler, M. W. \& Bier, Jr., R. T. A., 1981. Geologic reference sources. A subject and regional bibliography of pub- lications and maps in the geological sciences. Scarecrow Press, Inc. NJ. $560 \mathrm{pp}$.

*Westergard, A. H., 1912. Beskrifning till kartbladt Trelleborg. Sver. geol. Unders $\phi$. ser. Aa (146):

Westwood, J. O., 1845. Introductory remarks. In: Brodie, P. B. A history of the fossil insects in the secondary rocks of England. Accompanied by a particular account of the strata in which they occur, and the circumstances connected with their preservation. John Van Voorst. London. $130 \mathrm{pp}$.

Westwood, J. O., 1854. Contributions to fossil entomology. Quart. Jour. Geol. Soc. London 10:378-396.

Weyenbergh Jr., H. (same as Weijenbergh, H.), 1869a. Sur les insectes fossiles du calcaire lithographique de la Bavière, qui se trouvent au Musée Teyler. Arch. Musée Teyler (ser. 1) 2:247294.

Weijenbergh, H. (same as Weyenbergh, H.), 1869b. Prodromus en algemeene beschouwing der Fossiele Insekten van Beijeren. Tijdschr. v. Entomol. Uit. Neder. Ent. Ver. 2 Ser. 4:230-248.

*Weyenbergh, H., 1874. Enumération systématique des espèces qui forment la faune entomologique de la période mésozoïque de la Bavière; en même temps Supplément du Catalogue de la collection paléontologique du Musée Teyler. In: Varia Zoologica et Paleontologica, Periód. Zool. Org. Soc. Entomol. Argent. 1:17111. Buenos Aires.

Whalley, P. E. S., 1985. The systematics and palaeogeography of the Lower Jurassic insects of Dorset, England. Bulletin of the British Museum (Natural History). Geology series. 39:107-189.

Whitehead, A. H., 1918/21 (1920). More about 'moorlog'. - A peaty deposit from the Dogger Bank in the North Sea. Essex Natur. 19:242-250.

Whitehead, A. H. \& Goodchild, H. H., 1909/11 (1909). Some notes on 'moorlog', a peaty deposit from the Dogger Bank in the North Sea. Essex Natur. 16:51-60.

Whitehead, P. F., 1989. Changing environments and Coleoptera faunas from Aston Mill, Worcestershire, England. Entomol. Month. Mag. 125:187-198.

Wickham, H. F., 1910. New fossil Coleoptera from Florissant, with notes on some already described. Amer. J. Science (ser. 4) 29:47-51.

Wickham, H. F., 1911. Fossil Coleoptera from Florissant, with descriptions of several new species. Bull. Amer. Mus. Nat. Hist. 30:53-69.

Wickham, H. F., 1912. A report on some recent collections of fossil Coleoptera from the Miocene shales of Florissant. Bull. Univ. Iowa Lab. Nat. Hist. 6(3):3-38.

Wickham, H. F., 1913a. The Princeton collection of fossil beeties from Florissant. Ann. Ent. Soc. Amer. 6:359-366.

Wickham, H. F., 1913b. Fossil Coleoptera from Florissant in the United States National Museum. Proc. U.S. Natl. Mus. 45:283303.

Wickham, H. F., 1913c. Fossil Coleoptera from Wilson ranch. Bull. Lab. Nat. Hist. State University Iowa 6:3-38.

Wickham, H. F., 1914a. Twenty new Coleoptera from Florissant. Trans. Amer. Entomol. Soc. 6:257-269.

Wickham, H. F., 1914b. New Miocene Coleoptera from Florissant. Bull. Mus. Comp. Zool. (Harvard Coll.) 58:422-494.

Wickham, H. F., 1917. Fossil beetles from Sangamon Peat. Amer. J. Sci. 44:137-145.

Wickham, H. F., 1920. Catalogue of the North American Coleoptera described as fossils. pp. 349-365. In: Leng, C. W. Catalogue of the Coleoptera of America, north of Mexico. Mt. Vernon, NY. $470 \mathrm{pp}$.

Wickham, H. F., 1927. Supplement to Catalogue of North American Coleoptera described as fossils. pp. 53-56. Supplement 1919 to 1924 (inclusive) to Catalogue of the Coleoptera of America, north of Mexico. $78 \mathrm{pp}$. 
Wickham, H. F., 1933. Second supplement to Catalogue of North American Coleoptera described as fossils. pp. 103-105. In: Second and third supplements, 1925-1932 (inclusive) to Catalogue of the Coleoptera of America, north of Mexico. $112 \mathrm{pp}$.

Wilcox, J. A., 1965. A synopsis of North American Galerucinae (Coleoptera:Chrysomelidae). Bull. 400. New York State Mus. Sci. Serv. 226 pp.

Wood, D. N., Hardy, J. E. \& Harvey, A. P., 1989. Information sources in the earth sciences. Second Edition. Bowker-Saur. London. 518 pp.

Wollaston, T. V., 1863. Note on the remains of Coleoptera from the peat of Lexten brick-pit. Quart. Jour. Geol. Soc. London. 19, i:400 (no. 2), 401 (no. 9).

Wootton, R. J., 1981. Paleozoic Insects. Ann. Rev. Entomol. 26:319-344.
Zaddach, G., 1868. IV. Amber; its origin and history, as illustrated by the geology of Samland. Quart. Jour. Sci. 5:167-185.

Zeuner, F. E., 1962. Fossil insects from the Lower Lias of Charmouth, Dorset. Bulletin of the British Museum (Natural History) Geology (London). 7:153-171.

Zhang, J. F., 1989. Fossil insects from Shanwang, Shandong, China. Shandong Science and Technology Publishing House. Jinan, People's Republic of Chind. 459 pp. (In Chinese with English summary)

Zherikhin, V. V., 1970. Zoogeograficheskie svyazi paleogenovykh nasekomykh. Doklady na dvadtsat vtopom ye zhegodnom chtenii pamyati N. A. Kholodkovskogo. Akademiya Nauk SSSR, Vcesoyuzhoe entomologicheskoe obshchestvo. Izdatelistvo 'Nauka'. Leningrad. USSR. pp. 29-88. (In Cyrilic). 